T/61919se

OAK RIDGE

NATIONAL

LABORATORY

MARTIN MARIETTAX

\title{
HEAT-FLUX GAGE \\ THERMOPHOSPHOR SYSTEM
}

Software/Hardware Guide

Version 1.1

K. W. Tobin

August 1991

\section{MANAGED BY}

MARTIN MARIETTA ENERGY SYSTEMS, INC.

FOR THE UNITED STATES

DEPARTMENT OF ENERGY 
This report has been reproduced directly from the best available copy.

Avaliable to DOE and DOE contractors from the Office of Scientific and Tecthical information, P.O. Box 62. Oak Ridoe, TN 37831: prices available from (615) 576-8401. FTS 626-8401.

This report wee prepared as an account of work eponsored by an agency of the United States Government. Neither the Unitad States Government nor any aponcy thereot, nor any of their employees, makes any warrenty, express or implied, or assumes any logal liability or responeibility for the accuracy, complotenese, or usetuineses of any information, apparatus. product. or process disclosed, or represents that its use would not intringe privately owned rights. Reference herein to any specific commercial product. proceses, or service by trade name. trademank, manufacturer, or otherwise, does not necessarily constittute or imply its endorsemem, recommendation, or favoring by the United States Govermment or any egency thereof. The views and opinions of authors expressed herein do not necessarity state or reflect tinose of the United States Government or any apency thereof. 
Applied Technology Division

\title{
HEAT-FLUX GAGE THERMOPHOSPHOR SYSTEM \\ Software/Hardware Guide \\ Version 1.1
}

K. W. Tobin

Date Published-August 1991

\author{
Prepared by the \\ Oak Ridge National Laboratory \\ Oak Ridge, Tennessee 37831-7280 \\ managed by \\ MARTIN MARIETTA ENERGY SYSTEMS, INC. \\ for the \\ U.S. DEPARTMENT OF ENERGY \\ under contract DE-AC05-84OR21400
}




\section{CONTENTS}

LIST OF FIGURES $\ldots \ldots \ldots \ldots \ldots \ldots \ldots \ldots \ldots \ldots \ldots \ldots \ldots \ldots \ldots$

LIST OF SCREENS $\ldots \ldots \ldots \ldots \ldots \ldots \ldots \ldots \ldots \ldots \ldots \ldots \ldots \ldots \ldots$

ABSTRACT $\ldots \ldots \ldots \ldots \ldots \ldots \ldots \ldots \ldots \ldots \ldots \ldots \ldots \ldots \ldots \ldots \ldots \ldots \ldots \ldots$

1. INTRODUCTION $\ldots \ldots \ldots \ldots \ldots \ldots \ldots \ldots \ldots \ldots \ldots \ldots \ldots \ldots \ldots \ldots$

2. SYSTEM REQUIREMENTS $\ldots \ldots \ldots \ldots \ldots \ldots \ldots \ldots \ldots \ldots \ldots \ldots \ldots$

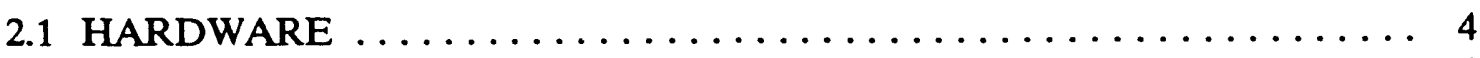

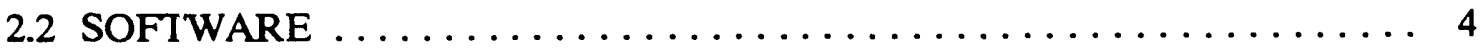

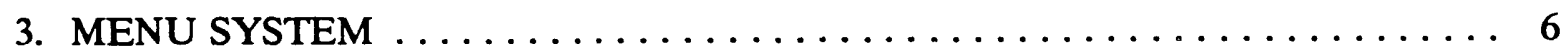

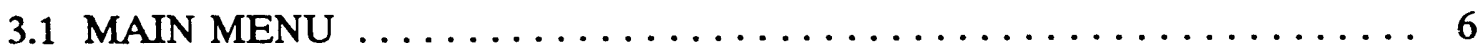

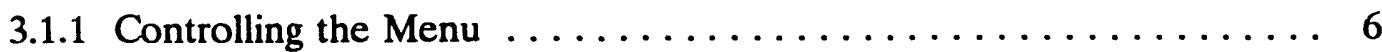

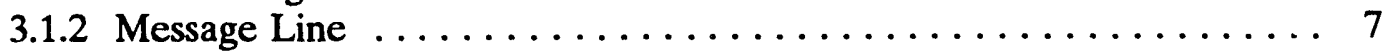

3.1.3 Status Box ...................... 7

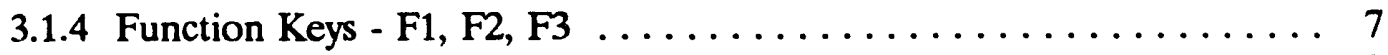

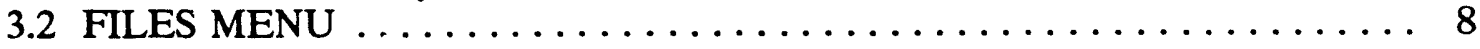

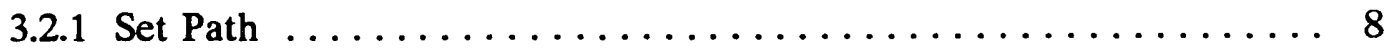

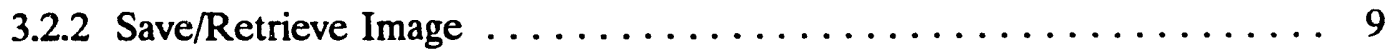

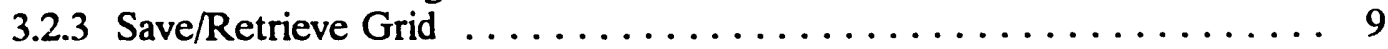

3.2.4 Save/Retrieve Configuration $\ldots \ldots \ldots \ldots \ldots \ldots \ldots \ldots \ldots \ldots \ldots \ldots \ldots$

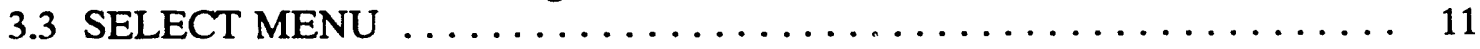

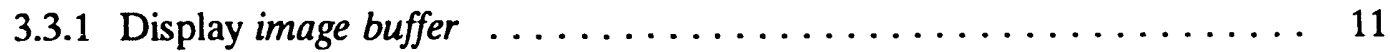

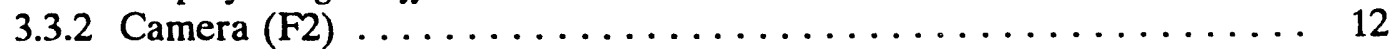

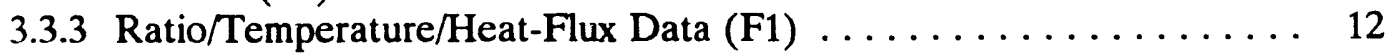

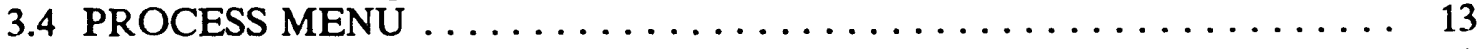

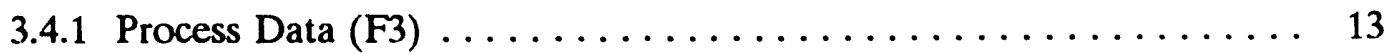

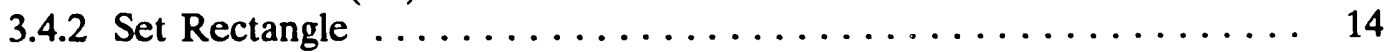

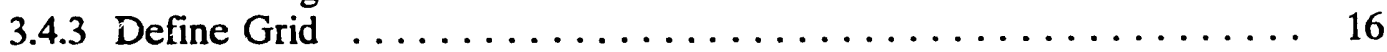

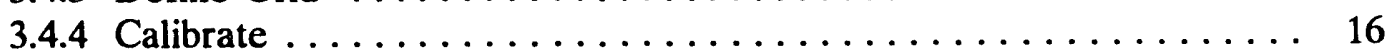

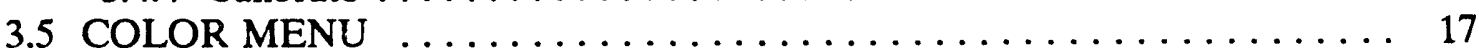

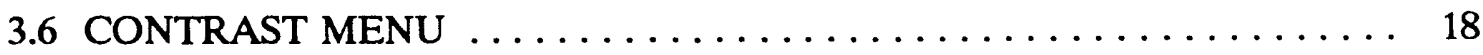

3.7 PROGRAM EXIT . . . . . . . . . . . . . . . . . . 19

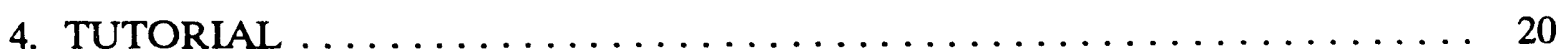

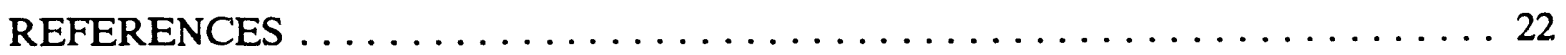




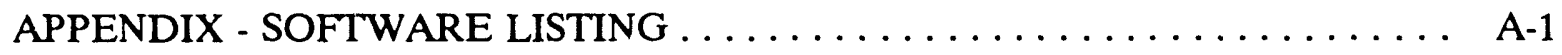

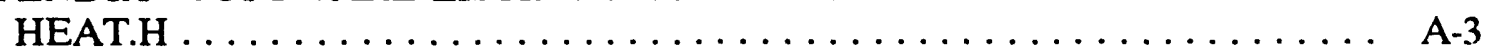

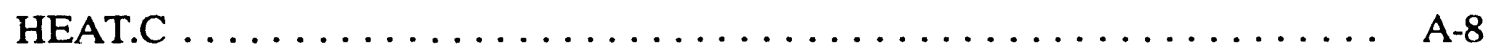

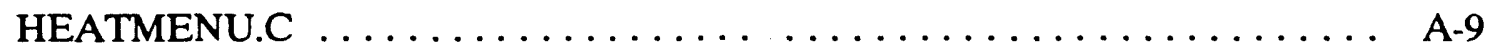

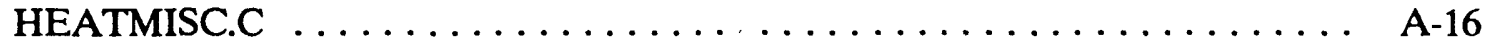

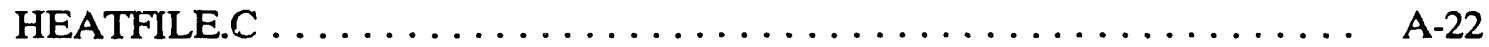

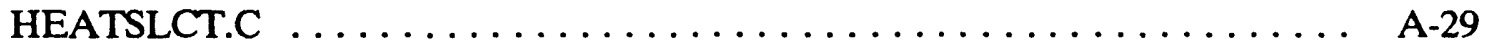

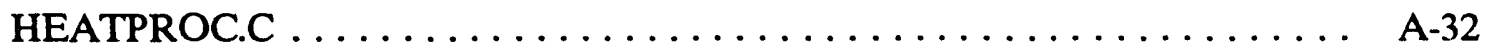

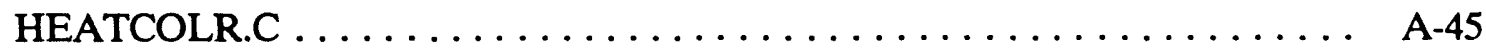

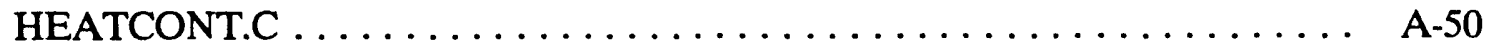




\section{LIST OF FIGURES}

1. Geometry associated with the $\mathrm{Gd}_{2} \mathrm{O}_{2} \mathrm{~S}$ :Tb heat-flux gage $\ldots \ldots \ldots \ldots \ldots \ldots \ldots$

2. Schematic representation of the heat-flux measurement system utilizing the DT-2861 image processing hardware $\ldots \ldots \ldots \ldots \ldots \ldots \ldots \ldots \ldots \ldots$

3. Representation of the gage geometry and software cursor locations used with the Set Rectangle and Define Grid commands

\section{LIST OF SCREENS}

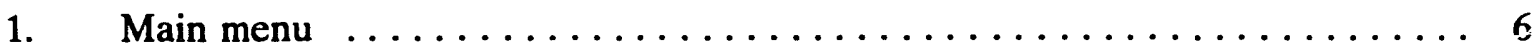

2. Files menu $\ldots \ldots \ldots \ldots \ldots \ldots \ldots \ldots \ldots \ldots \ldots \ldots \ldots \ldots \ldots \ldots \ldots \ldots$

3. Files menu showing the Set Path option $\ldots \ldots \ldots \ldots \ldots \ldots \ldots \ldots \ldots$

4. Files menu showing the Save/Retrieve Image option $\ldots \ldots \ldots \ldots \ldots \ldots \ldots$

5. Files menu showing the Save/Retrieve Grid option $\ldots \ldots \ldots \ldots \ldots \ldots$

6. Files menu showing the Save/Retrieve Configuration option $\ldots \ldots \ldots \ldots \ldots 11$

7. Select menu options $\ldots \ldots \ldots \ldots \ldots \ldots \ldots \ldots \ldots \ldots \ldots \ldots \ldots \ldots \ldots \ldots \ldots$

8. Process data menu option $\ldots \ldots \ldots \ldots \ldots \ldots \ldots \ldots \ldots \ldots \ldots \ldots \ldots$

9. Example output file (*.SUM) generated using the Process Data function . . . 14

10. Calibrate submenu accessed through the PROCESS/Calibrate option . . . . . 17

11. Color menu option $\ldots \ldots \ldots \ldots \ldots \ldots \ldots \ldots \ldots \ldots \ldots \ldots \ldots \ldots$

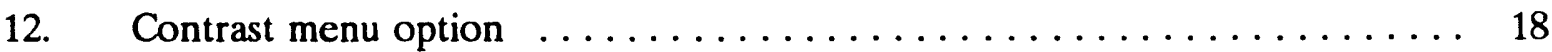

13. User is prompted to verify exit $\ldots \ldots \ldots \ldots \ldots \ldots \ldots \ldots \ldots \ldots \ldots$

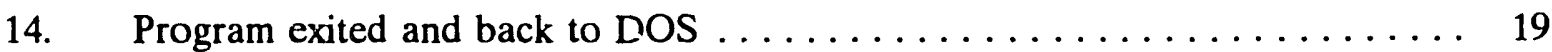




\begin{abstract}
This document describes the installation, hardware requirements, and application of the Heat-Flux Gage (Version 1.0) software package developed by the Oak Ridge National Laboratory, Applied Technology Division. The developed software is a single component of a thermographic phosphor-based temperature and heat-flux measurement system. The heatflux transducer was developed by EG\&G Energy Measurements Systems and consists of a 1-by 1-in. polymethylpentene sheet coated on the front and back with a repeating thermographic phosphor pattern. The phosphor chosen for this application is gadolinium oxysulphide doped with terbium. This compound has a sensitive temperature response from 10 to $65.6^{\circ} \mathrm{C}\left(50-150^{\circ} \mathrm{F}\right)$ for the $415-$ and $490-\mathrm{nm}$ spectral emission lines.
\end{abstract}




\section{INTRODUCTION}

The Heat-Flux Gage software package, Version 1.0, has been designed by the Oak Ridge National Laboratory, Applied Technology Division (ORNL/ATD) for use with the twodimensional heat-flux gage, which was concurrently developed by EG\&G Energy Measurement Systems, Santa Barbara, California.

The heat-flux gage consists of a $25-\mu \mathrm{m}$ thick, 1- by 1-in. sheet of polymethylpentene (PMP). A gadolinium oxysulphide thermographic phosphor doped with terbium $\left(\mathrm{Gd}_{2} \mathrm{O}_{2} \mathrm{~S}: \mathrm{Tb}\right)$ has been deposited on the front and back surface of the PMP in a regular triangular array. When viewed through the surface, the front and back phosphor coatings correspond to unit rectangular regions (see Fig. 1) that the software utilizes to extract the two-dimensional temparature and heat flux.

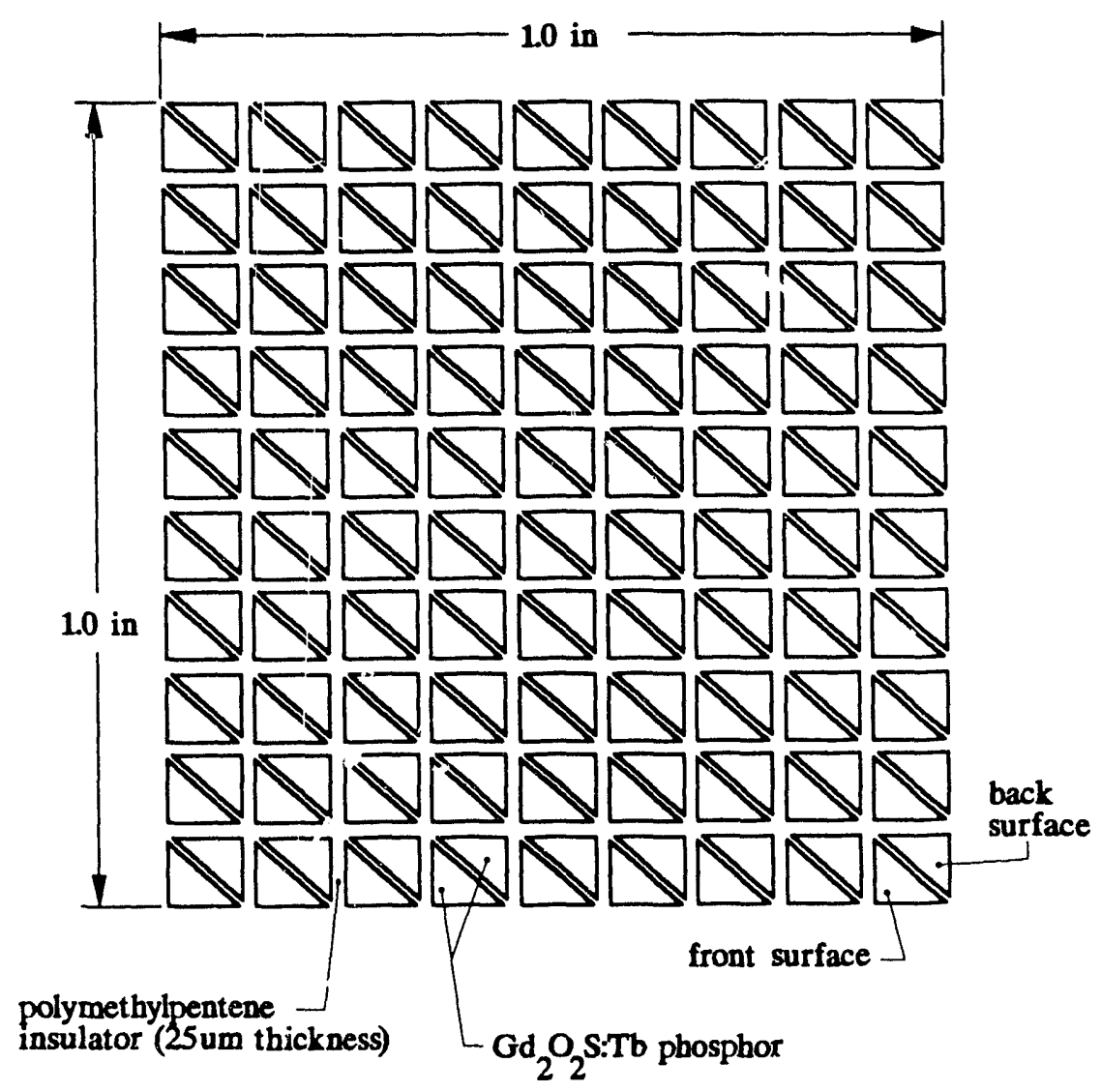

Fig. 1. Geometry associated with the $\mathrm{Gd}_{2} \mathrm{O}_{2} \mathrm{~S}: 1 \mathrm{~b}$ heat-flux gage.

The temperature information on the front and back surfaces is obtained by the ratio of the 415- and 490-nm spectral lines of the phosphor emission when illuminated with a steady-state ultraviolet light source. Since a ratio is incorporated to generate the required temperature information, the following nomenclature will be used throughout this document: 
NUMERATOR

NUMERATOR BACKGROUND

DENOMINATOR

DENOMINATOR BACKGROUND
- numerator image of the ratio, $n(x, y)$

- numerator background (no ultraviolet source), $n_{b}(x, y)$

- denominator image of the ratio, $d(x, y)$

- denominator background image of the ratio, $d_{b}(x, y)$

The ratio image data are calibrated to temperature by the following relationship,

$$
T(x, y)=f\left[\frac{n(x, y)-n_{b}(x, y)}{d(x, y)-d_{b}(x, y)}\right]=f[r(x, y)],
$$

where $r(x, y)$ is the corrected image ratio and $f[r(x, y)]$ has been shown to be the linear function,

$$
T(x, y)=f[r(x, y)]=T_{h}-\left(T_{h}-T_{p}\right)\left(\frac{r-r_{l}}{r_{h}-r_{l}}\right),
$$

for the $\mathrm{Gd}_{2} \mathrm{O}_{2} \mathrm{~S}: \mathrm{Tb}$ compound over the temperature range from $1 \mathrm{C}$ to $65.6^{\circ} \mathrm{C}\left(50-150^{\circ} \mathrm{F}\right){ }^{1,2}$ The constants, $T_{h}, T_{l}, r_{h}$, and $r_{l}$, are calibration constants for the phosphor compound.

The calibrated amplitude-based ratio is used to generate the temperature of the associated surface. The surface heat flux estimate is then made by application of Fourier's law of conductic ${ }^{3}{ }^{3}$

$$
q^{\prime \prime}(x, y, z)=-k \nabla T(x, y, z),
$$

where $q^{\prime \prime}(x, y, z)$ is the heat flux, $k$ is the thermoconductivity of the material, and $T(x, y, z)$ is the temperature. For the current application it is assumed that the PMP is sufficiently thin to make the approximation that the heat flux, $q^{\prime \prime}$, can be expressed as,

$$
q^{\prime \prime}(x, y)=-k \frac{\Delta T(x, y)}{\Delta z}
$$

or, for the gage design shown in Fig. 1,

$$
q^{\prime \prime}(x, y) \propto-k \frac{T(x, y)_{\text {front }}-T(x, y)_{b a c k}}{\Delta z_{P M P}}
$$


where $T(x, y)_{\text {front }}$ and $T(x, y)_{\text {back }}$ are the surface temperatures at unit cell $(x, y)$ on the front and back of the gage, respectively, and $\Delta z_{P M P}$ is the thickness of the PMP material.

The heat flux is determined in the code by combining Eqs.(2) and (5) to give,

$$
q^{\prime \prime}(x, y)=-\frac{k}{\Delta z_{P M P}}\left(T_{h}-T_{l}\right)\left(\frac{r_{\text {back }}(x, y)-r_{\text {front }}(x, y)}{r_{h}-r_{l}}\right) .
$$

The heat-flux gage will be mounted on a fixed surface in a wind tunnel, as shown in Fig. 2 , and viewed with an intensified $C C D$ video camera. The ultraviolet illumination source is required to be steady state for this application because of constraints of the imaging hardware, which require operation at standard RS-170 video rates (30 frames/s).

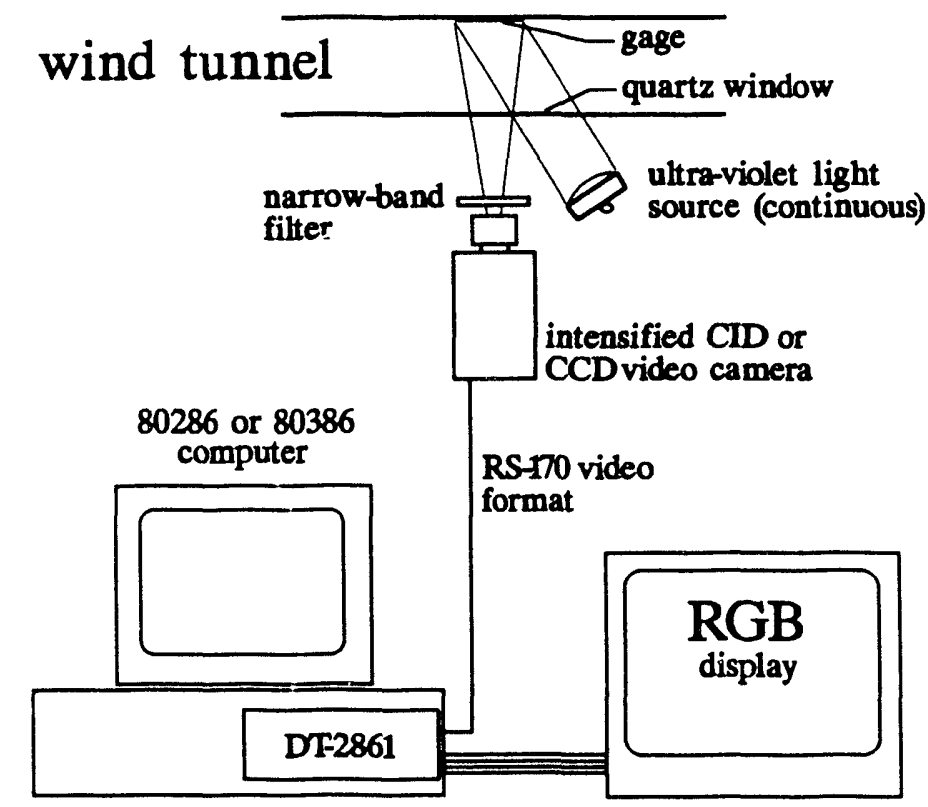

Fig. 2 Schematic representation of the heat-flux measurement system utilizing the DT-2861 image processing hardware.

The camera will image the gage surface through a quartz window on the wind tunnel and through one of the two narrow-band optical filters (i.e., $415 \mathrm{~nm}$ or $490 \mathrm{~nm}$ ). Currently, development, these filters must be positioned by hand. The imaged information is exported by the camera in analog RS-170 video format where it is subsequently digitized by a DT-2861 Data Translation ${ }^{\mathrm{TM}}$ image processing board mounted in an 80386 or 80286 computer system. The captured and processed images are displayed on an RGB monitor for viewing and verification of the experiment setup. The resultant temperature and heat-flux data are also displayed and stor' $\mathrm{d}$ in a data file for postprocessing and analysis. 


\section{SYSTEM REQUIREMENTS}

\subsection{HARDWARE}

The heat-flux gage system was developed for operation on an 80386 or 80286 computer with or without a math co-processor, using DOS 3.2 or higher. The program works in conjunction with a DT-2861 Data Translation ${ }^{\mathrm{TM}}$ image processing module. The DT-2861 is a full-sized plug-in card that requires a 16-bit AT-style slot. The processed images are displayed on a standard color monitor that has the following four inputs:

\section{Red (video in) \\ Green (video in) \\ Blue (video in) \\ External Sync}

These video lines are located on the $\mathrm{BNC}$ ribhon cable, which must be purchased with the DT-2861 card. The following hardware and software defines the prototype heat-flux gage system:

\section{Data Translation:}

DT-2861
EP197
IDRV61.SYS
ISCLLIB.LIB
ISDEFS.H
ISERRS.H

Microsoft:

MS-DOS

C-compiler

- version 3.2 (or higher)

- version 5.0 (or higher)

- 60-Hz frame grabber

- coaxial cable assembly

- software driver

- SP0215 definition file

\section{Computer:}

- SP0215 C-binding library support

- SP0215 error definition file

80286 or 80386 system

80287 or 80387 co-processor (optional)

\section{SOFTWARE}

The software control program was developed using the Microsoft C-compiler (version 5.1) and the Vitamin-C screen $I / O$ library (version 3.1a), along with the SP0215 library associated with the image processing hardware. The following lists the source code files and sizes developed to implement the heat-flux gage algorithm: 
HEAT

HEAT.IMG

HEAT.PTH

HEAT.H

HEAT.C

HEATMENU.C

HEATMISC.C

HEATFILE.C

HEATSLCT.C

HEATPROC.C

HEATCOLR.C

HEATCONT.C
720

262656

22

10909

739

13173

9628

12475

3929

25246

7449

3012
- make file for compilation of the code

- default image screen

- start-up data path information

- definition (header) file for the C code

- main control module source code

- menu definition source code

- miscellaneous custom program tools

- disk I/O etc. source code

- DT-2861 buffer select etc. source code

- algorithm implementation source code

- false color, zoom/pan control, etc.

- contrast enhancement, etc.

An example set of system output files resulting from a test run is given here.

$\begin{array}{ll}\text { DEMO RD. } & 235 \\ \text { DEMO.SUM } & 1261 \\ \text { DEMO.DAT } & 513 \\ \text { DEMO.CON } & 106 \\ \text { DEMO.001 } & 262656 \\ \text { DEMO.002 } & 262656 \\ \text { DEMO.003 } & 262656 \\ \text { DEMO.004 } & 262656\end{array}$

- grid coordinate data

- full run summary

- short run summary

- configuration/calibration constants

- numerator background image

- numerator fluorescence image

- denominator background image

- denominator fluorescence image

The executable run file is as follows:

\section{HEAT.EXE}

176646

When the directory is selected where the executable file resides, the program is started by typing the following:

\section{C:L... $>$ HEAT}

If the DT-2861 is properly installed, the program will load normally with the following screen message and begin at the main system menu.

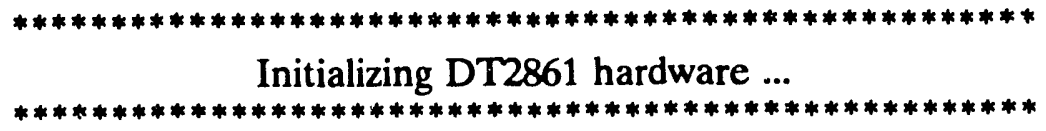

If the $\mathrm{Di}^{-}-2861$ is improperly installed or malfunctioning, then the program will automatically exit back to the DOS prompt at this point with the error message,

Missing or bad DT-2861 imaging hardware. 


\section{MENU SYSTEM}

\subsection{MAIN MENU}

When the program has been successfully loaded, the main control menu will be displayed on the computer monitor as shown in Screen 1.

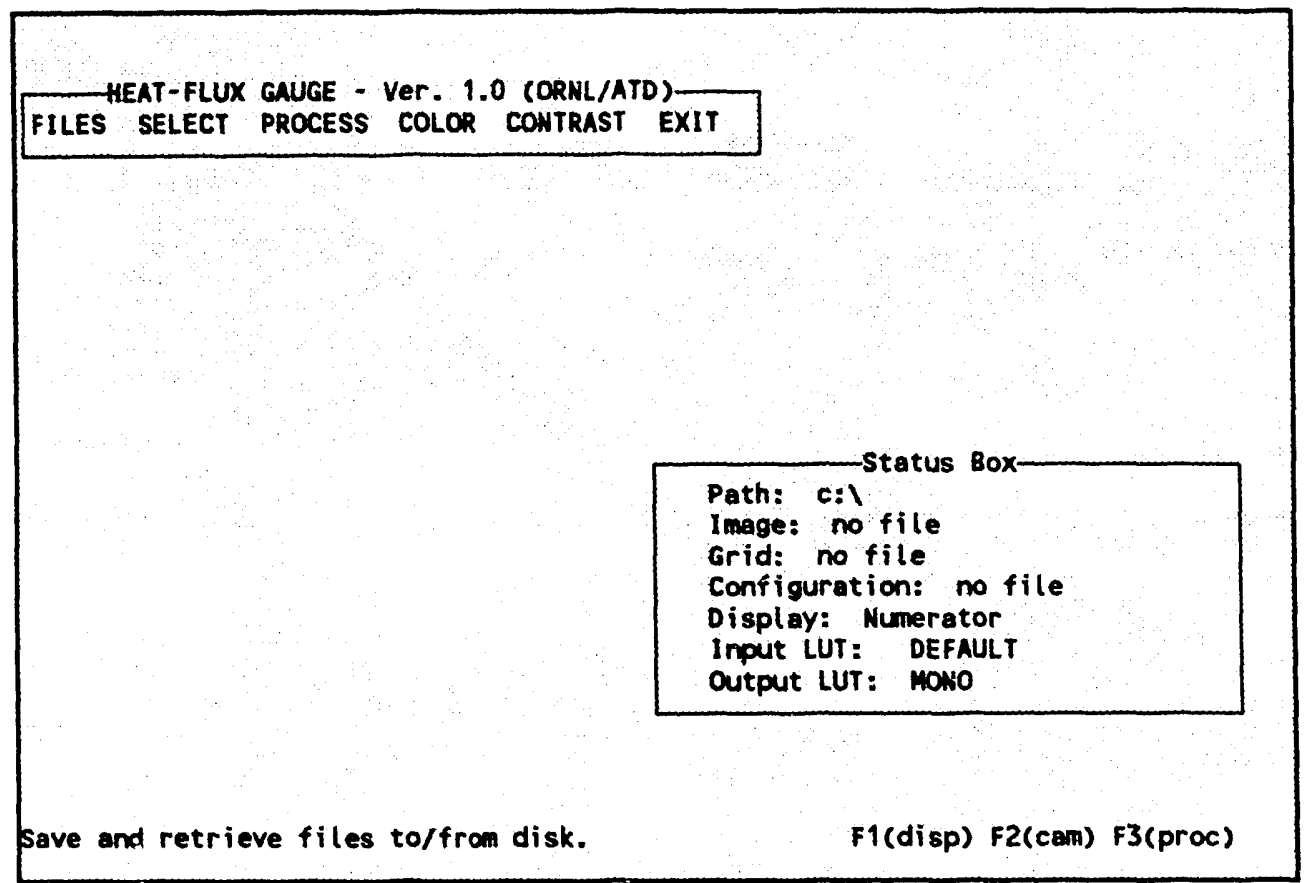

Screen 1. Main menu.

The main menu contains all necessary information to enable capturing, saving, retrieving, processing, and calibrating image data. The FILES menu invokes a submenu that allows data disk $\mathrm{I} / \mathrm{O}$ access and default path definitions. The SELECT menu also invokes a submenu that allows selection of required data buffers for storing and processing fluorescence image data. The PROCESS menu and submenu allow processing of the captured data, grid geometry definitions, and access to the system calibration tools. For the current version of the heat-flux software, the COLOR and CONTRAST menu options will be of little additional use. Their current capabilities and future purpose will be described later in this document.

\subsubsection{Controlling the Menu}

The menu system has been designed to allow easy access to any portion of the program such as nodifying the configuration, loading and saving data, or processing image data. The menu is controlled by the UP, DOWN, RIGHT, and LEFT arrow keys on the computer key pad. A highlighted menu item is selected by pressing the ENTER key. When the ENTER key is pressed, either a submenu will be invoked, the user will be prompted for input, or an operation will be performed on the image data. When a submenu or menu option has been invoked the user can always back up one level or terminate an option by using the ESC key. 


\subsubsection{Message Line}

At the bottom left corner of the menu screen a message line is continually displayed that contains a short definition of the current menu option. (e.g., Screen 1 displays "Save and retrieve files to/from disk.") This message is continually updated as the menu selector box traverses the screen. When a calculation is being performed on the image data, the message line will display information about the current process. When the process is complete, the message line will resume its previous function.

\subsubsection{Status Box}

The status box in the lower right corner of the display (see Screen 1) is continually updated to reflect the current state of the program. The status box parameters are defined as follows:

Path: current $\mathrm{I} / \mathrm{O}$ path for the storage of images, grid coordinates, process results, and system configuration data.

Image: displays the name of the most current image file read from the disk path. If no file has been retrieved from the disk, a no file status will be displayed.

Grid: name of the most current grid coordinate file read from the current path. If no grid has been selected or a new grid has been defined during the current session, a no file status will be displayed.

Configuration: name of the configuration file read from the current path. If no configuration has been selected, a no file status will be displayed.

Display: name of the image buffer which is currently being displayed on the RGB monitor (e.g., NUMERATOR, RATIO, HEAT-FLUX, etc.). When the program is initially started, the NUMERATOR image (initially blank) is displayed.

Input LUT (look-up table): defines the AND input gain for the DT-2861 system. The standard (DEFAULT) table is linear. This table can be mudified by using the CONTRAST option on the main menu but this tool has not been fully developed to date.

Output LUT (look-up table): defines the RGB color mapping on the RGB display device. This tool is accessed using the COLOR menu option. While this tool has not been fully developed to date, its use will not adversely affect the processing of image data.

\subsubsection{Function Keys - F1, F2, F3}

Currently, three function keys are defined to allow easier access to some of the frequently used menu options. The definitions of these keys are permanently displayed in the lower right-hand corner of the screen. The F1 key toggles through the resultant image(s) display on the RGB monitor. When a data set has been processed, the ratio, temperature, and heat flux can be chosen for display. The F2 key will turn on the camera and allow the 
collection of data into the current active buffer (as displayed in the status box). The F3 key will invoke the process routine for the image data set, the result of which is the ratio, temperature, and heat-flux distribution on the gage surface. All of these function keys reduce the number of key strokes necessary to execute the indicated command. All of the commands executed by a function key are otherwise accessed through the menu system.

\subsection{FILES MENU}

The FILES menu options are shown in Screen 2. This submenu allows for a change in default data path, image storage and retrieval, grid coordinate storage and retrieval, and system configuration storage and retrieval.

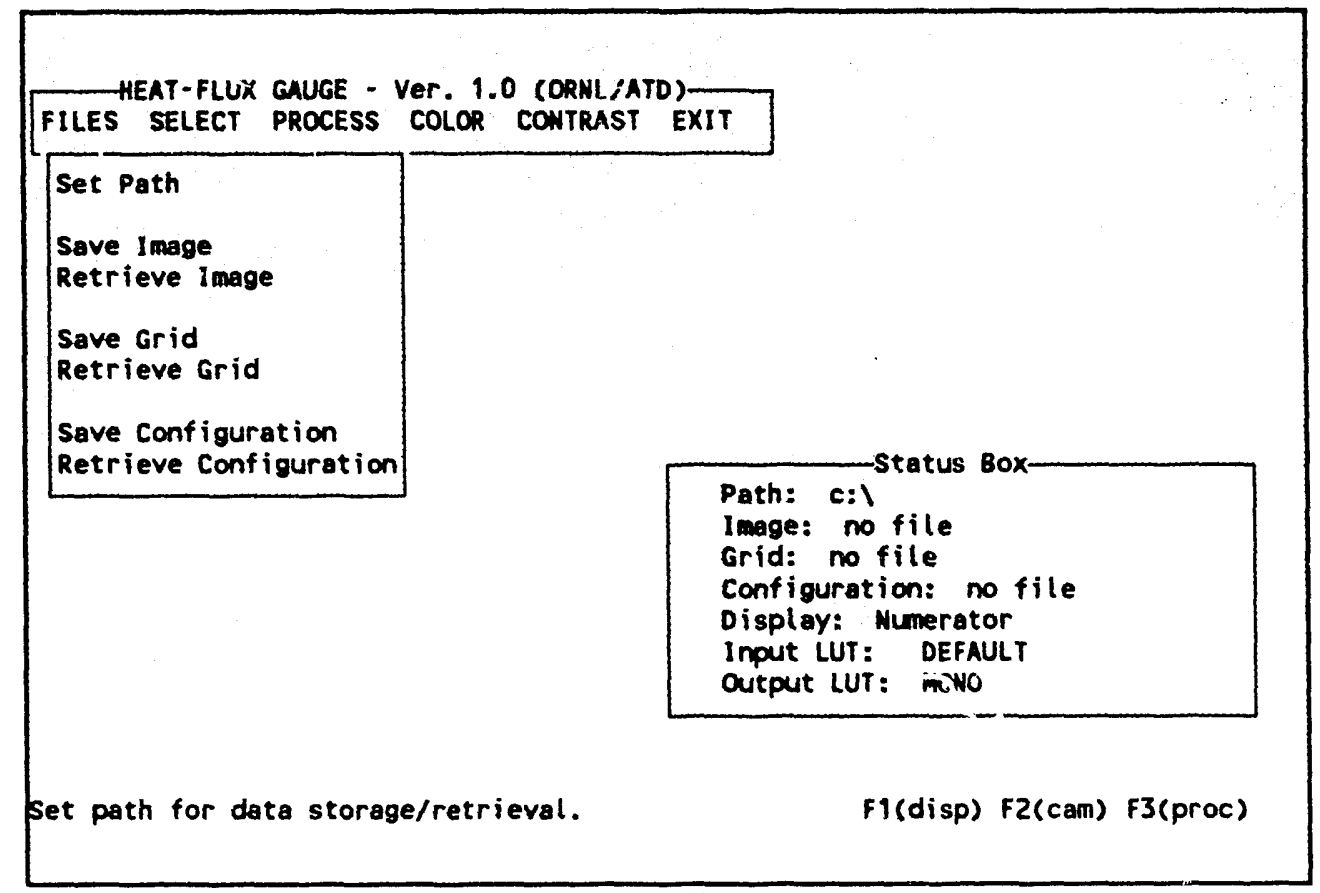

Screen 2 Files menu.

\subsection{Set Path}

The Set Path option (see Screen 3) allows the user to define the data storage area for the computer I/O. All image data, grid coordinate data, and configuration data will be written to this directory. Also, when the process function is invoked, the user will be prompted for a file name under which to store the long and abbreviated data summary. This data will also be located in the current path. If the user wishes to utilize several subdirectories for storing program data, the Set Path option can be modified at any time during the program execution. When the program exits, the current path will be the default start path the next time the program is executed. 


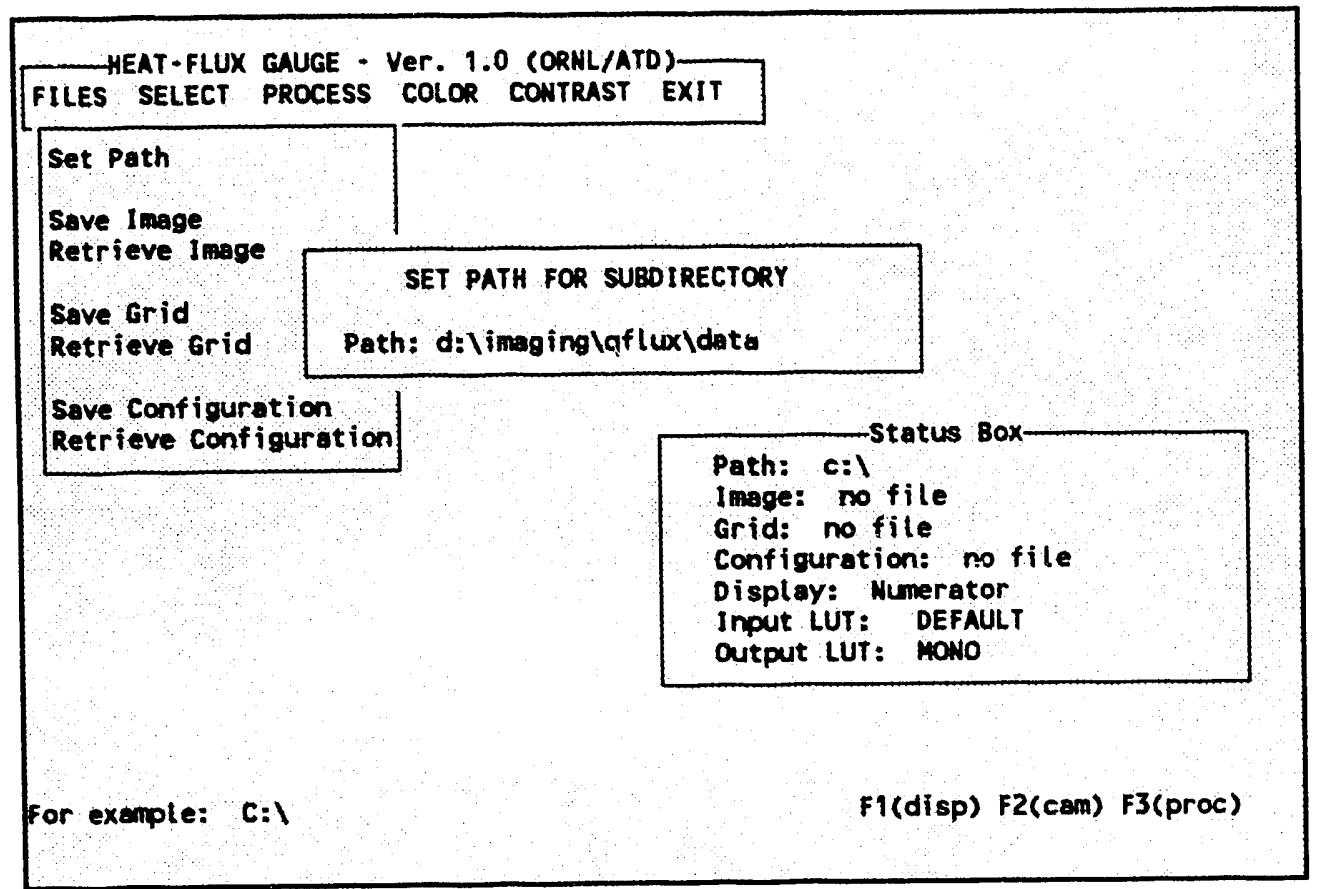

Screen 3. Files menu showing the Set Path option.

\subsubsection{Save/Retrieve Image}

The Save/Retrieve Image option is shown on Screen 4. The four data buffersNUMERATOR, NUMERATOR BACKGROUND, DENOMINATOR, and DENOMINATOR BACKGROUND - must be maintained on disk if the data are to be reanalyzed at a later time (e.g., if a modified calibration is to be employed, etc.). When the user is prompted for a file name (see Screen 4) four files will be created sharing this name.

$$
\begin{aligned}
& <\text { file name }>.001 \\
& <\text { file name }>.002 \\
& <\text { file name }>.003 \\
& <\text { file name }>.004
\end{aligned}
$$

- numerator background image

- numerator fluorescence image

- denominator background image

- denominator fluorescence image

It is important to note that only one name (no file type) should be entered. This method of naming a data set ensures that data from separate runs are kept together for future reference.

\subsection{Save/Retrieve Grid}

The Save/Retrieve Grid option is shown on Screen 5. The image grid is a set of coordinates defined by the user under the PROCESS/Define Grid submenu option. The grid file contains the $(x, y)$ coordinates of the heat-flux unit cell for future use by the program. This option allows several runs to occur without redefining the gage geometry and unit cell location. The current hardware (DT-2861) employed to process the gage data has no built-in morphological processes or functions; thus, automatic detection of the unit cell location is a manual task for the user. When a grid has been manually defined, it can be stored and recalled for use at a later date as long as the experiment geometry remains constant. If the 
geometry changes, a new grid must be manually generated. The filetype *.GRD is automatically appended to the user defined file name.

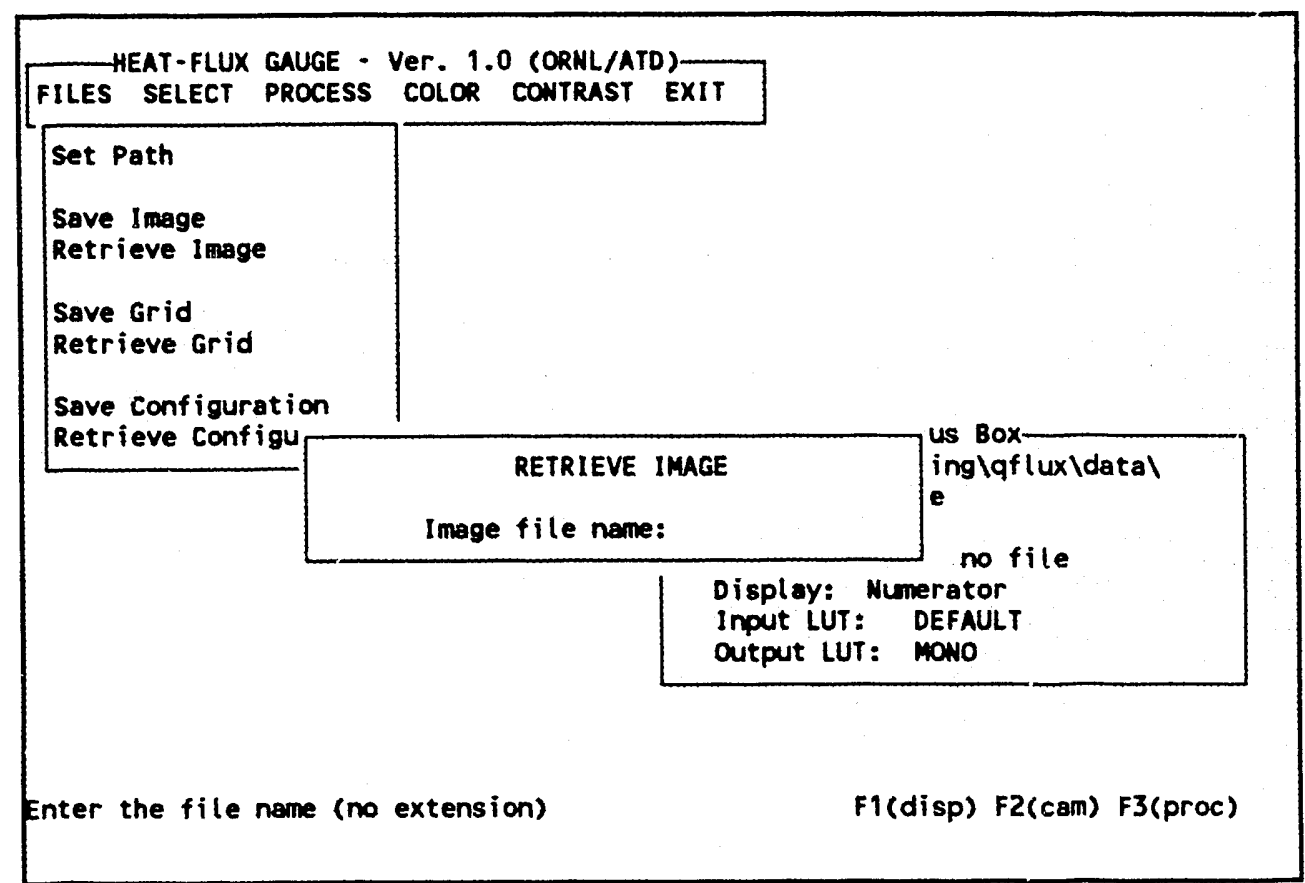

Screen 4. Files menu showing the Save/Retrieve Image option.

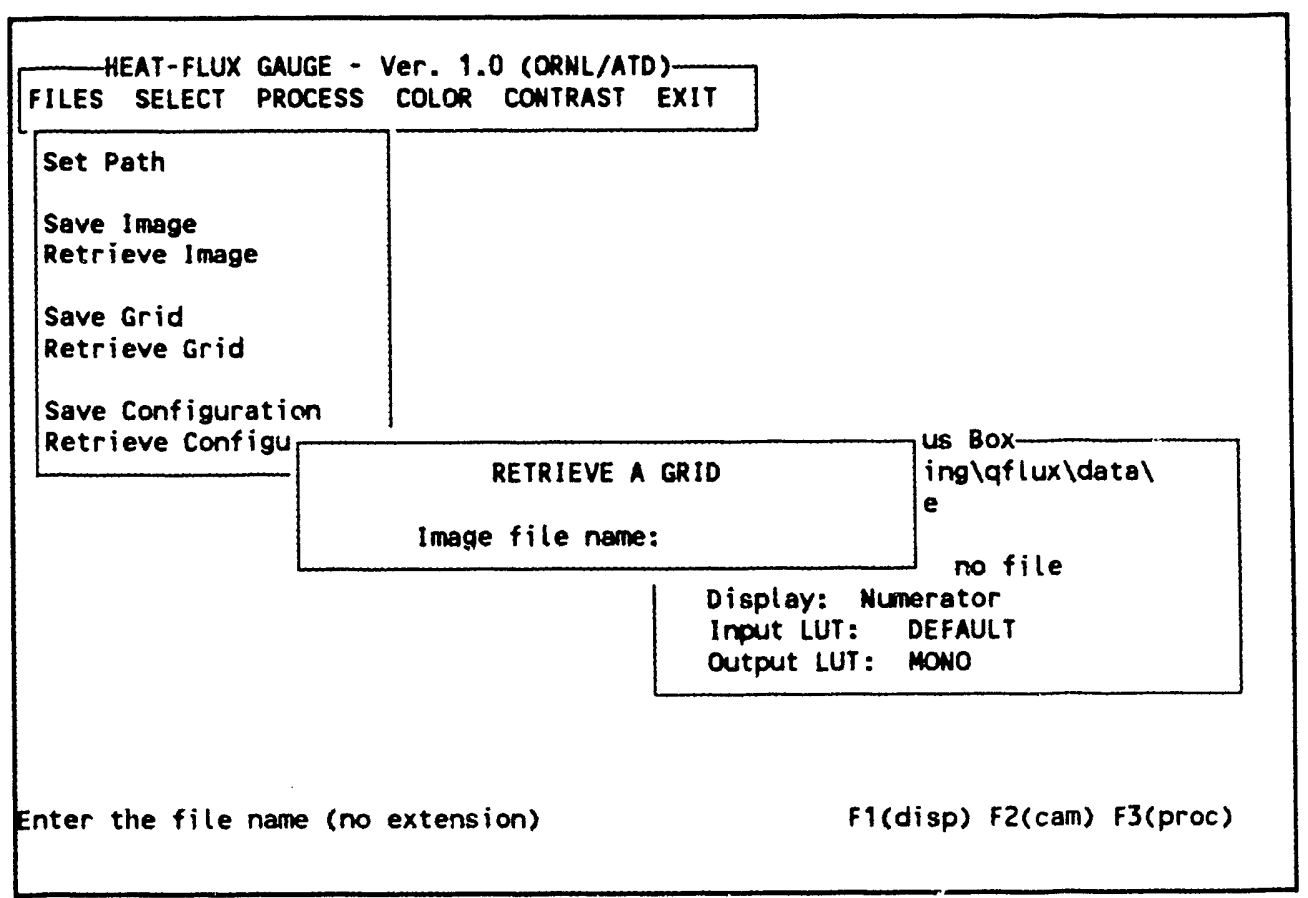

Screen 5. Files menu showing the Save/Retrieve Grid option. 


\subsubsection{Save/Retrieve Configuration}

The Save/Retrieve Configuration option is shown on Screen 6. The system configuration file contains the current calibration data for the thermographic phosphor being used. These items include the phosphor ratio levels, $r_{h}, r_{l}$; high and low temperature limits, $T_{h}, T_{l}$, on the calibration; thermal conductivity of the PMP, $k$; and the PMP thickness, $\Delta z_{P M P}$. These data are updated under the PROCESS/Calibrate submenu. There is also information related to the dimensions and geometry of the heat-flux gage itself. These data are updated under the PROCESS/Set Rectangle submenu. The filetype *.CFG is automatically appended to the user defined file name. Several sets of configuration can be independently applied to a given set of image data if desired.

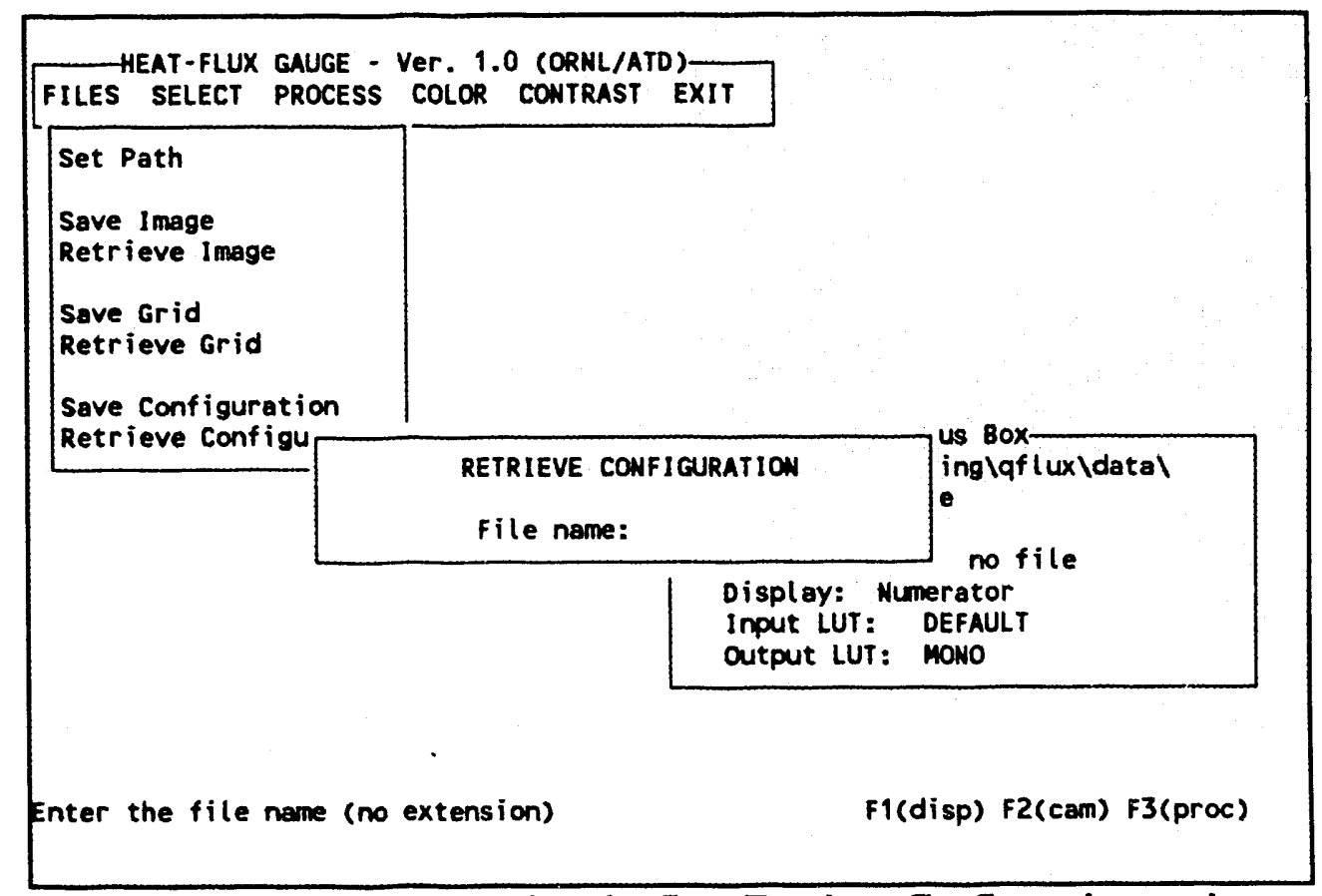

Screen 6. Files menu showing the Save/Retrieve Configuration option.

\subsection{SELECT MENU}

The SELECT menu, shown on Screen 7, allows for input of image data to the DT-2861 frame buffer system. The image buffers must be filed with the proper phosphor fluorescence information before the PROCESS menu or F3(proc) key can be used. There are two ways to load data into the image buffers. Data can be loaded from the computer disk drive as described above, or it can be captured from the intensified video device.

\subsubsection{Display image buffer}

The Display Numerator/Denominator/... options on the SELECT submenu are used to update the RGB display to the selected image and to set the active buffer status (shown in the status box). An active buffer is the only DT-2861 memory location that will be accessed when the video source is activated. Therefore, to load the NUMERATOR data buffer with 
the properly filtered (e.g., $415 \mathrm{~nm}$ ) fluorescence data, the Display Numerator option must be selected on the submenu prior to activating the video device. The same is true for all subsequent image buffers.

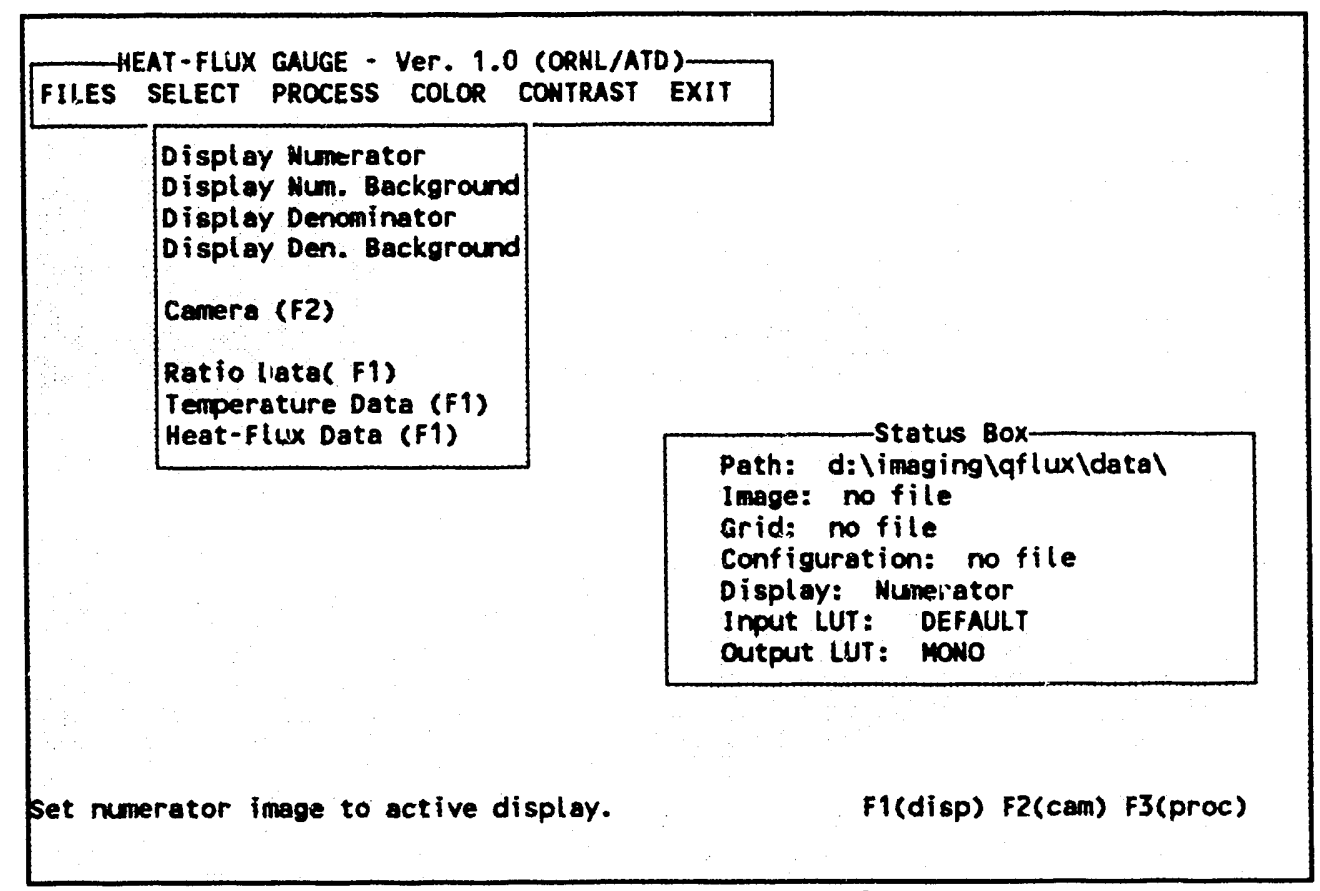

Screen 7. Select menu options.

\subsubsection{Camera (F2)}

The Camera option activates the video device and allows the data buffer to be filled with the appropriate image data. The same result is achieved if the F2 key is depressed. When activated, the system sets the synchronization (sync) source to external thus allowing the camera to run free until another key hit is registered. This allows for image pass through (live image play) and inspection while the experiment is being set up. Note that when a single data buffer is filled, no more adjustments to the experiment geometry should be made. Even slight movement of the experiment components can throw off the predefined grid coordinates and image correlations, thus requiring a complete new configuration. When a key hit is registered, the system is set back to internal sync drive and the camera can be turned off or removed from the system input.

\subsubsection{Ratio/Temperature/Heat-Flux Data (F1)}

The Ratio/Temperature/Heat-Flux Data options allow selection of these image buffers for redisplay when the data has been processed. The F1 key can be used to step through these buffers from any location in the menu system. The Ratio Data buffer displays the numerical calculation per unit cell of the emission ratio on the front and back side of the heat-flux gage. The Temperature Data buffer displays the corresponding calculated temperatures at each unit cell location [based on Eq.(2)], and the Heat-Flux Data buffer shows the corresponding heat-flux estimate [based on Eq.(6)]. 


\subsection{PROCESS MENU}

The PROCESS menu, shown in Screen 8, performs all program processing and calibration tasks. It should be invoked only after the data buffers (SELECT menu options, etc.) have been properly loaded.

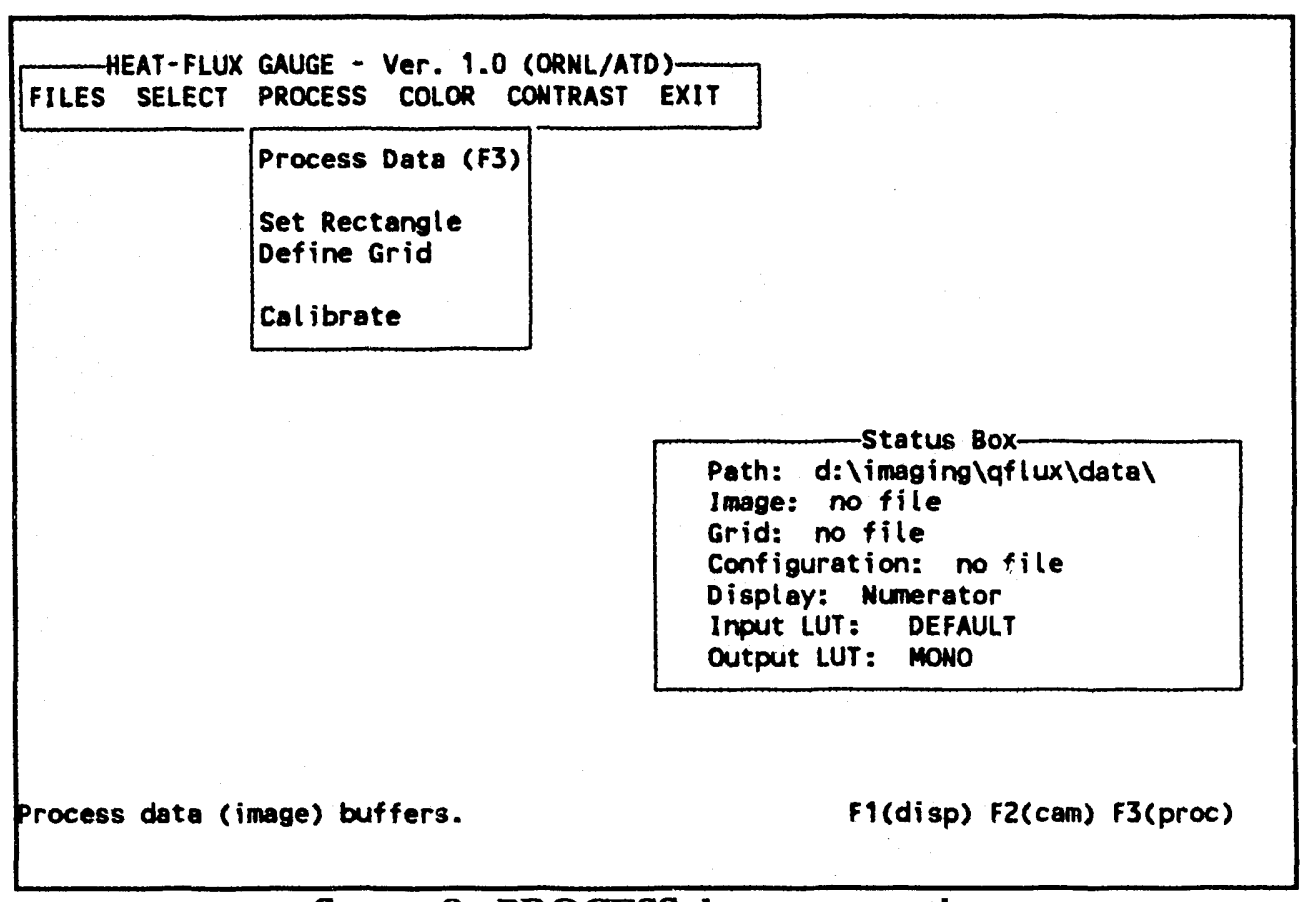

Screen 8. PROCESS data menu option.

\subsubsection{Process Data (F3)}

The Process Data option performs the point-wise calculation of temperature and surface heat flux on the basis of Eqs.(2) and (6). To perform this process, it is assumed that the NUMERATOR data buffers contain the 415-nm spectral information, while the DENOMINATOR buffers contain the 490-nm spectral information. The user should ensure that this requirement is met. There is no programmatic verification. When the data has been processed, the results are displayed on the RGB monitor and can be accessed through the SELECT menu or by using the F1 function key. Also, the user will be prompted for a file name under which to save a disk version summary and data listing ( ${ }^{*}$.SUM and ${ }^{*}$.DAT). The data files will be located in the directory specified by the current path (as displayed in the Status Box). Screen 9 shows an example summary file ( $\left.{ }^{*} . S U M\right)$, which can be accessed after leaving the HEAT.EXE program.

The first line of the summary file shows the current program version. The next four lines show the status of the system when the data was generated (i.e., the current path and the list of image, grid, and calibration files that were being used). The next five lines contain the current calibration parameters that were contained in the configuration file. The remaining data are a list of $(x, y)$ coordinates, ratio, temperature, and heat-flux data. The *.DAT file contains the $(x, y)$ coordinate data, the temperature data, and the heat-flux data, 
respectively. This file has been generated to allow easy access to code data by second party graphing and data analysis software packages. The summary and data output files are stored in ASCII format and can be edited or viewed with any commercial text editor.

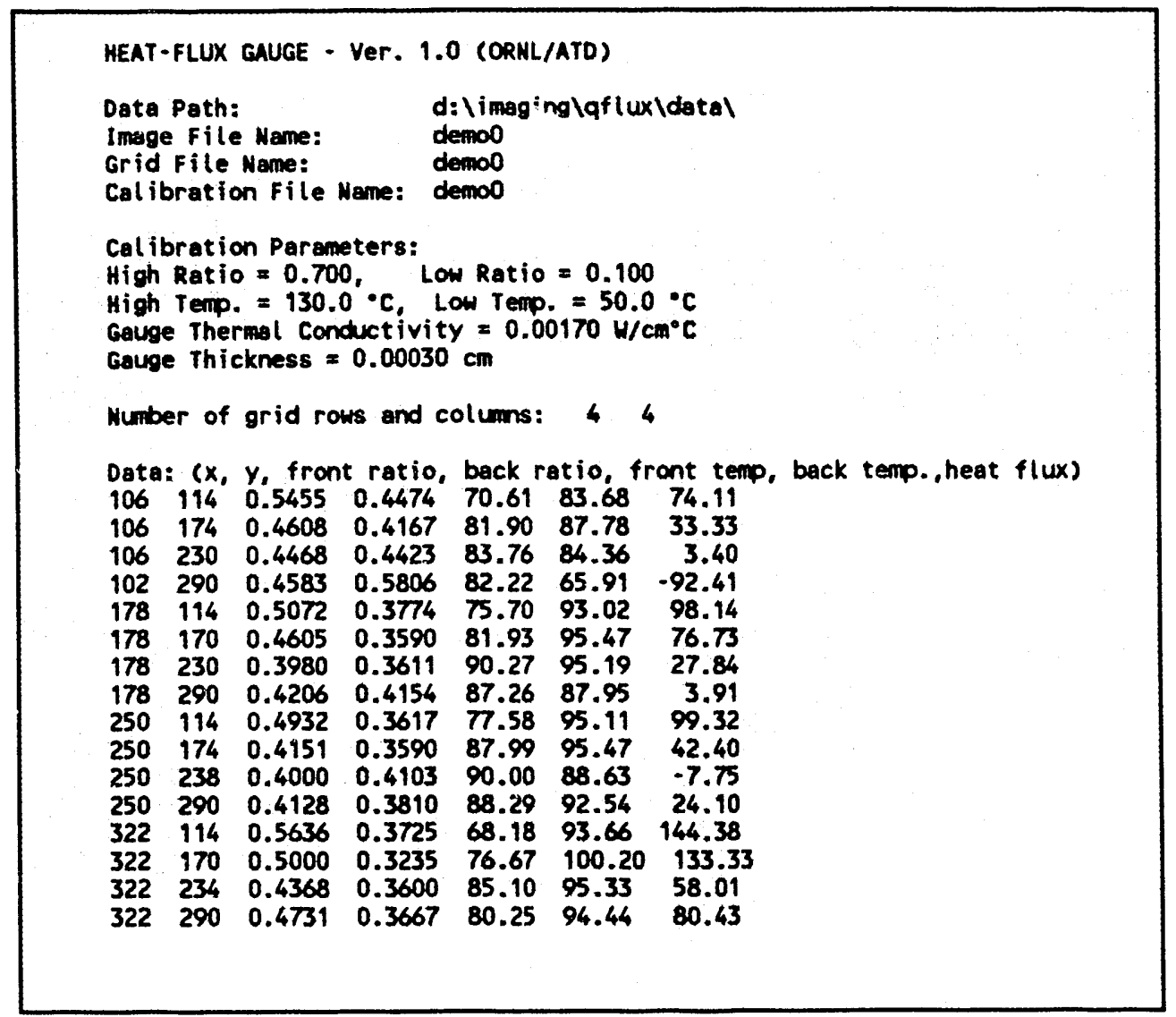

Screen 9. Example output file (*.SUM) generated using the Process Data function.

\subsubsection{Set Rectangle}

The Set Rectangle option configures the heat-flux gage for the current experiment geometry. This option must be used when initially configuring the system. The resultant measurement data are maintained in the configuration file when the Store Configuration option is selected (FILES menu).

When activated, a window will open on the computer screen querying the user for a data window size with the following message:

No. of Pixels, (current $=n \times n)$ :

The value of $n$ defines the rectangular region that will be used to collect pixel (or image) information from the data buffers. 
Next, a cursor will be displayed on the RGB monitor over the active buffer. This cursor is repositioned by using the UP, DOWN, RIGHT, and LEFT arrow keys on the computer key pad. Concurrently, a window will open on the computer display that gives the following commands:

Set upper left of rectangle,

Set upper left of rectangle below,

Set upper left of rectangle to right,

Set lower triangle of main rectangle,

Set upper triangle of main rectangle.

The locations prompted by the system are shown schematically in Fig. 3. When the data has been properly entered, the following message is displayed:

Remember to update the current configuration file!

This reminds the user to update the current system configuration file (or to create one) so that the measurements can be recalled for later use. Note that the size of the rectangle displayed on the RGB monitor (schematically shown in Fig. 3) will reflect the value, $n$, entered by the user.

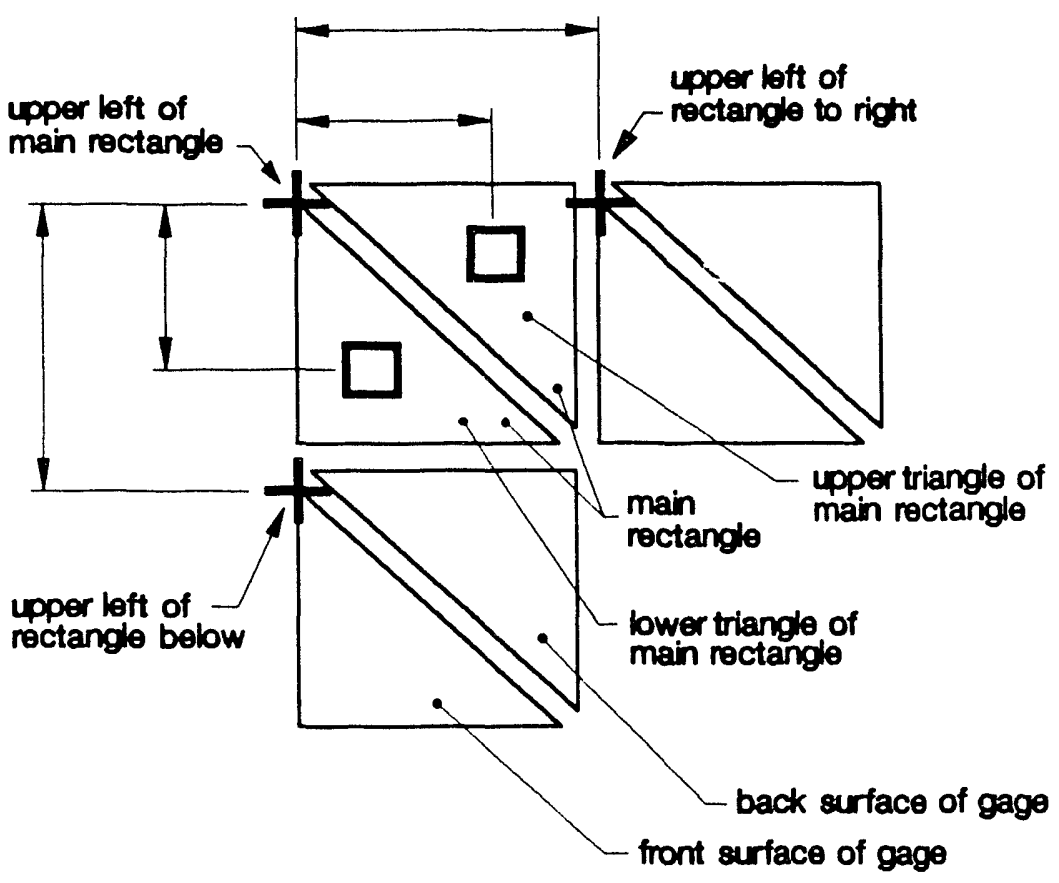

Fig. 3. Representation of the gage geometry and software cursor locations used with the Set Rectangle and Define Grid commands. 


\subsubsection{Define Grid}

The Define Grid option allows the user to set the $(x, y)$ coordinate array for the heatflux gage. An array definition must be in place to process the image data buffers. When activated, the following message will be displayed on the computer screen:

$$
\begin{aligned}
& \text { Press }<E N T E R>\text { to introduce new grid point, } \\
& \text { Press }<E S C>\text { to quit ... }
\end{aligned}
$$

The cursor will be displayed on the RGB monitor and controlled by the keypad cursor keys, as described previously. When the first point is set, the following message is displayed:

\section{Move Cursor to point $n$ of $N$}

where $n$ is the $n$th point entered out of a total of $N$ allowable points. For the current version, the total allowable points has been set to $N=100$.

The array must be rectangular (i.e., there must be $n \times m$ points defined for the sorting routines to work properly). Although the points can be entered in any order, they cannot be edited, and they cannot be triangular in distribution. If a bad data point is marked by the user, the option must be terminated and restarted to correct. When the points have been entered, the data should be saved in a grid file (*.GRD) through the FILES menu option.

\subsubsection{Calibrate}

The calibration data for the heat-flux gage system is measured and modified through the PROCES:/Calibrate submenu. Screen 10 shows the submenu display. The calibration procedure is mostly manual and depends on the user to generate the indicated parameters. The Point-Cal. option allows the imaging system to obtain the fluorescence data necessary to generate the required parameters.

\subsubsection{Parameter entry}

The parameters necessary to operate the heat-flux gage system were shown previously in Eqs.(2) and (5), which describe the temperature and heat-flux calculation. The Calibration submenu requires the following input:

$\begin{array}{lll}\text { Ratio(high) } & - & r_{h} \\ \text { (low) } & - & r_{l} \\ \text { Temp(high) } & - & T_{h} \\ \text { (low) } & - & T_{l} \\ \text { Cond (k) } & - & k \\ \text { Delta } & - & \Delta z_{P M P}\end{array}$

These values are obtained by measuring the ratio data at known temperature values (e.g., thermocouples mounted on a $\mathrm{Gd}_{2} \mathrm{O}_{2} \mathrm{~S}: \mathrm{Tb}$ coated gage). 


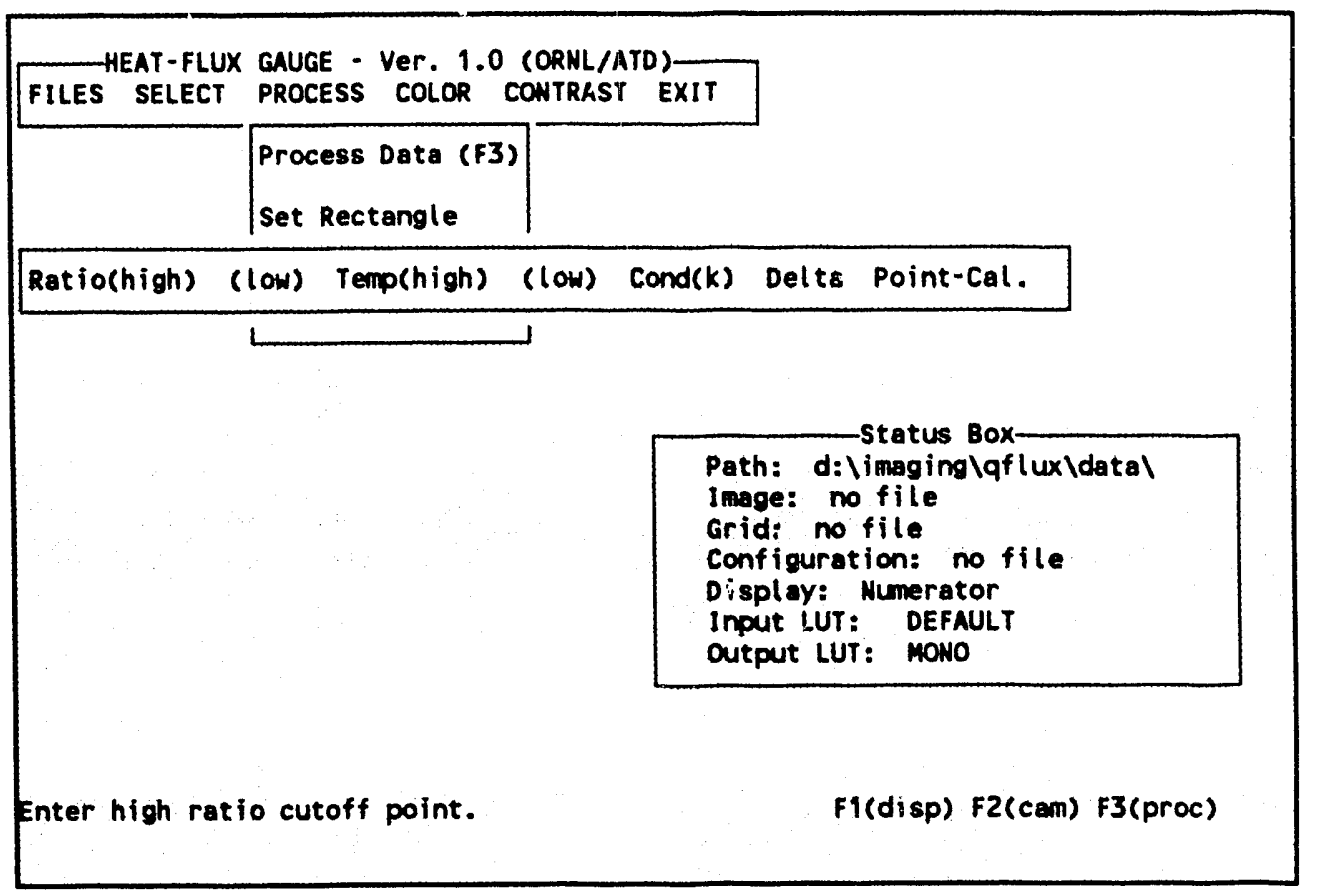

Screen 10. Calibrate submenu accessed through the PROCESS/Calibrate option.

\subsubsection{Point-Cal.}

The Point-Cal. option is activated to enable the system to measure the ratio values at known temperatures. When the Point-Cal. option is activated the following message is displayed:

\section{Set/Reset data ROI $(Y / N) ?$...}

The data $\mathrm{ROI}$ is a region of interest (ROI) that the user defines on the displayed data buffer from which to obtain fluorescence data. The Set/Reset message appear: each time the option is used so that the same region of an image can be accessed for data analysis as the image buffers are modified (i.e., for different temperature levels). When an ROI has been set and the ENTER key pressed, the system goes to the separate data buifers and calculates the background-corrected ratio in that region. The user must manually note the data and the thermocouple temperature for later determination of the curve-fit parameters, $r_{l}, r_{h}, T_{l}$, and $T_{h}$.

\subsection{COLOR MENU}

The COLOR menu option, shown on Screen 11, is not applicable to the temperature and heat-flux calculations for the current version of the program because of hardware limitations of the DT-2861 image processing device. 


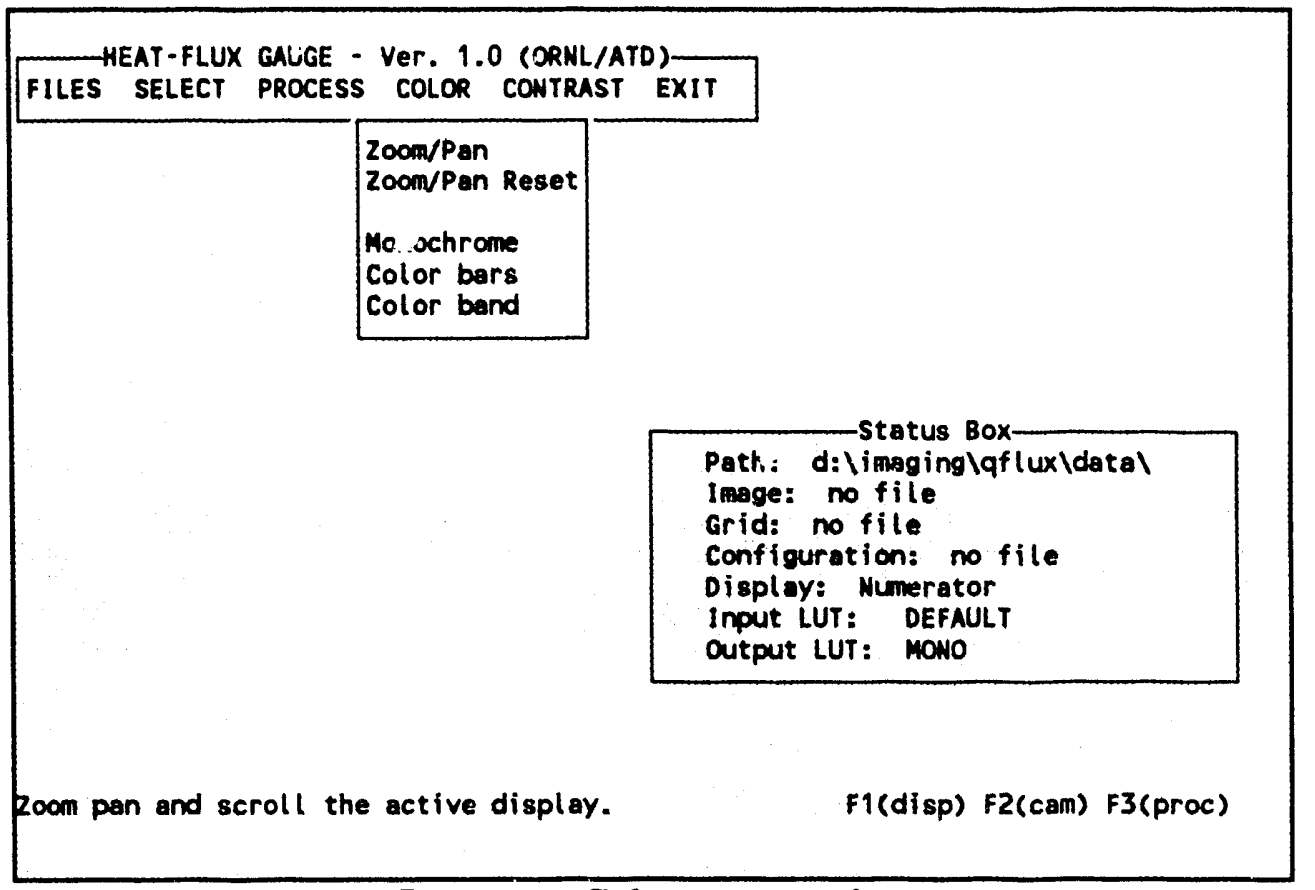

Screen 11. Color menu option.

\subsection{CONTRAST MENU}

The CONTRAST menu option, shown on Screen 12, is not applicable to the temperature and heat-flux calculations for the current version of the program because of hardware limitations of the DT-2861 image processing device.

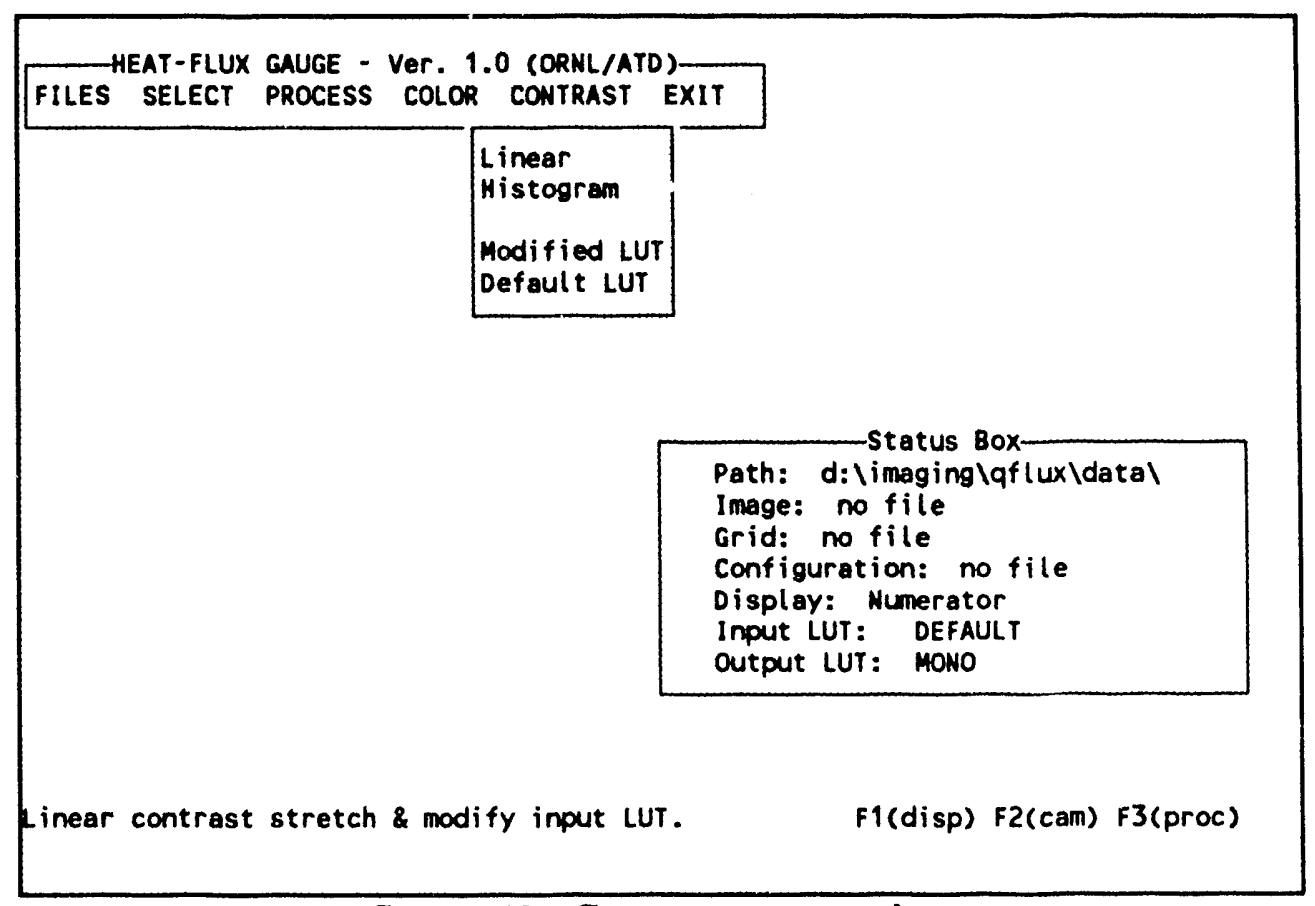

Screen 12. Contrast menu option. 


\subsection{PROGRAM EXIT}

The EXIT option on the main menu will allow the user to properly exit the software package. Screen 13 shows the exit window from which the user must verify his or her intent. The exit option can also be reached by pressing the ESC key anywhere in the main menu. If the user is several menus deep, repeated use of the ESC key will display the exit option.

When the program is exited, all data buffers and all grid and calibration definitions will be deleted from the DT-2861 memory. Therefore, the user must save all required data by stepping through the FILES menu option prior to exiting the program back to DOS (Screen 14).

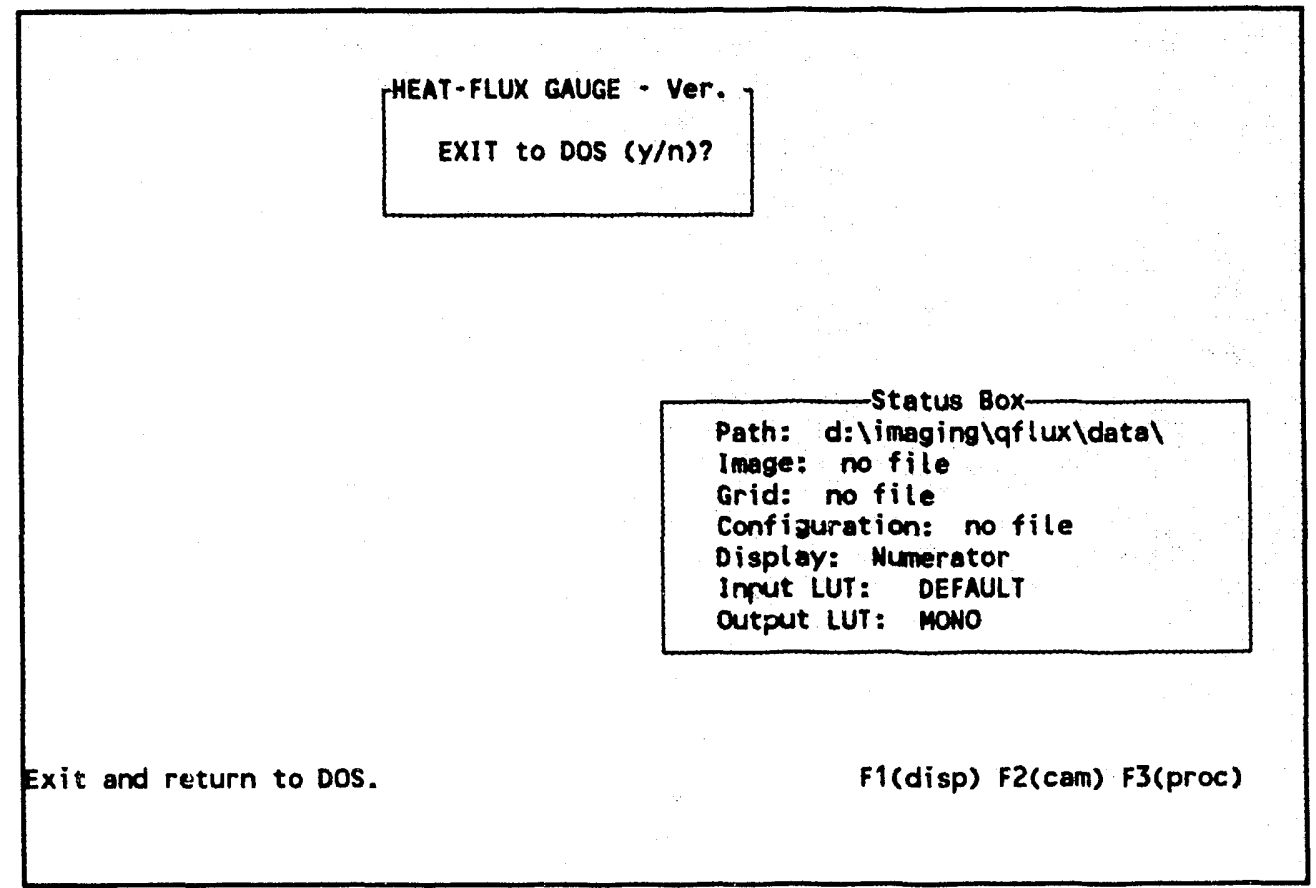

Screen 13. User is prompted to verify exit.

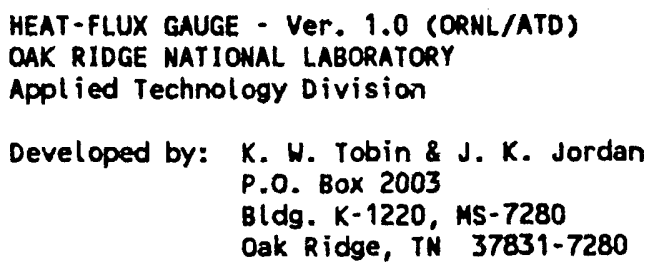

D:IIMAGING\QFLUX>

Screen 14. Program exited and back to DOS. 


\section{TUTORIAL}

This section will briefly outline the steps required to capture and process florescent emission data from the $\mathrm{Gd}_{2} \mathrm{O}_{2} \mathrm{~S}: \mathrm{Tb}$ phosphor-based heat-flux gage using the HEAT.EXE software package described herein.

STEP I Load the program by entering the command c: $1 . . \mid>$ HEAT

STEP II Choose the FILES/Set Path option and set the desired path for data storage and retrieval.

STEP III Select the SELECT menu.

Select the Display Numerator option.

Install the 415-nm narrow-band filter.

Turn on the ultraviolet light source.

Press the Camera (or F2) option.

While image is live, align the system, adjust the aperture, etc.

Press <ENTER > key when done with alignment

(Note: At this point no further realignment of the experiment hardware should be macie.)

Select the Display Numerator Background option.

Turn off the ultraviolet light source.

Press the Camera (or F2) option.

Press the <ENTER $>$ key when done.

Select the Display Denominator option.

Install the 490-nm narrow-band filter.

Turn on the ultraviolet light source.

Press the Camera (or F2) option.

Press the $<$ ENTER $>$ key when done.

Select the Display Denominator Background option.

Turn off the ultraviolet light source.

Press the Camera (or F2) option.

Press the <ENTER $>$ key when done.

Select the Display Numerator option.

(Note: This need only be done while the system is being set up on the first pass for the Set Rectangle and Define Grid commands in STEP IV.)

STEP IV Select the PROCESS menu.

Select the Set Rectangle option and follow the instructions.

Select the Define Grid option and set the grid coordinates. 
Select the Calibrate option if modification of the parameters is necessary.

STEP V Select the FILES menu.

(Note: Recall that when the program is exited all data will be lost; therefore, updates to the image data buffers and the grid and configuration should be made often.)

Select the Save Image option and enter a file name.

Select the Save Grid option and enter a file name.

Select the Save Config option and enter a file name.

STEP VI Select the PROCESS/Process Data (or F3) option. Enter a file name for the summary (*.SUM) and data (*.DAT) file when finished.

STEP VII To generate new temperature data, do the following:

Repeat STEP III above when the experiment is at a new steady-state temperature.

(Note: The image backgrounds (i.e., when the ultraviolet light source is off) will not vary with temperature; therefore, the image backgrounds do not need to be collected at every temperature point.)

Go to the FILES menu and save the new image data, if desired.

(Note: When the program is terminated, all data are lost unless previously saved to the current path from the FILES menu.)

STEP VIII EXIT the program when finished. Be sure to turn off the camera and ultraviolet light source. When the program is restarted at a later date for data collection, the STEP II procedures can be followed after the grid and configuration files have been loaded. Note that if the experiment geometry has changed even slightly, the entire procedure must be repeated. 


\section{REFERENCES}

1. Turley, W. D., et al., The Design and Characterization of a Prototype Opticai Heat-Flux Gauge, LA-11408-MS, UC-000, Los Alamos National Laboratory, Los Alamos, New Mexico, January 1989.

2. Tobin, K. W., et al., Evaluation of Thermographic Phosphor Technology for Aerodynamic Moc'el Testing, ORNL/ATD-40, Martin Marietta Energy Systems, Inc., Oak Ridge National Latoratory, Oak Ridge, Tennessee, August 1990.

3. Incropera, F. P., and D. P.Dewitt, Fundamentals of Heat Transfer, John Wiley \& Sons, New York, 1981, p.36. 
APPENDIX - SOFTWARE LISTING

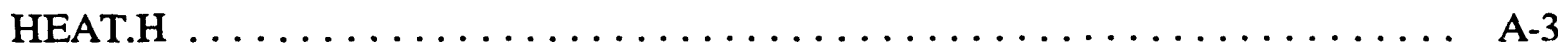

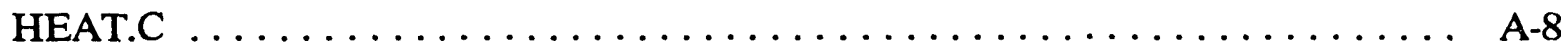

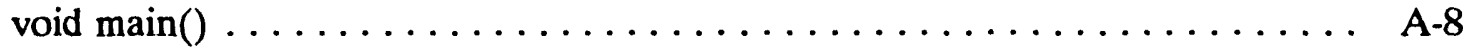

HEATMENU.C $\ldots \ldots \ldots \ldots \ldots \ldots \ldots \ldots \ldots \ldots \ldots \ldots \ldots \ldots \ldots \ldots \ldots \ldots \ldots \ldots$, A-9

void heatmenu ()$\ldots \ldots \ldots \ldots \ldots \ldots \ldots \ldots \ldots \ldots \ldots \ldots \ldots$ A-9

COUNT mQuit( VCMENU *mnuptr) $\ldots \ldots \ldots \ldots \ldots \ldots \ldots \ldots \ldots$ A-13

int Quit ()$\ldots \ldots \ldots \ldots \ldots \ldots \ldots \ldots \ldots \ldots \ldots \ldots \ldots \ldots \ldots \ldots \ldots \ldots, A, 13$

int GetYN( void $) \ldots \ldots \ldots \ldots \ldots \ldots \ldots \ldots \ldots \ldots \ldots \ldots \ldots \ldots \ldots \ldots \ldots, A, 13$

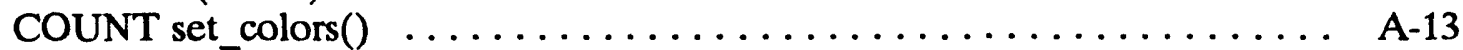

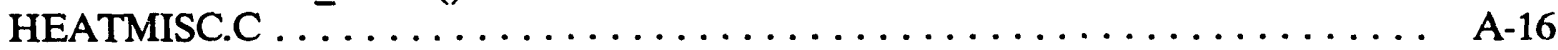

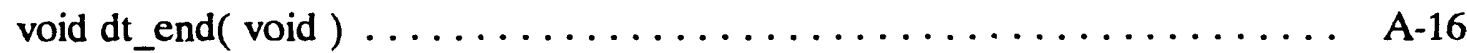

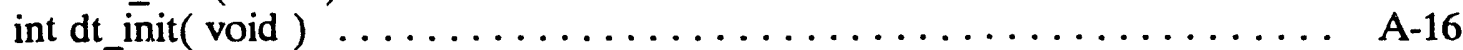

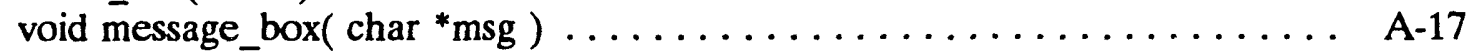

void message_box_np( char ${ }^{*}$ msg $) \ldots \ldots \ldots \ldots \ldots \ldots \ldots \ldots \ldots \ldots \ldots \ldots \ldots \ldots$, A-18

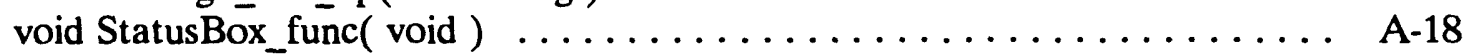

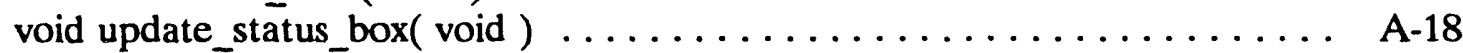

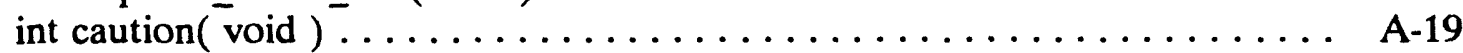

int dt_cursor(int *ptr_grey, int *ptr_row, int *ptr_col,

int row sensitivity, int col_sensitivity) $\ldots \ldots \ldots \ldots \ldots \ldots \ldots \ldots \ldots$ A-20

int F1_händler( COUNT key_code, VCPROKEY *ksp ) .......... A-21

int $\mathrm{F}_{2}$ handler( COL'VT key_code, VCPROKEY ${ }^{*}$ ksp ) . .......... A-21

int $\mathrm{F}^{-}$handler( COUNT key_code, VCPROKEY ${ }^{*}$ ksp ) $\ldots \ldots \ldots \ldots \ldots$ A-21

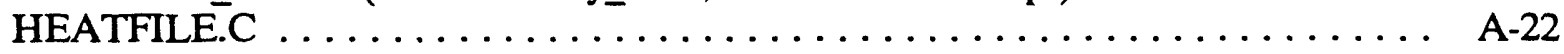

COUNT mPath( VCMENU *mnuptr $) \ldots \ldots \ldots \ldots \ldots \ldots \ldots \ldots \ldots$ A-22

COUNT mSave ( VCMENU *mnuptr ) $\ldots \ldots \ldots \ldots \ldots \ldots \ldots \ldots \ldots$ A-23

COUNT mRetrieve( VCMENU *mnuptr ) $\ldots \ldots \ldots \ldots \ldots \ldots \ldots \ldots$ A-23

int retrieve file( char *filename, int BufferNumber ) $\ldots \ldots \ldots \ldots \ldots \ldots$ A-24

int load_buffer( char *filespec, int BufferNumber ) $\ldots \ldots \ldots \ldots \ldots \ldots \ldots$ A-24

int save_buffer( char *filespec, int BufferNumber ) . . . . . . . .

int get_file_name_guts( char *title, char *prompt, char

*filename, char *defaultname) $\ldots \ldots \ldots \ldots \ldots \ldots \ldots \ldots \ldots \ldots \ldots \ldots \ldots \ldots \ldots$ A-24

char *add run ext( char *buf, int runnum ) $\ldots \ldots \ldots \ldots \ldots \ldots \ldots \ldots \ldots \ldots$ A-25

COUNT $\bar{m}$ SaveGrid( VCMENU *mnuptr ) $\ldots \ldots \ldots \ldots \ldots \ldots \ldots \ldots$ A-26

COUNT mRetrieveGrid( VCMENU *mnuptr ) $\ldots \ldots \ldots \ldots \ldots \ldots \ldots$ A-26

COUNT mSaveConfig( VCMENU *mnuptr $) \ldots \ldots \ldots \ldots \ldots \ldots \ldots \ldots$ A-27

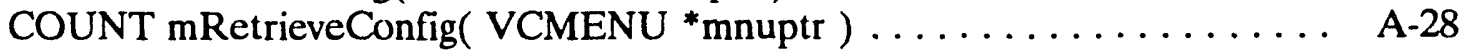

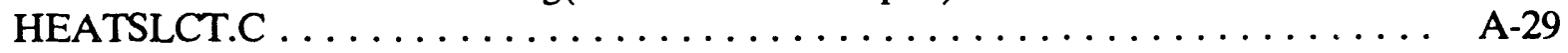

COUNT mNumerator( VCMENU * mnuptr ) $\ldots \ldots \ldots \ldots \ldots \ldots \ldots \ldots$ A-29

COUNT mNumeratorBack( VCMENU *mnuptr ) $\ldots \ldots \ldots \ldots \ldots \ldots \ldots$ A-29

COUNT mDenominator( VCMENU *mnuptr ) $\ldots \ldots \ldots \ldots \ldots \ldots \ldots$ A-29

COUNT mDenominatorBack( VCMENU *mnuptr ) $\ldots \ldots \ldots \ldots \ldots \ldots$ A-29

COUNT mRatio( VCMENU * mnuptr ) $\ldots \ldots \ldots \ldots \ldots \ldots \ldots \ldots$ A-29

COUNT mTemperature( VCMENU *mnuptr ) $\ldots \ldots \ldots \ldots \ldots \ldots \ldots$ A-30

COUNT mHeatFlux ( VCMENU *mnuptr $) \ldots \ldots \ldots \ldots \ldots \ldots \ldots$ A-30

COUNT mCamera( VCMENU *mnuptr ) $\ldots \ldots \ldots \ldots \ldots \ldots \ldots \ldots$ A 30

void switch_display ( int image number $) \ldots \ldots \ldots \ldots \ldots \ldots \ldots \ldots$ A-30 
int turn_camera_on( void $) \ldots \ldots \ldots \ldots \ldots \ldots \ldots \ldots \ldots \ldots \ldots \ldots \ldots \ldots$ A-30

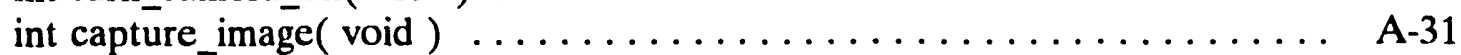

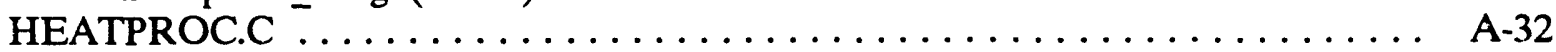

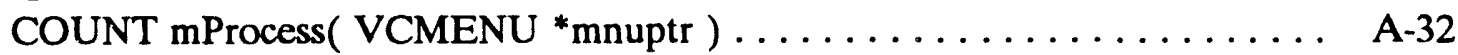

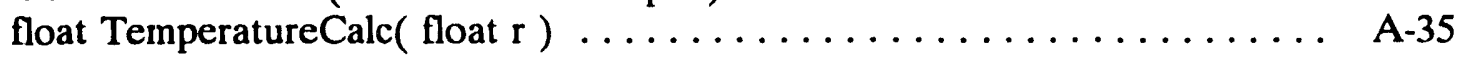

float HeatFluxCalc( float $T_{-}$top, float $T_{-}$bot $) \ldots \ldots \ldots \ldots \ldots \ldots \ldots$ A-35

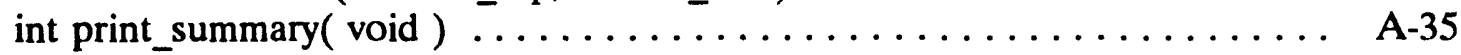

COUNT mSetRectangle( VCMENU *mnuptr ) ............. A-36

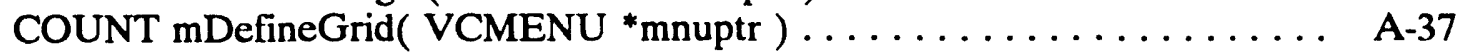

int arrange( int $x y[][2]$, int npts, int maxrows, int *mrows

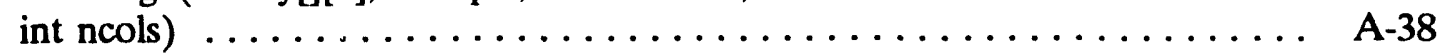

int mark_grid( int image_number, int mark_row, int mark_col,

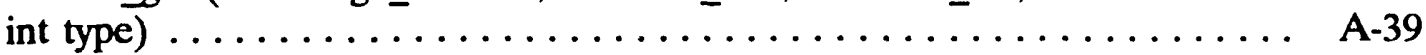

int draw_square( int image_number, int square_row, int

sqaure col $\ldots \ldots \ldots \ldots \ldots \ldots \ldots \ldots \ldots \ldots \ldots \ldots \ldots \ldots$ A-40

int mark_display( int image_number, int mark_row, int

mark_col, char ${ }^{*}$ title_msg, char ${ }^{*}$ mark_msg) $\ldots \ldots \ldots \ldots \ldots \ldots$ A-40

COUNT mEnterRatio ${ }^{-}$High ( VCMENU ${ }^{*}$ mnuptr $) \ldots \ldots \ldots \ldots \ldots \ldots \ldots$ A-40

COUNT mEnterRatioLow( VCMENU ${ }^{*}$ mnuptr ) $\ldots \ldots \ldots \ldots \ldots \ldots \ldots$ A 4 .41

COUNT mEnterTempHigh( VCMENU *mnuptr ) . . . . . . . . . A-41

COUNT mEnterTempLow ( VCMENU *mnuptr ) . . . . . . . . . A A-42

COUNT mEnterk( VCMENU *mnuptr ) . . . . . . . . . . . . A 4 42

COUNT mEnterx( VCMENU *mnuptr ) ............... A-42

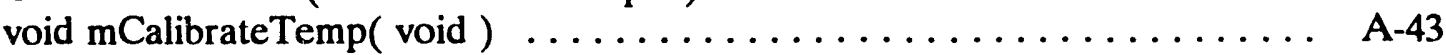

HEATCOLR.C . . . . . . . . . . . . . . . . . . . . . . . . . . A 45

COUNT mZoomPanReset( VCMENU *mnuptr ) ........... A-45

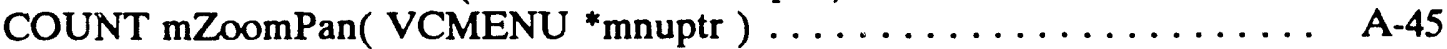

COUNT mMonochrome ( VCMENU *mnuptr ) .............. A-46

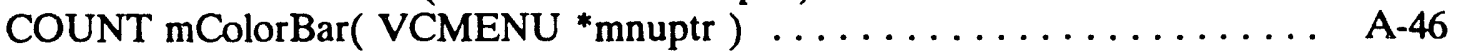

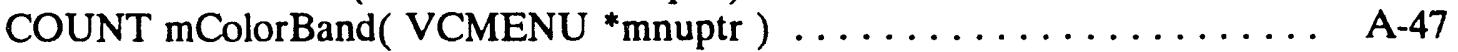

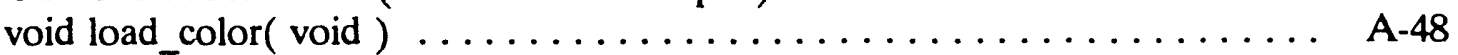

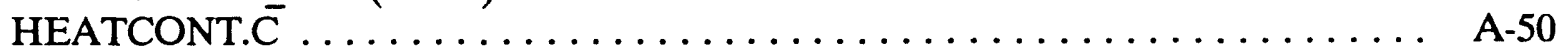

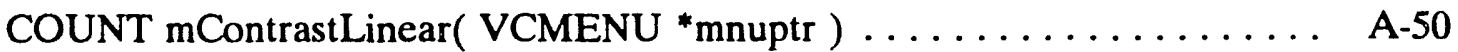

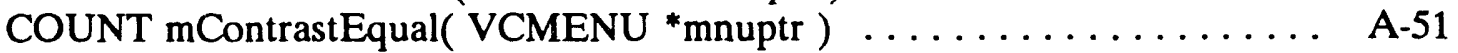

COUNT mSelectModLUT( VCMENU *mnuptr ) $\ldots \ldots \ldots \ldots \ldots \ldots \ldots$ A-51

COUNT mSelectDefLUT( VCMENU *mnuptr $) \ldots \ldots \ldots \ldots \ldots \ldots \ldots$ A-51 


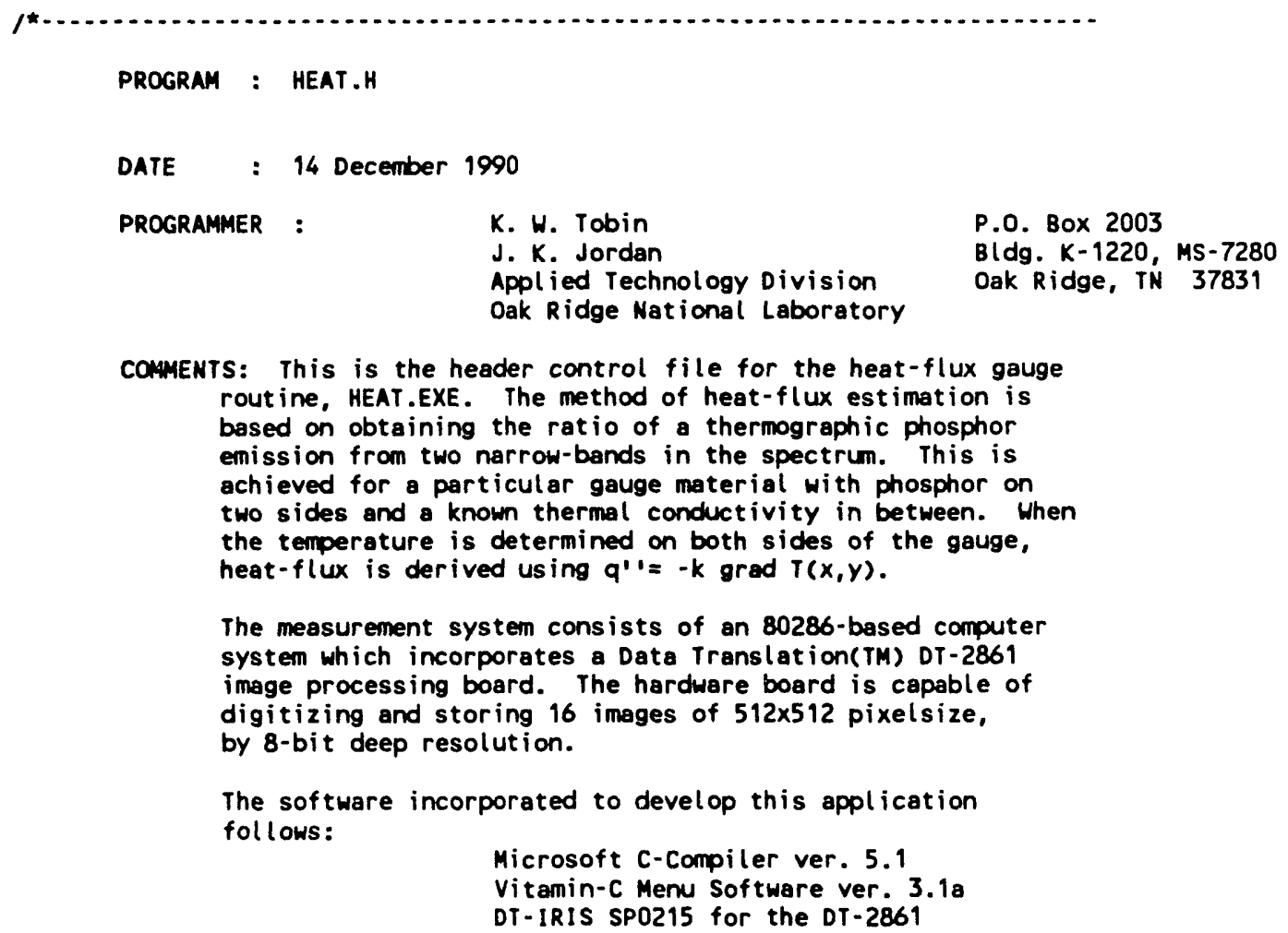

/* Include files for Data Translation, Vitamin-C, and Microsoft C5.1. "/

\#include <iserrs.h>

$I^{*}<-$ Dr2861 control files. */

$\#$ include <isdefs.h>

$I^{\star}<-$ Vitamin-c control file. */

\#include <vestdio.h>

/* <-. Microsoft C5.1 control files. */

\#include <string.h>

\#include 〈dos.h>

$\#$ include <math.h>

/* Global definitions for Data Translation associated hardware (DT2861). */

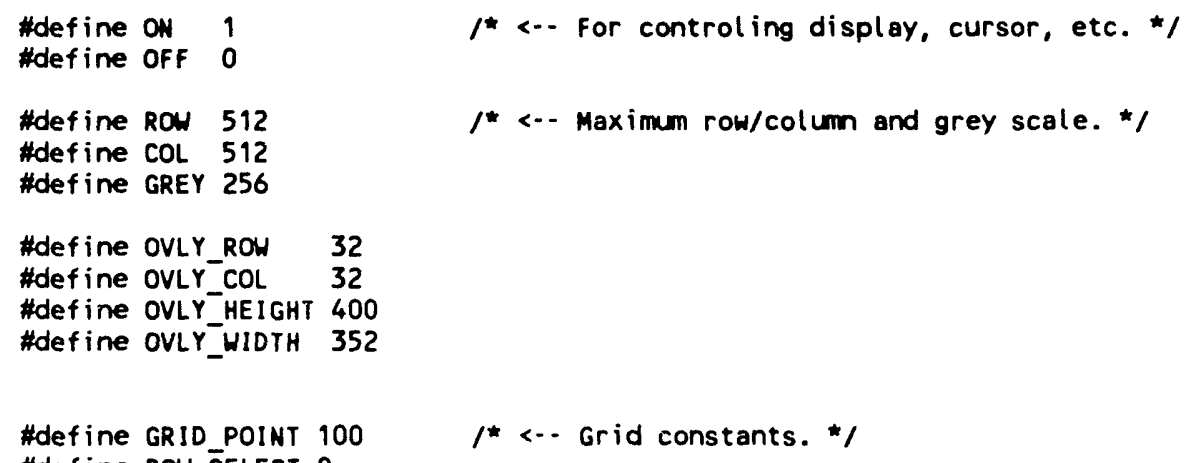


*define POINTS 5 \#def ine TOPROH 0.55

\#define TOPCOL 0.24

*define BOTROW 0.25

*define BOTCOL

1* Number of pixels to avg. about cursor (PxP array). */ 1 * Offsets for gathering data relative to grid. */ /* TOP... refers to top phosphor.

1 * BOT... refers to botton phosphor.

* (these are system defaults)

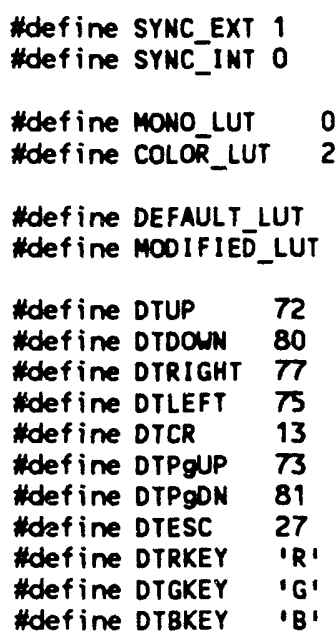

$$
\text { I* <- internal/external sync control */ }
$$$$
I^{*}<\text { output look-up-table definitions */ }
$$$$
f^{\star}<- \text { input look-up-table definitions */ }
$$$$
I^{\star}<-- \text { for controling the cursor } \star /
$$

\#define TOTAL_IMAGES 7 "define DATA_IMAGES 4

* <- number of defined selectable buffers */

"define NUMERATOR

\#define NUMERATOR_BACK

\#define DENOMINATOR

\#def ine DENOMINATOR_BACK

"define RATIO

"define TEMPERATURE

"define HEAT_FLUX

$$
1 \text { * <- number of data images */ }
$$

"define NUMERATOR CORR

\#define DENOMINATOR_CORR

\#define GRAPHIC_BUF \#define OVLY_BUF

$$
I^{\text {* }} \text { - - image buffers */ }
$$

/ Global definitions for DOS system */

\#define SUCCESS 0

$$
l^{*} \text { <- Subroutine test parameter. */ }
$$

*define FAILURE 1

\#define PATHSTRLEN 28

"define FILENAMLEN 9

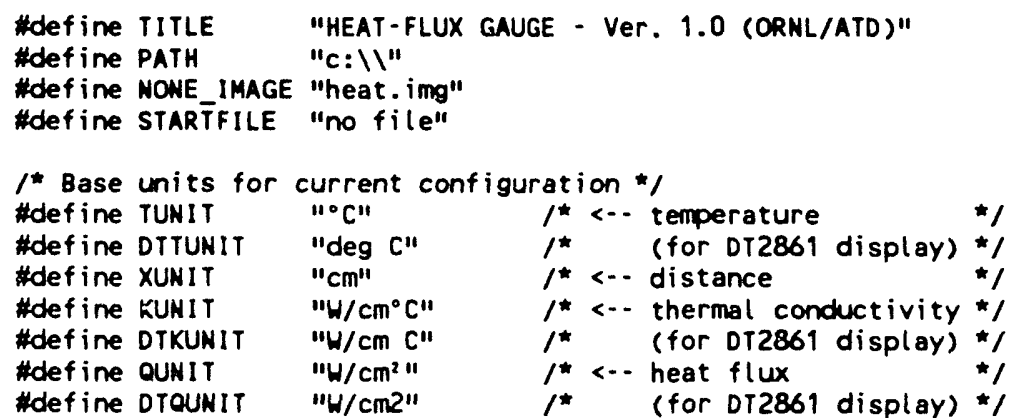




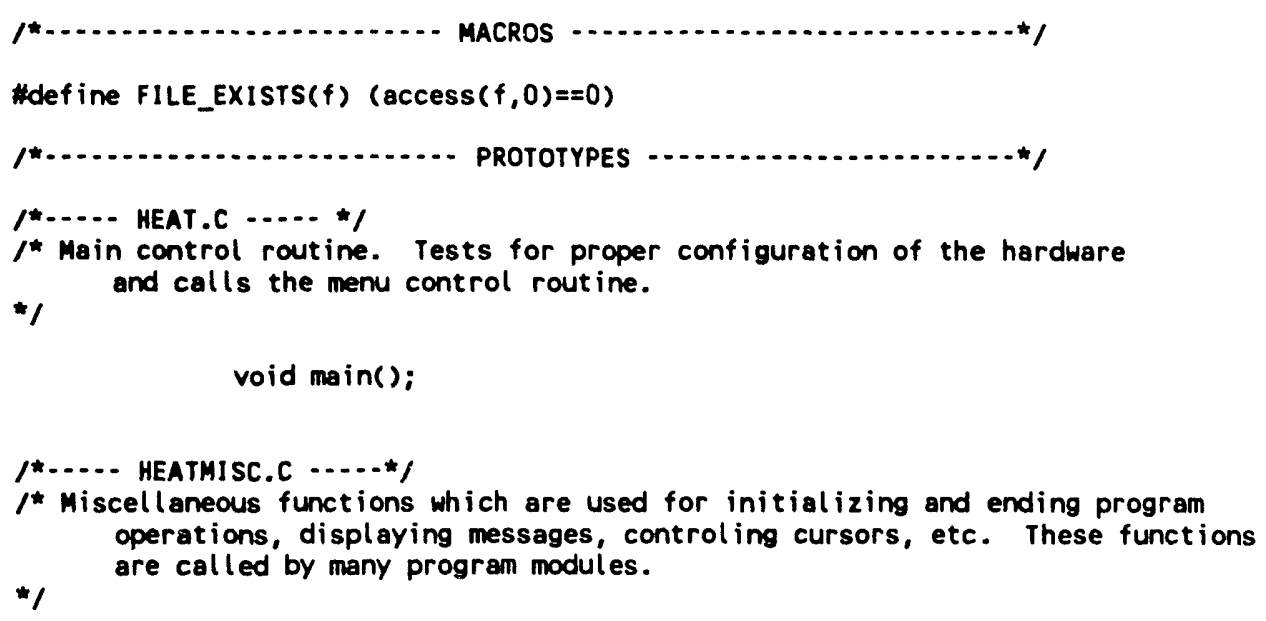

void heatmenu( void );

COUNT mauit( VCMENU *moptr );

int Quit ( void);

int GetYN( void);

COUNT set_colors( void);

HEATFILE.C .....*/

$l^{\star}$ Functions for disk file operations such as retrieving and saving data

* to and from the indicated drive path.

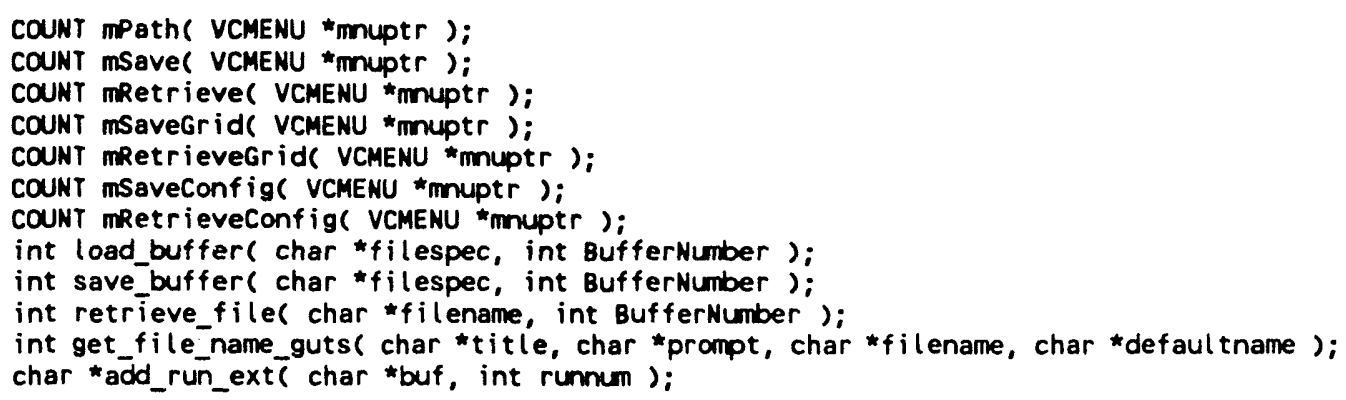




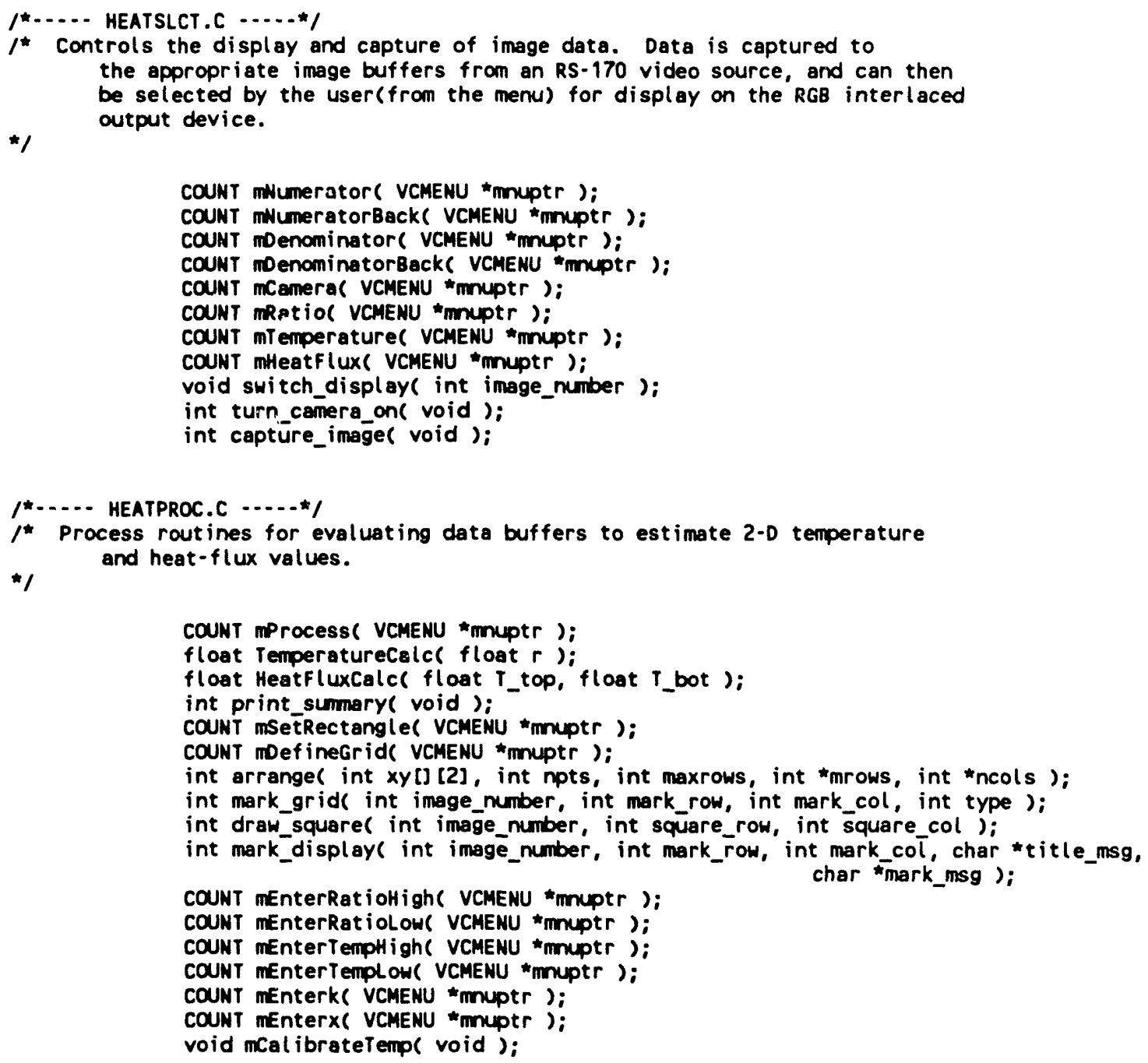

COUNT MProcess( VCMENU *mnuptr)

float Temperaturecelc( float $r$ ):

float HeatfluxCalce float T_top, float T_bot );

1* Utility functions for producing false-color output of the displayed data using the DT-2861 ouput look-up-tables (LUT'S). Also allows for zoom, pan, and scroll of the displayed data.

$\star 1$

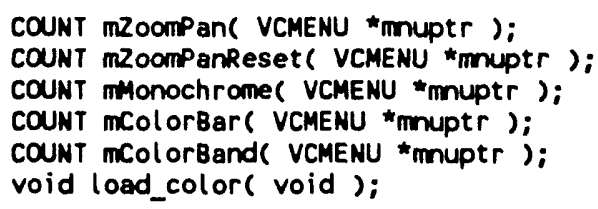

$1^{*}$ Functions contained here allow for modification and control of the input LUT's. Both a linear mapping and a statistical mapping (based on histogram equalization) are included. Once these mappings are defined they can be enabled or disabled at any time.

$\star 1$

COUNT mContrastLinear( VCMENU *mnuptr)

COUNT mContrastEqual ( VCMENU *mnuptr );

COUNT mSelectModLUT( VCMENU *mnuptr ):

COUNT mSelectDefLUT( VCMENU *muptr ); 
WINDOW VARIABLES $\ldots \ldots \ldots \ldots \ldots \ldots$

COUNT StatusBoxHindow;

$l^{*}$ program status information */

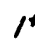
GLOBAL VARIABLES $\ldots \ldots \ldots \ldots \ldots \ldots \ldots$ /

char Path [PATHSTRLEN+1];

" - - display environment */

char Fi leName [F ILENAMLEN], Cal Name [F ILENAMLEN]

Grid'Nane [FILENAMLEN];

char BufferName [TOTAL_IMAGES] [15] ;

char ILUTName [7] [10], OLUTMane [7] [10];

int ActiveBuffer:

/* <- Dr2861 environment */

int ActivelLUT, ActiveOLUT;

int red_lut [GREY], green_lut [GREY], blue_lut [GREY];

int GridPoints,GridRow,GridCol;

int grid[GRID_POINT] [2];

int top grey[GRID_POINT] [2],

bot grey [GRID POINT] [2];

float top_ratio[GRID_POINT].

bot_rattio[GRID_POINT];

float top_temperature [GRID_PŌINT]

float heat_flux [GR'ID_POINT];

bot_temperature[GRID_POINT];

int grid_points;

1 *- grid variables for data collection */

float grid toprow, grid_topcol;

float grid_botrow, grid_botcol;

float T_high, T_low; $\quad /^{*}$ temperature extremes, <-. calibration data */

float $r_{-}$high, r-low; $\quad 1^{*}$ ratio extremes,

1 * <- process enviromment */

* ( [] [2] $=\mathrm{num} /$ den select $) * /$

$f$ loat k_cond, delta_x:

$f^{*}$ thermalconductivity \& gauge thickness. 


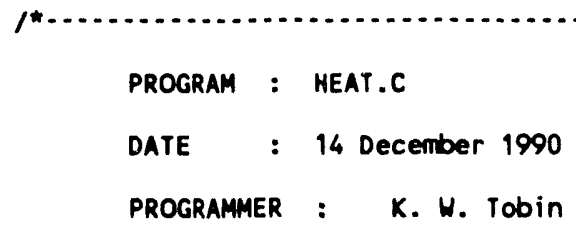

ROUTINES
J. K. Jordan

Applied Technology Division

Oak Ridge National Laboratory

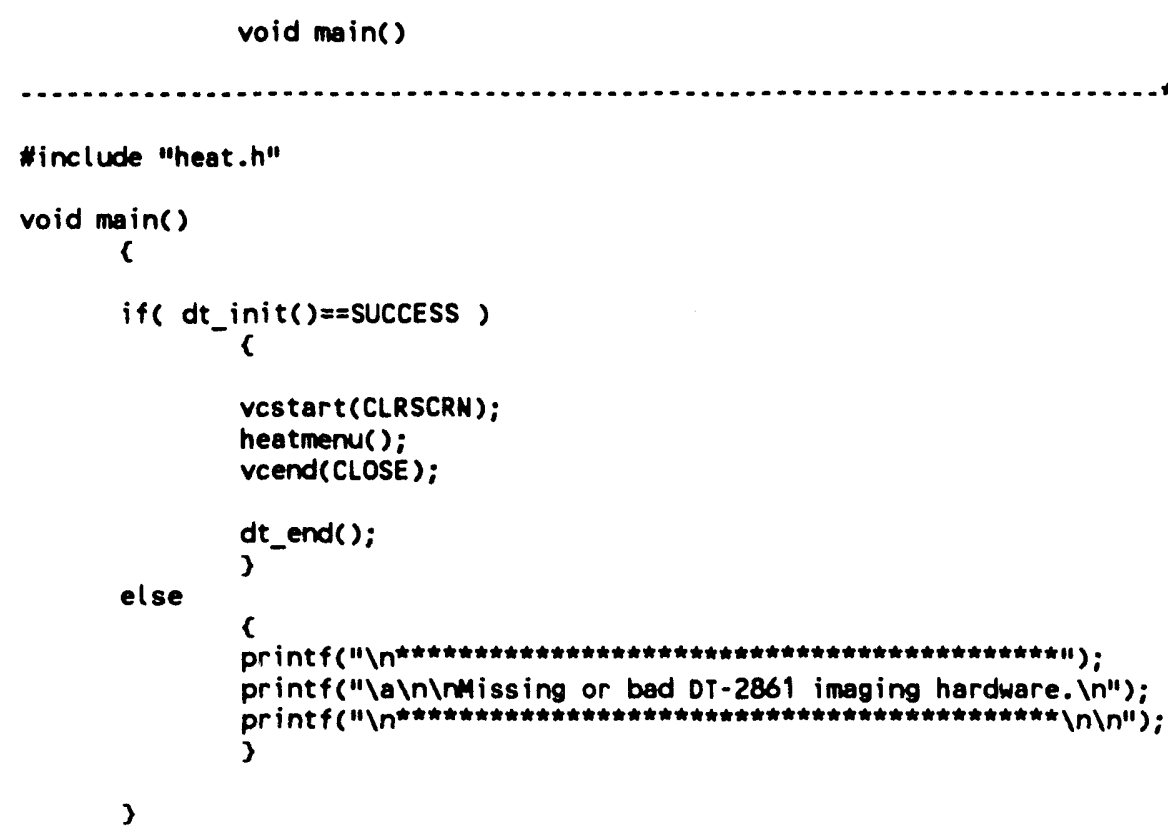




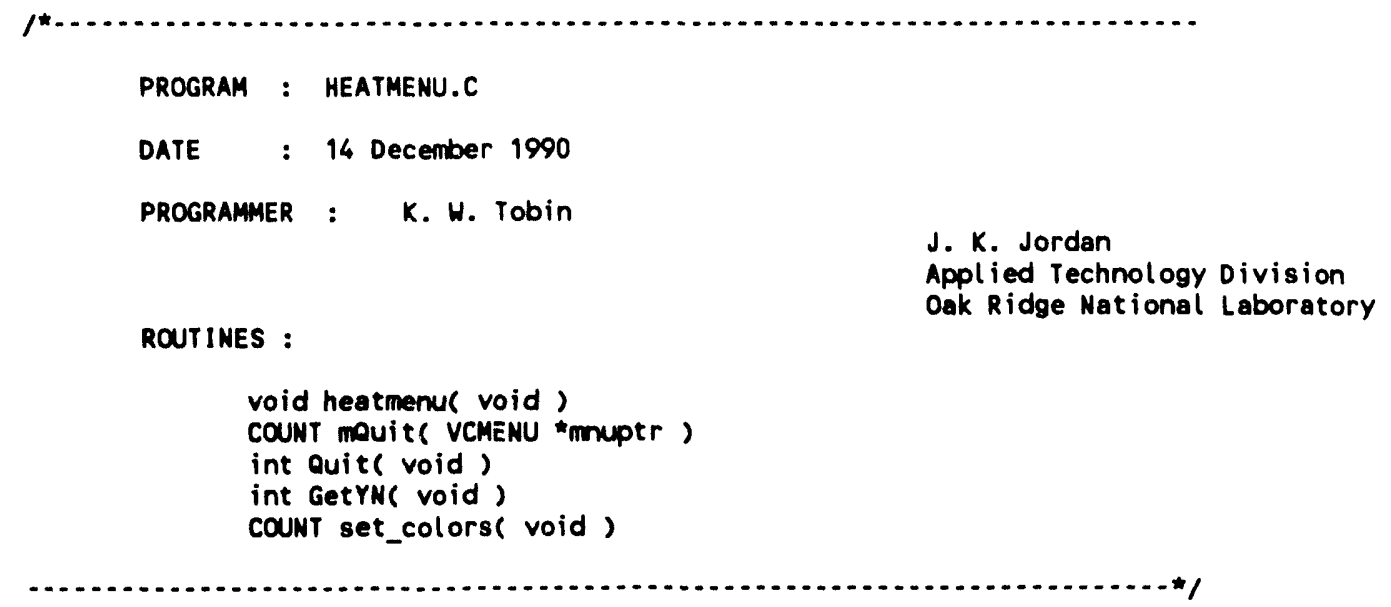

"include "heat.h"

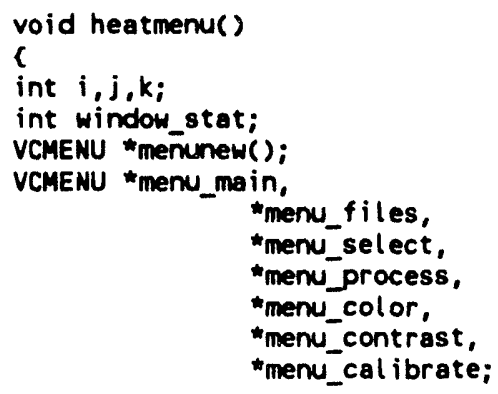

$I^{\star}$ SET UP SCREEN MENU CONTROL USING VITAMIN-C ROUTINES . . . *

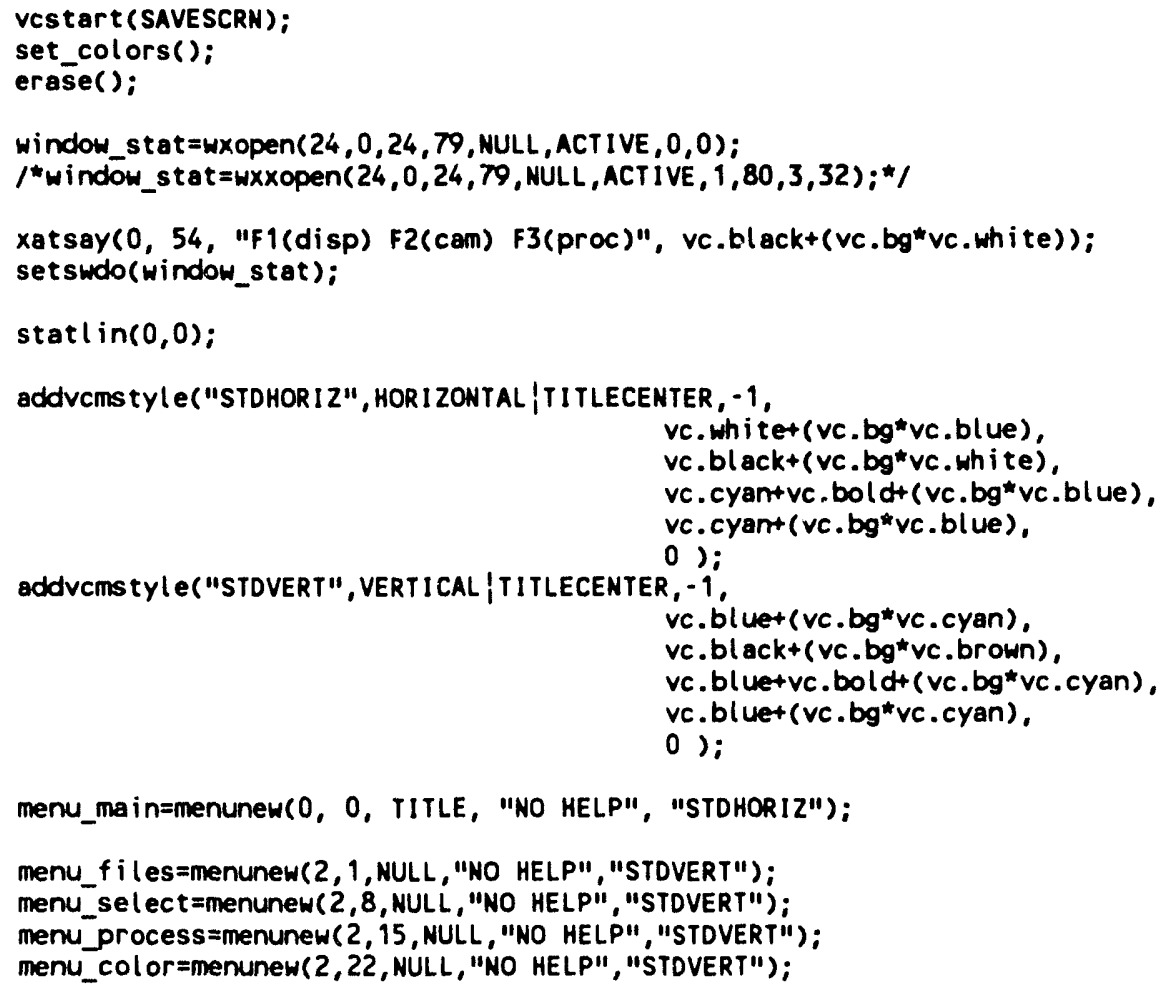




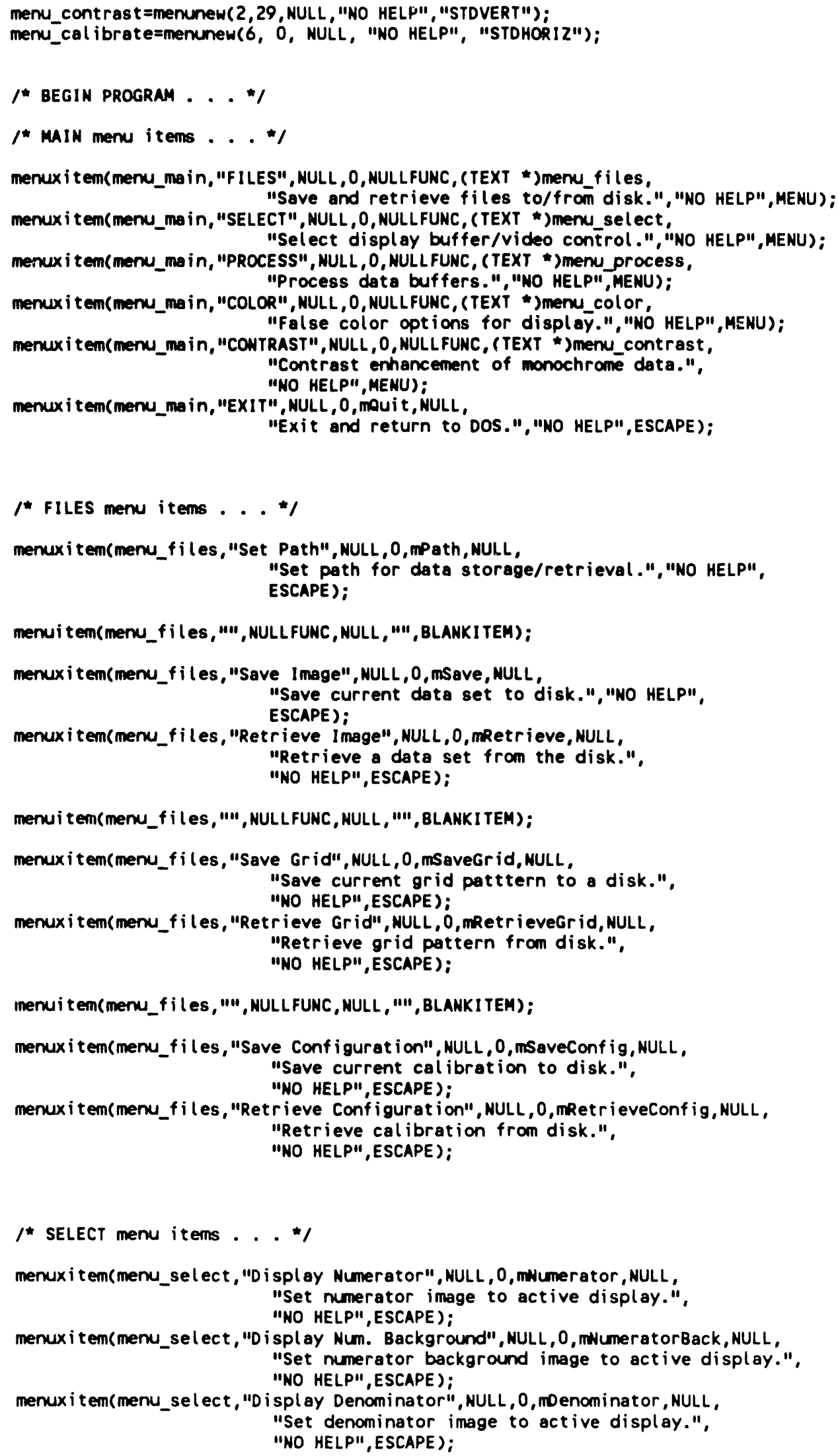




\section{A-11}

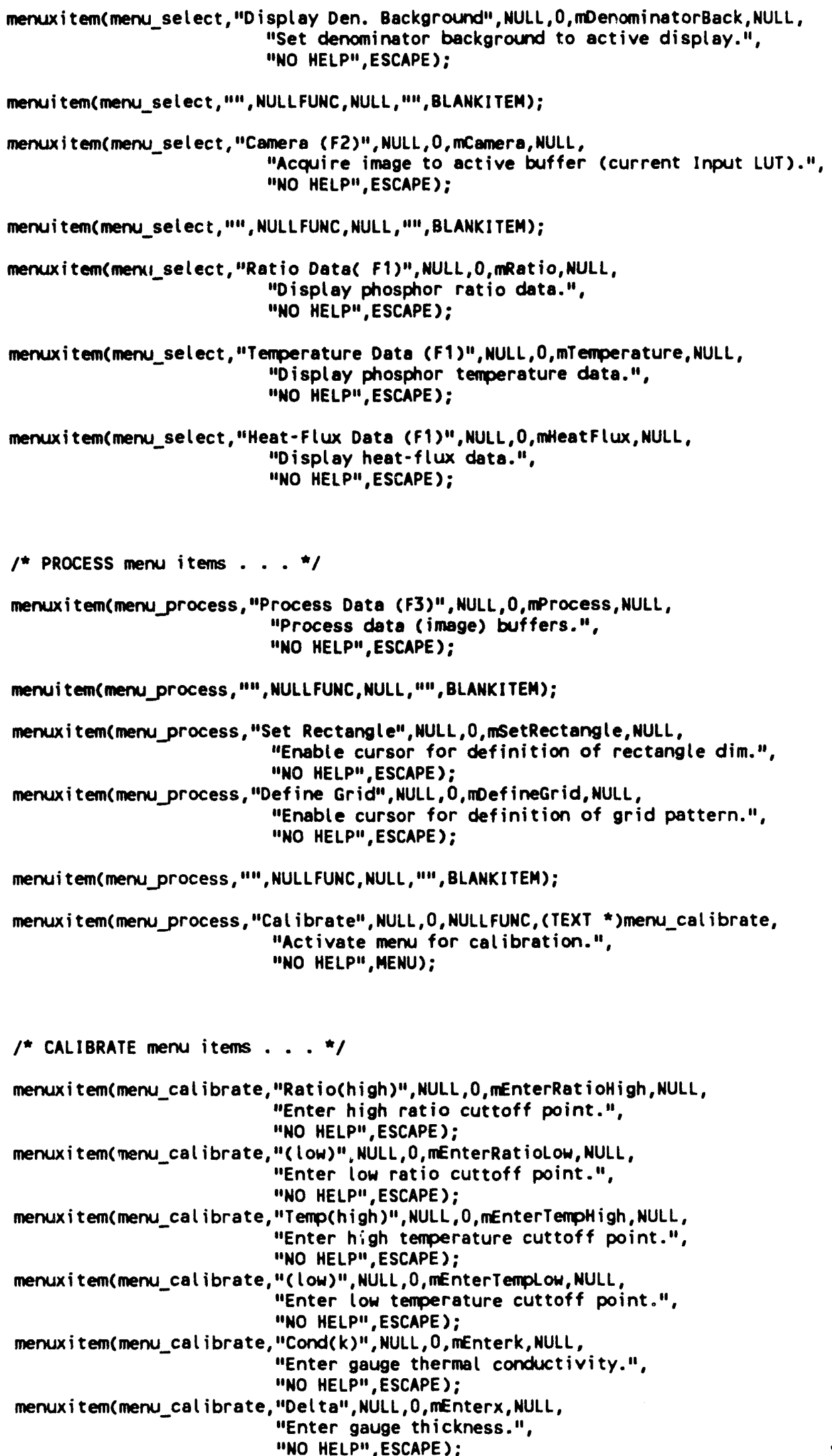




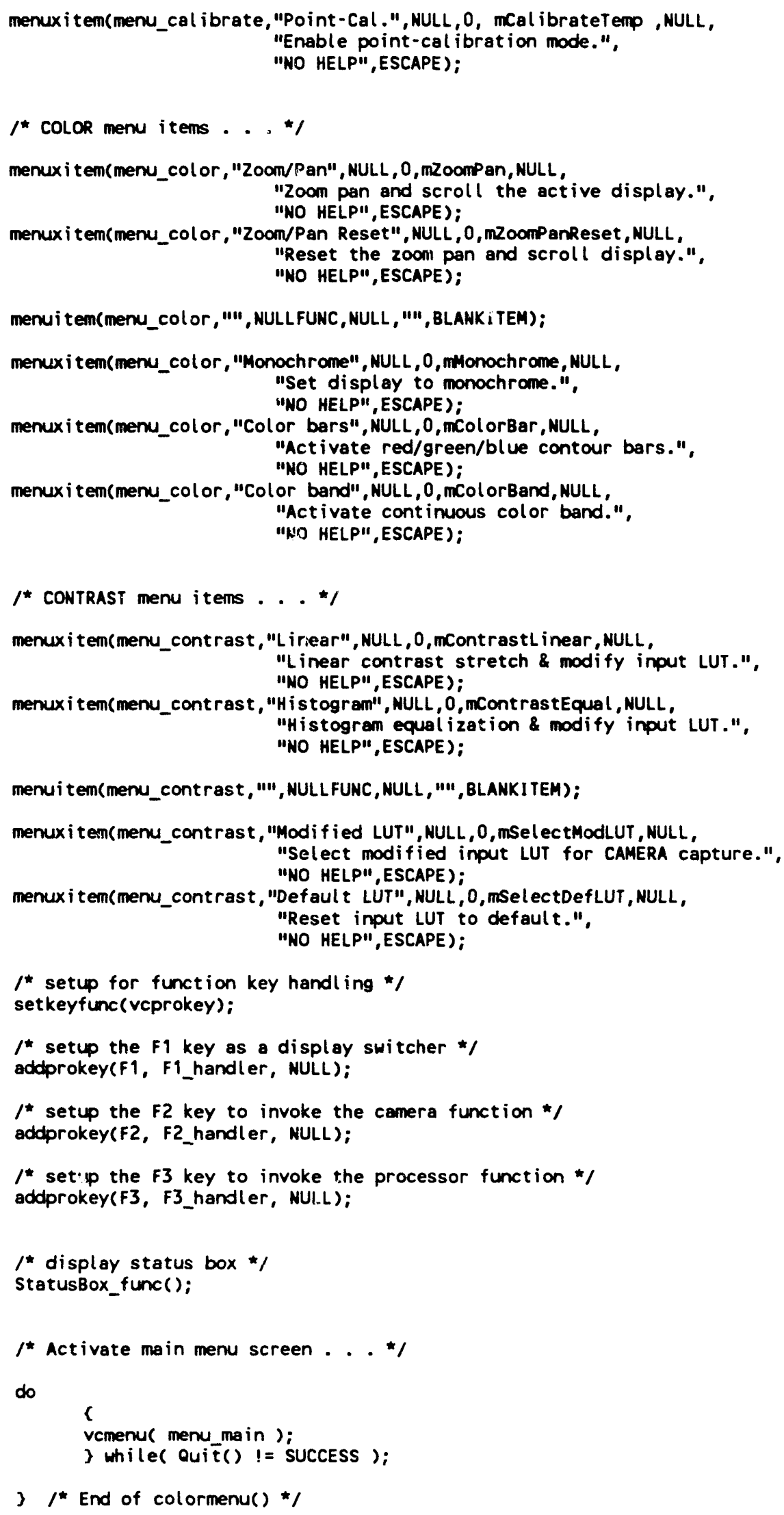




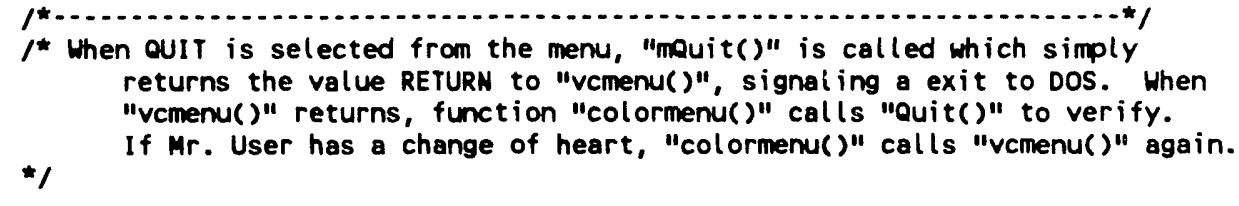

COUNT mQuit( VCMENU *muptr)

c

3

return RETURN;

/*

$1^{*}$ does he really want to quit or was this an accident? */

$f^{*}$ Quit () is called from main to verify he actually wants to quit */

int Quit()

c

int wtemp;
char c;
int Retcode;

wtemp=wxxopen $(2,23,6,47, " '$, , ACT IVE |BORDER | BD2 , 0, 0,5,32);

if ( wtemp $==-1$ ) erase();

terror("Unable to open QuitWindow. Aborting");

xatsay( 1,3, "EXIT to DOS $(y / n)$ ?",ve.df $(t)$;

if $((c=\operatorname{GetYN}())==$ ESC $)$

$C=$ 'N';

if $c=={ }^{\prime} y$ ' If $c==$ ' $y$ ',

else

RetCode=SUCCESS;

RetCode=FAILURE;

wclose (wtemp);

了

return RetCode;

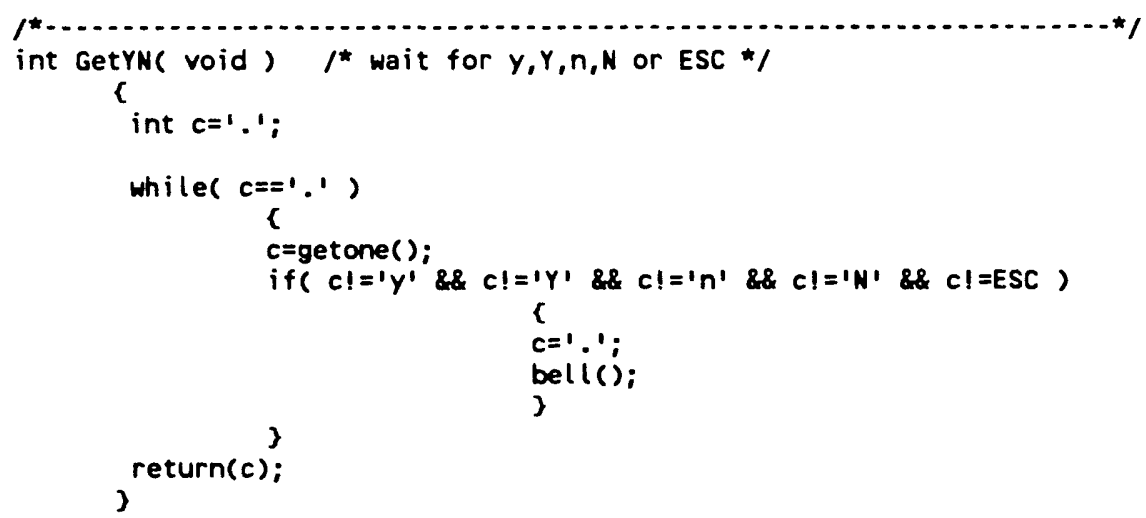


1 * Color Set $1 * 1$ wtable[1].bd_t = wtable [1].bg_t = wtable[1].say_t $=$ wtable [1].nget_t = wable [1].get $\bar{t}=$ wtable[1].tit t

1* Color set 2 * wtable [2].bd $t=$ wtable [2].bg_t = wtable [2]. say $t=$ wtable [2].nget_t = wtable [2].get_t $\bar{t}=$ wtable [2].tit t =

$1 *$ Color set $3 \star 1$ wtable [3] . bd $t=$ wtable [3] . bg_t wtable [3] . say_t wtable [3] .nget_t = wtable [3].get_t $=$ wtable [3].tit_t

1* Color Set $4 * 1$ wtable[4]. bd_t = wtable[4].bg_t wtable[4]. say_t = wtable [4].nget $t=$ wtable [4].get_t $=$ wtable[4].tit t

* Color set 5*/ wtable [5] . bo $t=$ wtable[5].bg_t = wtable [5].say_t = wtable [5].nget $t=$ wtable [5].get $\bar{t}=$ wtable[5].tit_t =

1* Color Set 6*/ wtable [6].bd t wtable[6].bg_t $=$ wtable[6].say_t $=$ wtable [6]. nget $t=$ wtable[6].get $\bar{t}=$ wtable[6].tit_t $=$

/ Color Set 7*/ wtable [7].bd t wtable [7]. bg t $=$ wtable[7].say_t = wtable[7].nget_t = wtable[7].get $\bar{t}=$ wtable[7].tit t $^{-}=$

/* Color set 8 */ wtable [8] .bd $t=$ wtable [8].bg_t = wtable[8] $\cdot$ say_t $=$ wtable [8]. nget $t=$ wtable [8].get $\bar{t}=$ wtable[8].tit_t = vc.black+(vc.bg*vc. white):

$v c$. browntvc.boldt (vc. bg*vc.blue);

vc. browntvc.bold+(vc.bg*vc.blue);

vc. white+vc.bold+(vc. bg*vc. blue):

vc.black+(vc.bg*vc. White);

vc.black+(vc.bg*vc. white);

vc. cyantvc.bol $d+$ (vc. bg*vc. blue);

vc. cyantvc.boldt (vc. bg*vc.blue)

vc.cyantvc.boldt (vc.bg*vc.blue);

vc. white+vc.bold+(vc.bg*vc.blue);

vc.black+(vc.bg*vc. White);

vc. white+vc.bold+(vc.bg*vc.blue);

vc. Whi te+vc.boldt(vc, botvc. red);

vc.redt (vc.bg*vc. white):

vc.black+(vc.bg*vc. white)

vc.black+(vc.bg*vc. white)

vc. whitervc.bold;

vc.browntvc.boldt (vc. bg*vc.red);

vc.browntvc.bold+(vc.bg*vc.blue);

vc.bluet (vc.bg*vc.cyan);

vc.bluet (vc.bg*vc.cyan)

ve.black+(vc.bg*vc.cyan);

vc. White+yc.bold;

vc. bluet (vc.bg*vc.cyan)

vc. Whitetvc.boldt (vc.bg*vc. red)

vc. White+vc.boldt (vc.bg*vc.red);

vc. whi te+vc.boldt (vc.bg*vc. red)

vc.browntvc.boldt (vc.bg*vc. red)

vc. brountvc.bold;

$v c$. whitetvc. boldt (vc. bg*vc. red);

vc.red+ (vc.bg*vc.brown)

vc. browntvc. boldt (vc. bg*ve. brown); vc. browntvc. boldt (vc. bg vc. brown); vc. whitetvc.bold+(vc.bg*vc. brown);

$v c$. greentvc.bold;

vc.browntvc.boldt(vc.bg*ve. brown);

vc.browntvc.boldt(vc.bg*vc.brown);

vc.black+(vc.bg*vc.green);

$v c . b l a c k+\left(v c . b{ }^{*} v c . g r e e n\right)$;

$v c$. whi te+vc. boldt (vc. bg*vc.green);

vc.browntvc.bold:

vc.black+(vc.bg*vc.brown);

vc browntvc bol dt (vc.bo*vc. white);

vc. white+vc. bold+ (vc. bg*vc. magenta);

vc. whi te+vc.bold+ (vc. bg*vc. magenta)

vc. browntvc.boldt (vc.bg*vc.magenta)

vc. browntvc.bold;

$v c$. magenta+ (vc.bg*vc. white) 


\section{A-15}

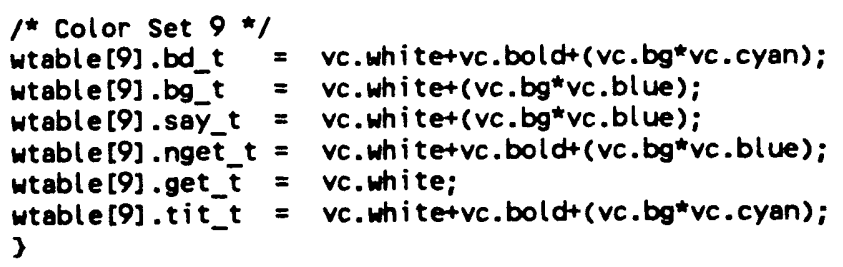




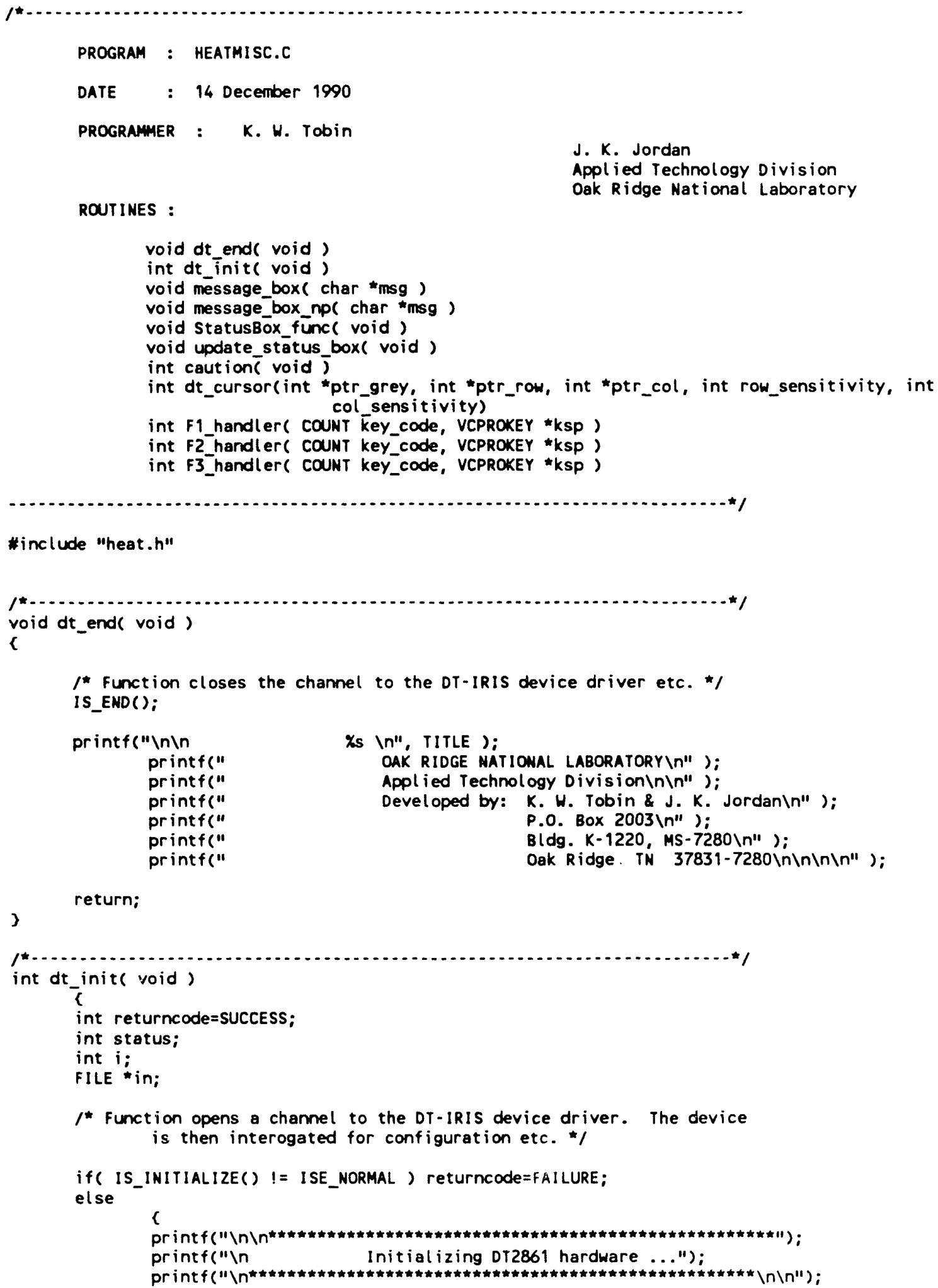

\#include "heat.h" 


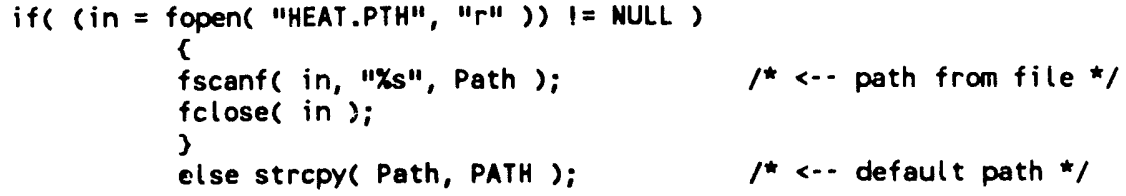

sprintf( BufferName [0], "Numerator" ); sprintf( BufferName [1], "Num. Back." ); sprintf( BufferName [2], "Denominator"); sprintf( BufferName [3], "Den. Back." ); 


\section{A-18}

int pwidth = strlen(prompt);

int mwidth $=\operatorname{strlen(msg)}$;

$1^{*}$

width = midth;

if ( width<pwidth)

width=pwidth:

width $+=7$

$\star /$

width $=\max ($ midth, pwidth $)+7$

start $=(80-$ width $) / 2$;

wtemp=wxxopen( 6 , start , 12, start+width, "11",ACT IVE |BORDER |BD2, 0,0,5,32);

if ( wtemp $=-1$ )

terror("Unable to open message_box. Aborting");

erase();

atsay (1, (width-mwidth)/2,msg);

atsay (3, (width-pwidth)/2,prompt);

getone():

wclose (wtemp)

了

$f^{\star}$ open a window and display a message */

$l^{*} \quad$ wait for a keypress (no prompt), then return */

void message_box_np( char *msg )

c

int width, start, wtemp;

width $=\operatorname{strlen(msg)}+7 ;$

start $=(80-$ width $) / 2$;

wtemp=wxxopen(6, start, 10, start+width, "' , ACTIVE |BORDER |BD2, 0, 0,5,32);

if ( wtemp $==-1$ )

terror("Unable to open message_box. Aborting");

erase():

atsay $(1,4, \mathrm{msg})$;

getone():

welose (wtemp);

)

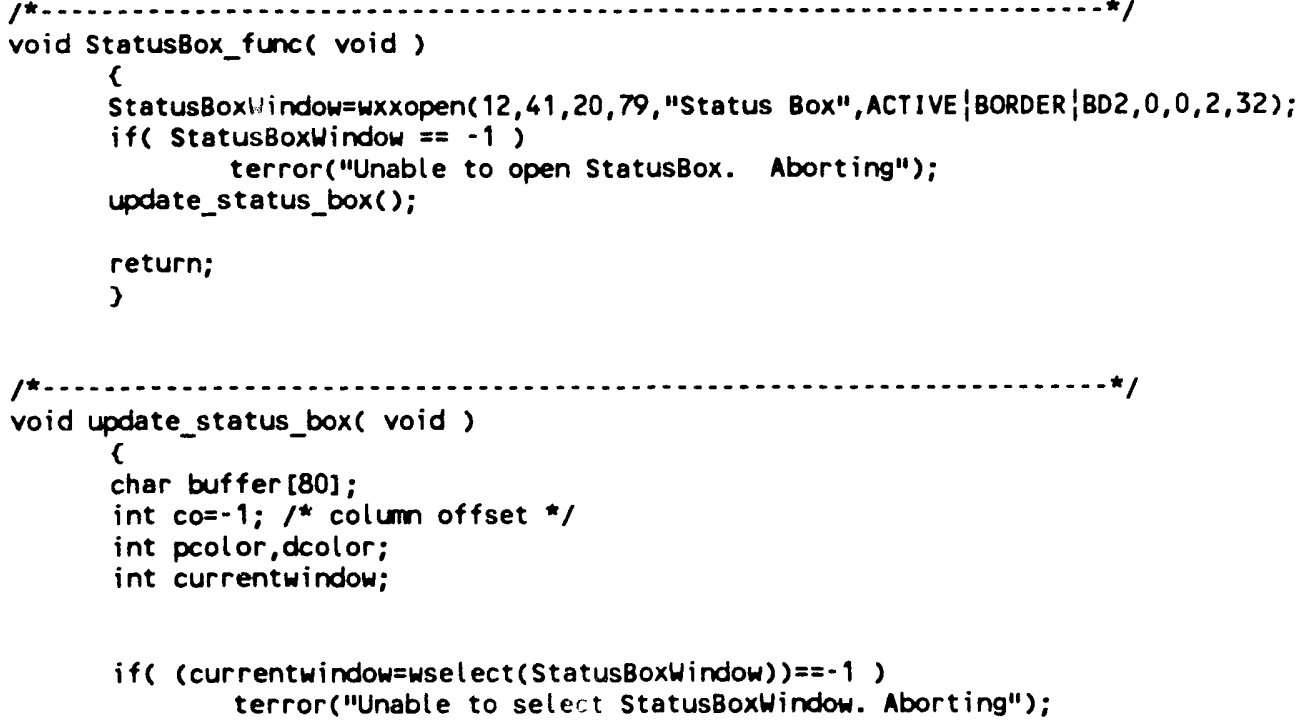




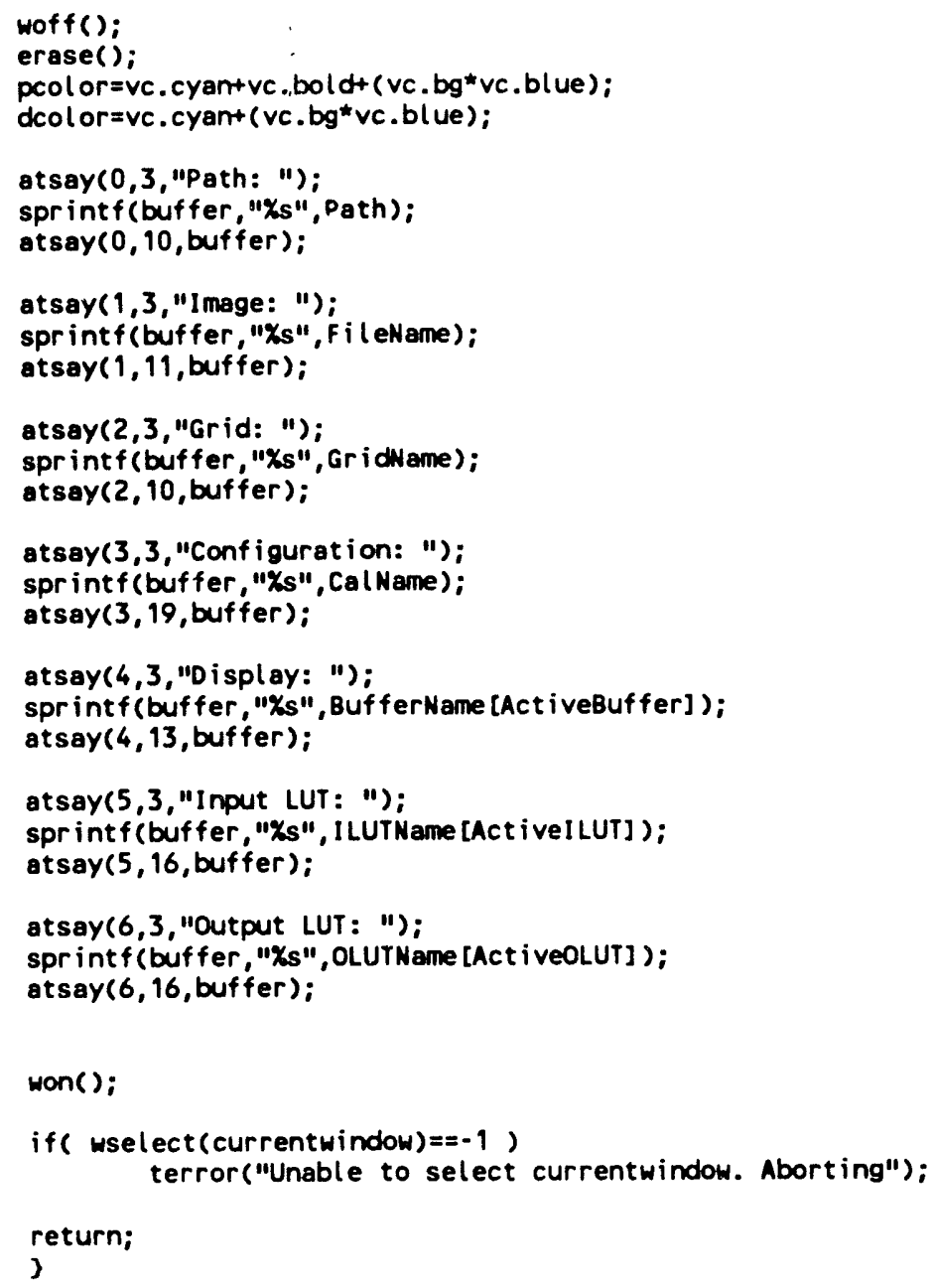

won();

if ( wselect (currentwindow) $==-1$ ) terror("Unable to select currentwindow. Aborting");

return;

3

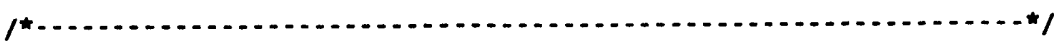

int caution( void)

int $i$;

int window;

char $y n=1 N$ ':

window $=w$ xopen $(15,0,23,40$, NULL , ACT IVE+CENTER , 1, 40);

atsay (1,3, "CAUTION - This function will delete");

atsay(2,3," the current buffer data");

atsay( $3,3, "$ for this option." );

atsay (5,3" Continue (Y/N) ? ... "1):

atget $(5,37,8 \mathrm{yn}, " \mathrm{~A} ")$;

readgets ():

wclose(window);

if( $y n==$ ' $N$ ') return(FAILURE); else return(SUCCESS);

)

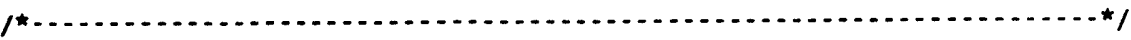

$1^{*} \mathrm{dt}$ cursor - The cursor function uses the DT2861 library calls to turn on the cursor in the active buffer and return its position and grey scale after user relocation. The arguments are: grey - pointer to the cursor grey value, row - pointer to the cursor row, col - pointer to the cursor colum, row_sensitivity - number of pixels to skip upon key stroke, col_sensitivity - number of pixels to skip upon key stroke.

* 


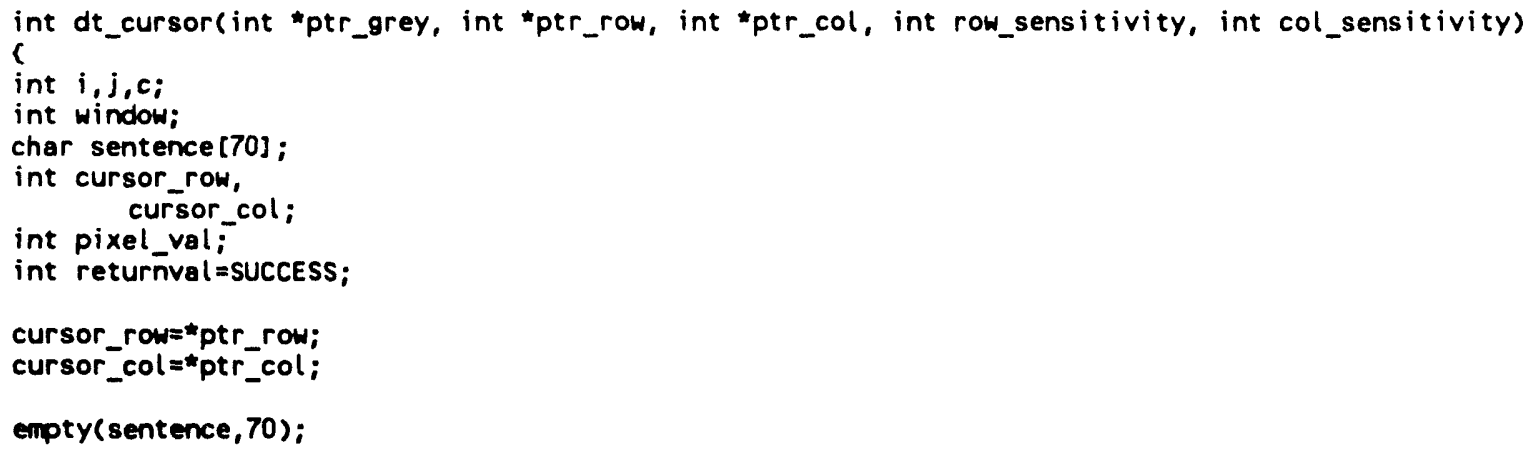

iff $c==$ DTUP ) cursor_row=cursor_row-row_sensitivity;

if( $c==0$ TDOWN ) cursör_row=cursör_rowtrow_sensitivity;

if ( $c==$ OTRIGHT) cursor_col=cursor_col+col_sensitivity;

if ( $c==$ DTLEFT ) cursor_col=cursor_col-col_sensitivity;

if( cursor_col>COL-1) cursor_col=cursor_col-col_sensitivity;

if( cursor_col<0) cursor_col=cursor_col+col_sensitivity;

if ( cursor_row>ROW-1 ) cursor_row=cursor_row-row_sensitivity;

if ( cursor_row<0) cursor_row=cursor_rowtrow_sensitivity;

IS_SET_CURSOR_POSITION(Cursor_rOW, cursor_col);

IS_GET_PIXEL(ĀctiveBuffer,cursor_row, cursor_col, 1,8pixel_val);

sprintf(sentence," Coordinate $=(x 3 d, 13 d)$

atsay $(1,0$, sentence);

cursor_row,cursor_col);

sprintf(sentence," Value $=$ x3d ", pixel_val);

atsay $(2,0$, sentence);

3

IS_CURSOR (OFF):

weTose(window);

\#ptr_grey=pixel_val;

-ptr row=cursor row:

*ptr_col=cursor_col;

return( SUCCESS ): 


\section{A-21}

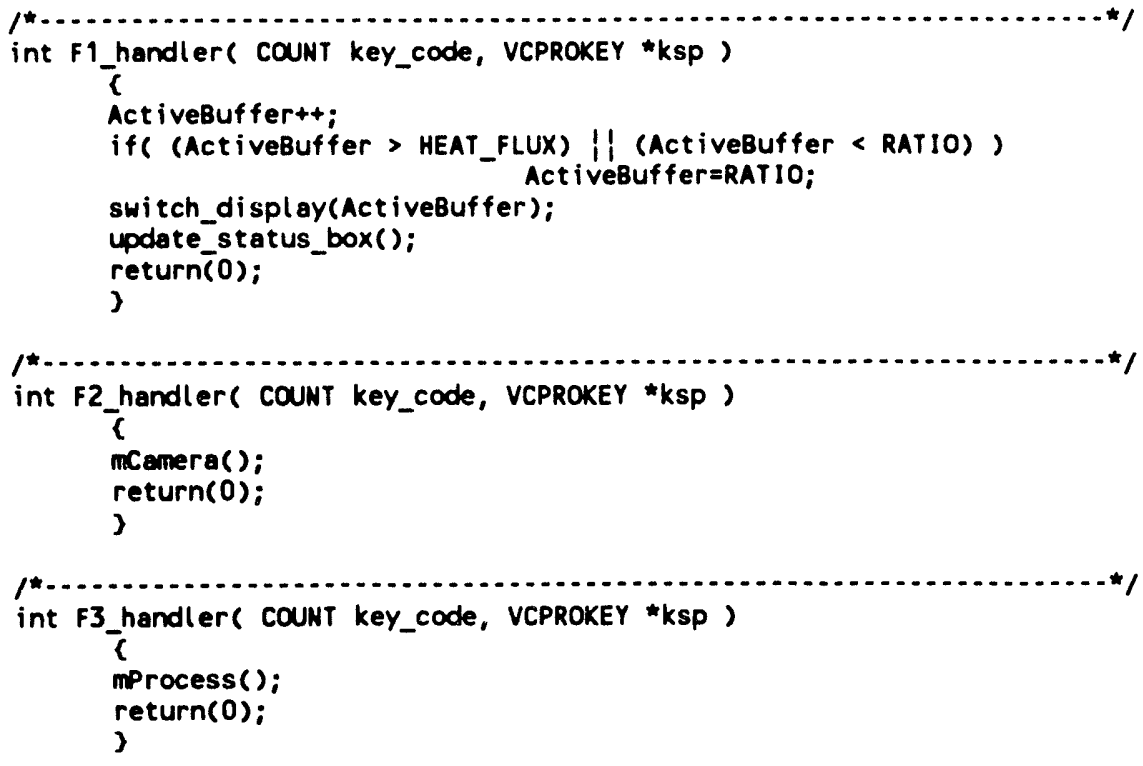




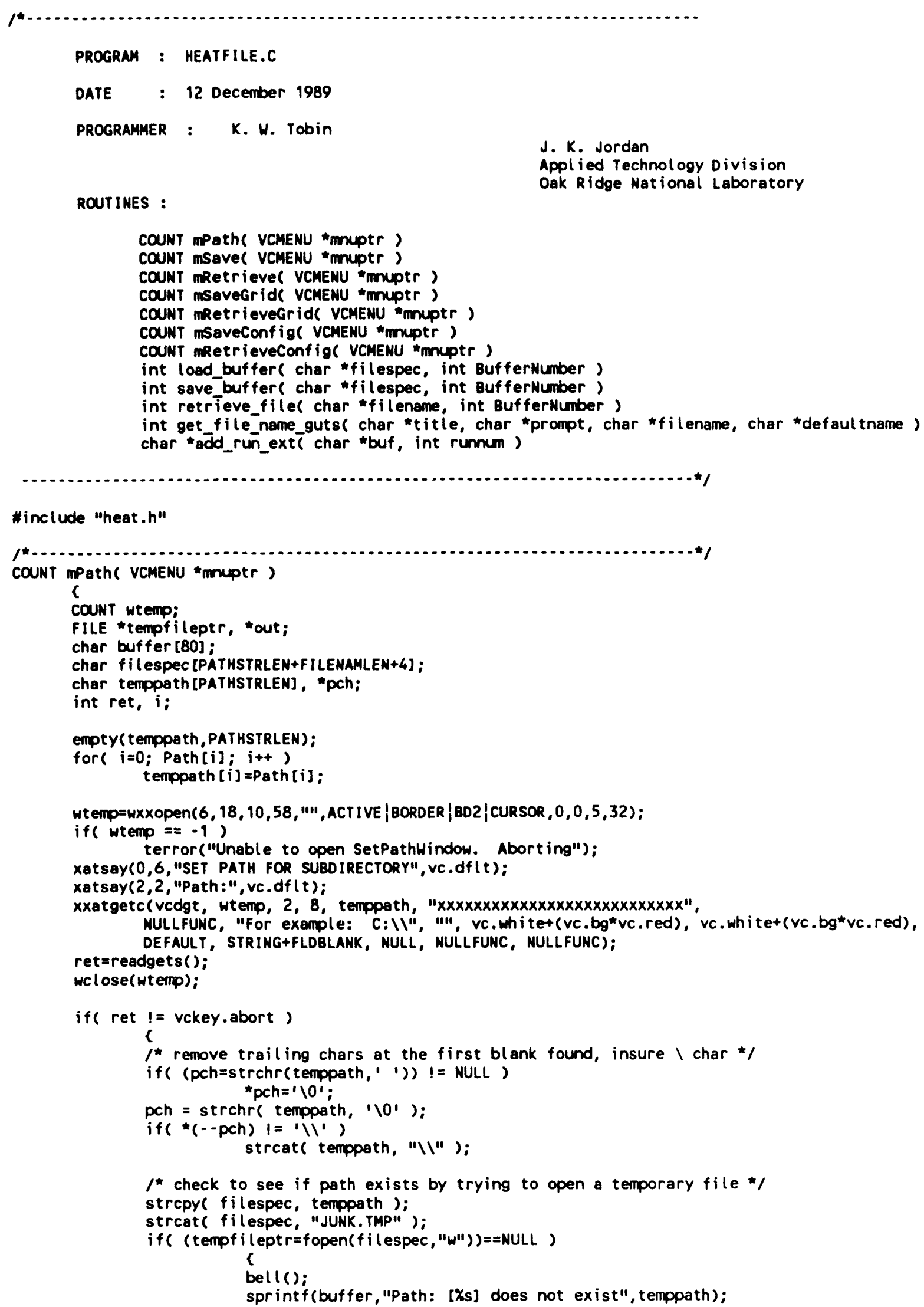

COUNT mPath ( VCMENU mmuptr )

COUNT mSave( VCMENU *muptr?

COUNT mRetrieve( VCMENU "monuptr )

COUNT mSaveGrids VCMENU "moptr )

COUNT mRetrieveGrid( VCMENU "muptr)

COUNT mSaveConfig( VCMENU *muptr)

COUNT mRetrieveConfig( VCMENU *mmuptr )

int load_buffer( char *ilespec, int BufferNumber)

int save buffer ( char *filespec, int BufferNumber)

int retrieve_file( char *filename, int BufferNumber)

int get_file_name_guts( char *title, char *prompt, char *filename, char *defaultname) char add_ruñexté( char *buf, int runnum)

\#include "heat.h" 


\section{A-23}

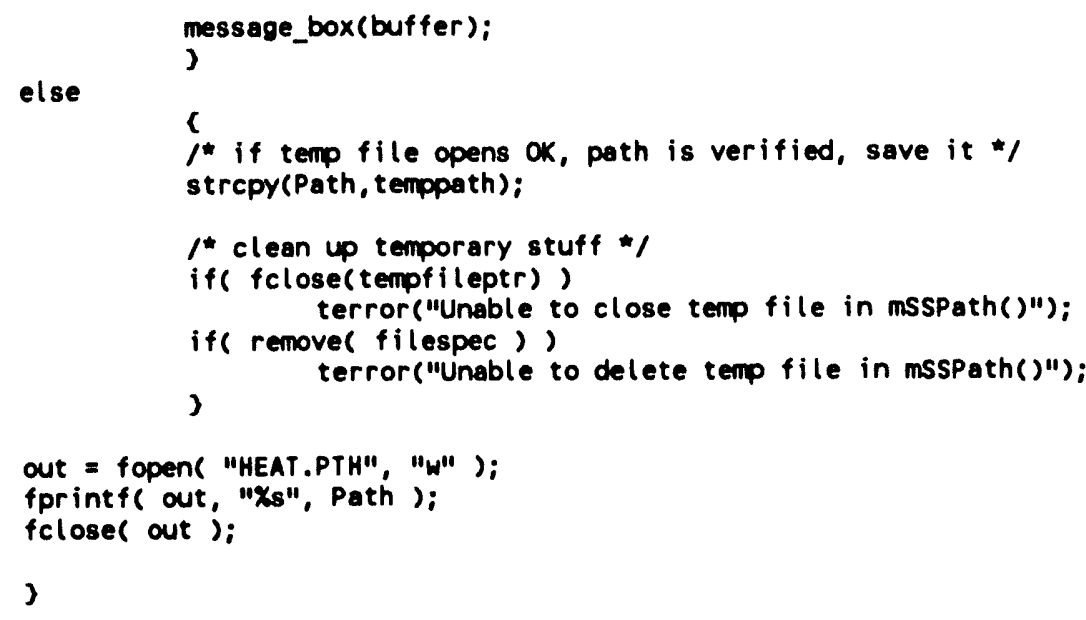




\section{c}

if( strlen( buf ) $>8$ ) $/ *$ do not exceded max filename size */ return NULL;

strcat ( buf, "." ): $/$ * 1.1 is not found, add extension */ strcat( buf, ext); 了

return buf:

3

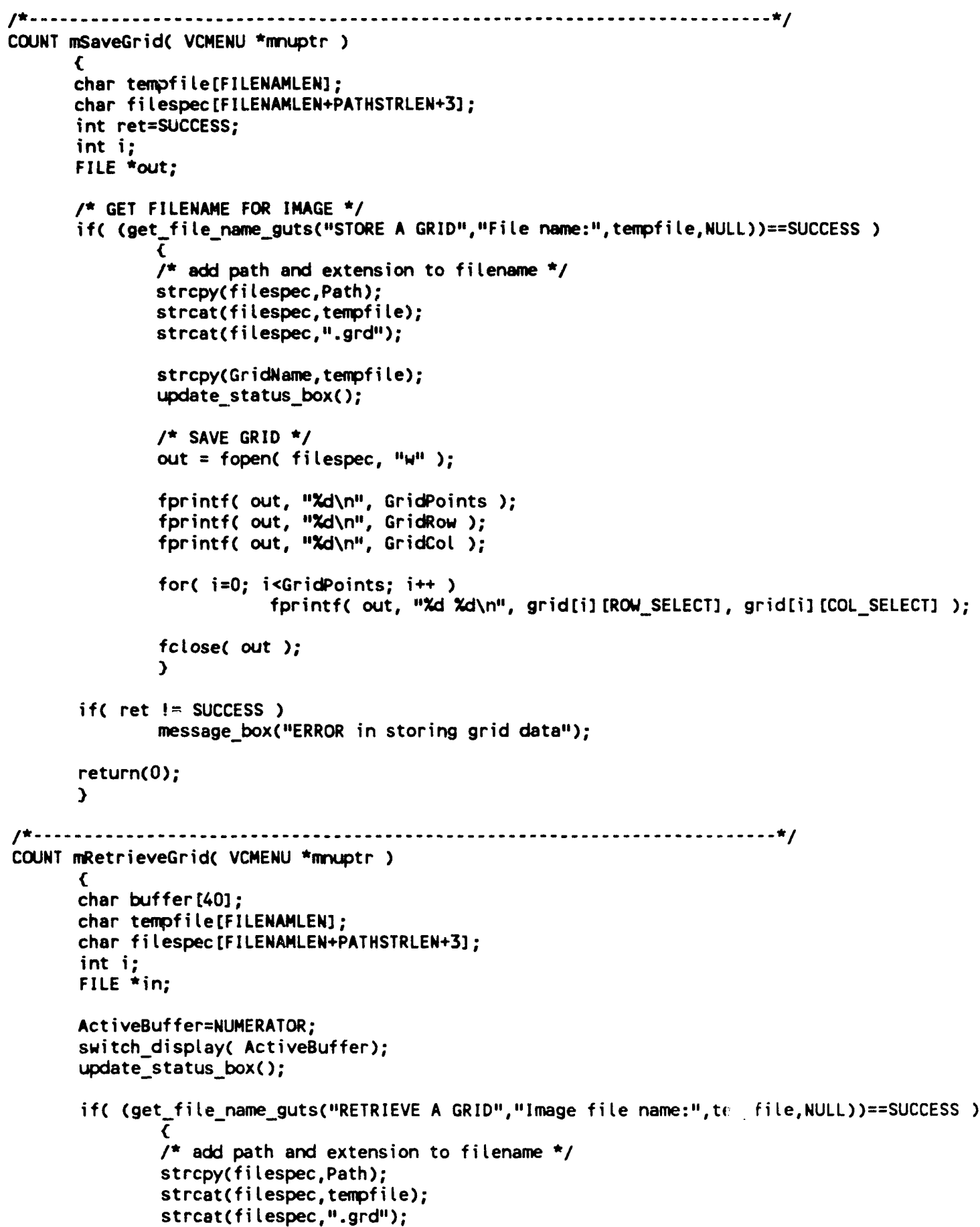

ACt iveBuffer=NUMERATOR;

switch_display( ActiveBuffer):

update_status_box();

if( (get_file_name_guts("RETRIEVE A GRID","Image file name:", te file,NULL))==SUCCESS ) c 


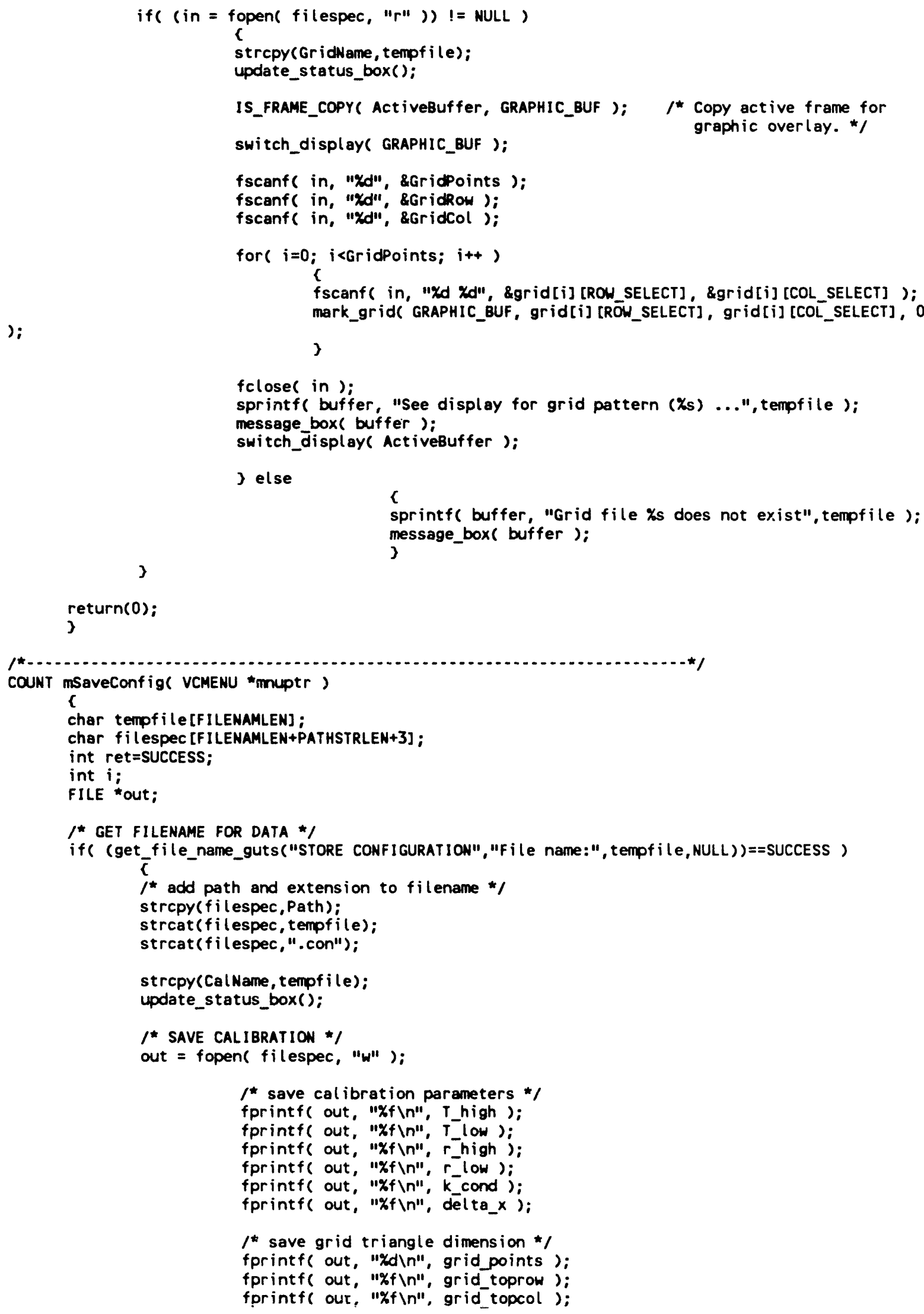

$\zeta$ sprintf( buffer, "Grid file \%s does not exist", tempfile); message_box( buffer);

了 了

return(0):

了

COUNT mSaveConfig( VCMENU "muptr)

\{

char tempfi le [FILENAMLEN] :

char fil espec [FILENAMLEN+PATHSTRLEN+3];

int ret=SUCCESS:

int $i$;

FILE *out;

I* GET FILENAME FOR DATA */ if (

$I^{*}$ add path and extension to filename */

strcpy ( $f i$ lespec, Path);

strcat ( $f i l$ espec, tempfile);

strcat (filespec,".con");

strcpy(Cal Name, tempfile);

update_status_box();

/* SAVE CALIBRation */

out $=$ fopen ( filespec, "w" ); 
fprintf( out, "Xfin", grid_botrow); fprintf( out, "Xfin", grid_botcol);

$I^{\star}$ save current path */

fclose( out ):

if ( ret ! = SUCCESS )

message_box("ERROR in storing calibration data");

return(0):

3

COUNT mRetrieveConfig( VCMENU *muptr)

c

char buffer [40];

char tempfile [FILENAMLEN];

char filespec [FILENAMLEN+PATHSTRLEN+3] ;

int $i$;

FILE *in;

if( (get file_name_guts ("RETRIEVE CONFIGURATION", "file name:", tempf $i$ le, NULL)) ==SUCCESS )

$$
\bar{c}
$$

i* add path and extension to filenani */

strcpy (filespec, Path):

strcat (filespec, tempfile);

strcat (filespec,".con");

if( (in = fopen ( filespec, "r" )) != NULL )

strcpy(Cal Name, tempfile);

update_status_box();

/* retreive calibration parameters */

fscanf( in, "Yf", \&T_high):

fscanf( in, "Xf", \&T (ow);

fscanf( in, "zf", \&r_high):

fscanf( in, "Yf", \&r_low );

fscanf( in, "Yf", \&k cond );

fscanf( in, "Xf", \&dé(ta_x);

/* retrieve grid triangle dimension */

fscanf( in, "Yd", \&grid_points):

fscanf( in, "Xf", \&grid toprow):

fscanf( in, "Yf", \&grid topcol):

fscanf( in, "Yf", \&grid_botrow):

fscanf( in, "Xf", \&grid_botcol);

1 * retrieve current path */

fclose (in):

J e!se

sprintf( buffer, "Calibration file Xs does not exist", tempfile); message_box( buffer):

了

)

return $(0)$;

) 


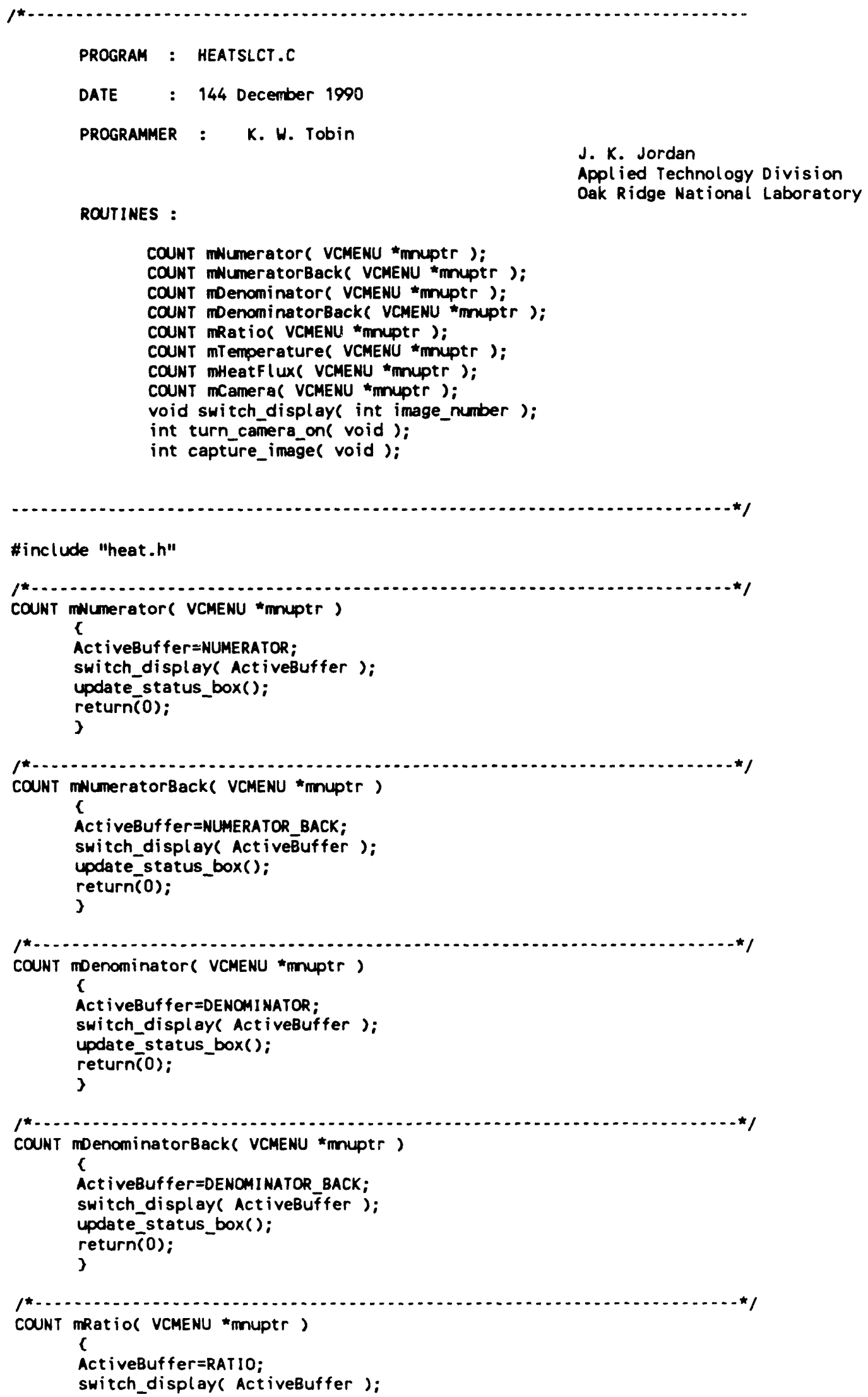

COUNT mNUmerator( VCMENU *mnuptr)

COUNT mNumeratorBack ( VCMENU *mnuptr):

COUNT mDenominator( VCMENU *muptr );

COUNT mDenominatorBack ( VCMENU *muptr);

COUNT mRatio( VCMENU *moptr):

COUNT mT emperature( VCMENU *mintr );

COUNT mHeatF (IUX( VCMENU "mouptr);

COUNT mCamera( VCMENU "mnuptr );

void switch_display( int image_number );

int turn camera_on( void);

int capture image( void ); 


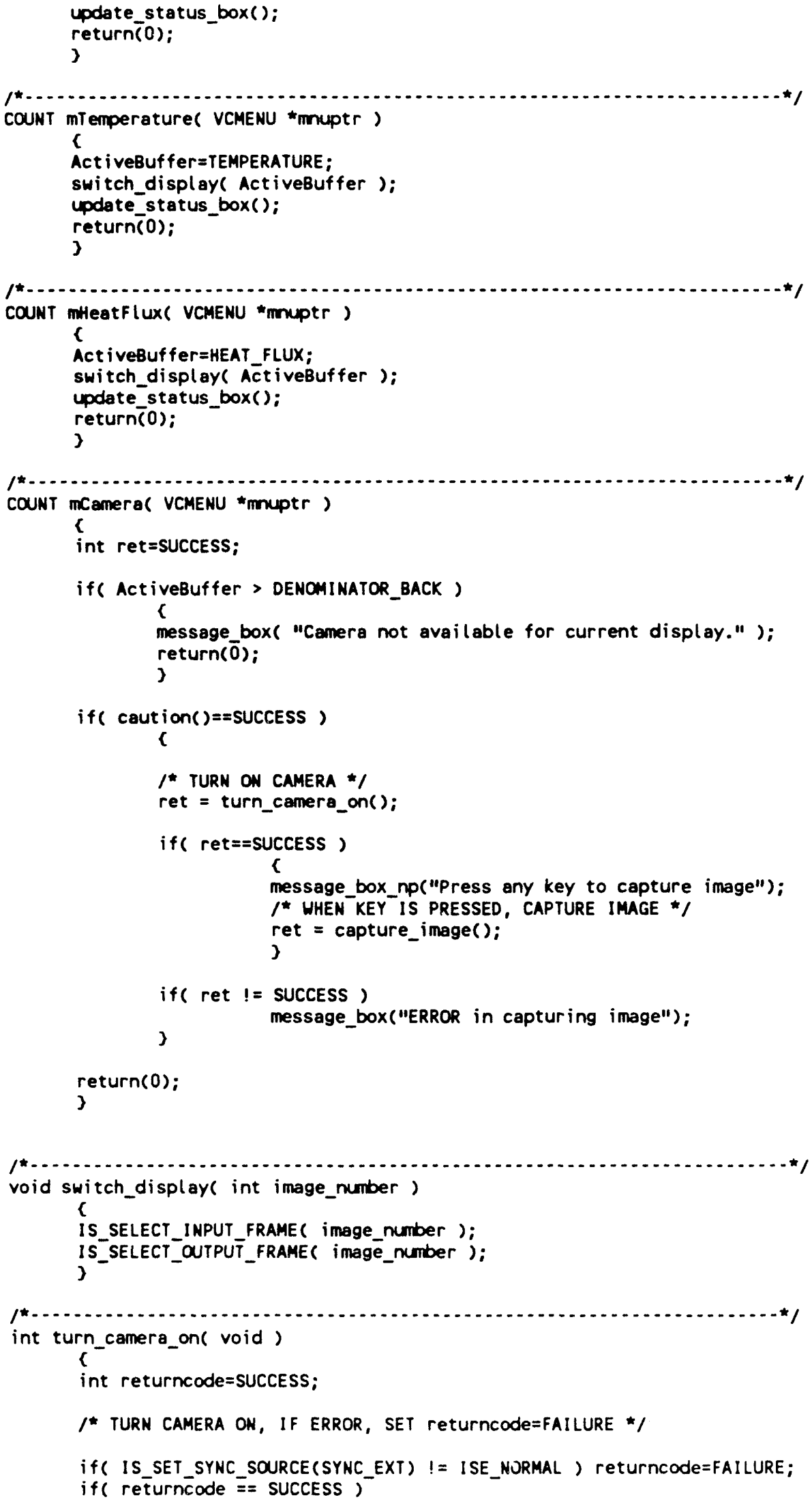


A-31

if( IS_PASSTHRU() I= ISE_NORMAL ) returncode=FAILURE;

return returncode;

了

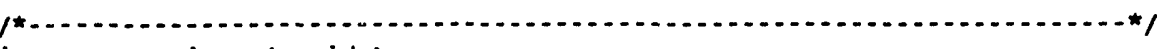

int capture_image( void)

c

int returncode=SUCCESS;

1* CAPTURe IMAGe here, IF ERROR, SET returncode=FAILURE */

IS_FREEZE_FRAME();

IS_ACOUIRE (ACtiveBuffer , 8); $\quad / *<-8$ frames averaged. */

IS_SET_SYNC_SOURCE(SYNC_INT);

return returncode;

了 


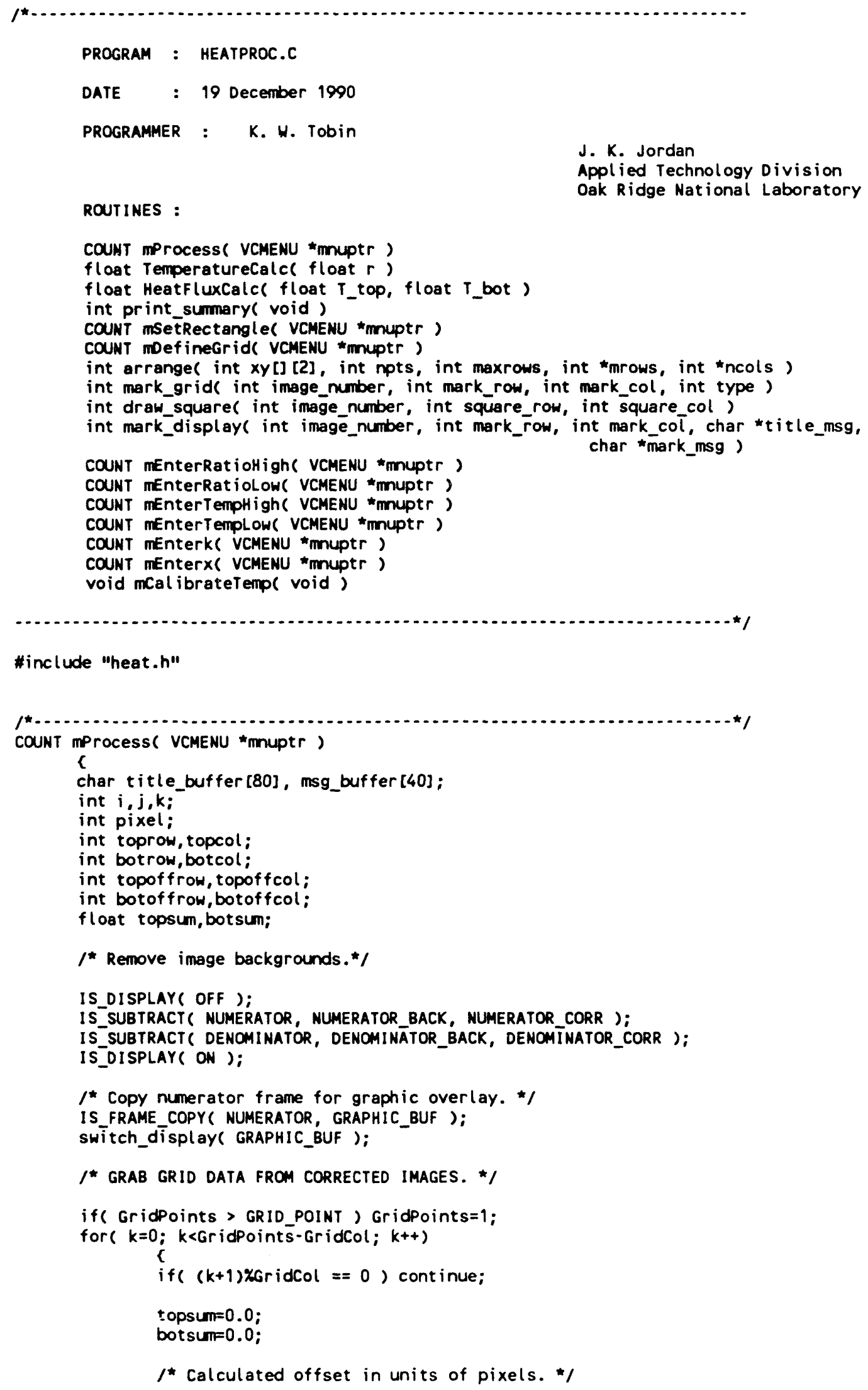




\section{A-33}

topof frow=grid toprow* (grid[k+GridCol] [ROW SELECT]-grid[k] [ROW SELECT]); topof fcol=grid_topcol * $(g r i d[k+1]$ [COL_SELECT̃] - grid [K] [COL_SELECT̃] ) ;

bot of frow=grid botrow* (grid[k+GridCol] [ROW SELECT] - grid[k] [ROW SELECT]); botoffcol=grid_botcol*(grid[k+1] [COL_SELECT̃]-grid[k] [COL_SELECT̄] );

/* Offset pixel coordinates for each phosphor cell. */ toprow=grid [K] [ROW_SELECT]+ topof frow;

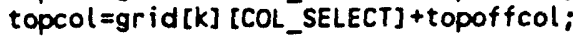

botrow $=g r i d[K]$ [ROW_SELECT]+bot of frow:

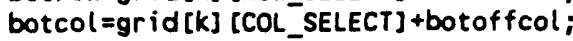

/* Mark image at offset location for visual verification. */ draw square( GRAPHIC BUF, toprow, topcol ); draw_square( GRAPHIC_BUF, botrow, botcol ):

$f^{*}$ Grab pixel data from PxP area for numerator phosphor. */ for ( $i=0$; $i<g r i d$ points; $i++$ )

C for $(j=0$; j<grid_points; $j++)$ < IS GET PIXELS NUMERATOR CORR, toprow-grid points $/ 2+i$

\&top_grey[K] [NUM_SELECT] ); topcol-grid_points/2+j, 1, topsunt=top_grey[K] [NUM_SELECT] ; IS_GET_PIXELC NUMERATOR_CORR, botrow-grid_points $/ 2+i$, botcol-grid_points/2+j, 1 ,

\&bot_grey[K] [NUM_SELECT] ); botsumt=bot_grey[K] [NUM_SELECT] ;

了 3 top_grey[k] [NUM_SELECT] $=($ int $)($ topsum/(grid_points*grid_points)); bot_grey $[k]$ [NUM_SELECT] $=($ int $)($ botsum/ (grid_points*grid_points));

topsum=0.0; botsum=0.0;

1* Grab pixel data from PxP area for denominator phosphor. */ for $i=0$; i<grid points; $i++$ )

c for $(j=0$; jegrid_points; $j++)$ IS_GET_PIXELC DENOMINATOR_CORR, toprow-grid_points/2+i

\&top_grey[K] [DEN_SELECT] ); topcol-grid_points $/ 2+j, 1$, topsunt =top_grey[K] [DEN_SELECT] ;

IS_GET_PIXEL( DENOMINATOR_CORR, botrow-grio_points $/ 2+i$, botcol-grid_points/2+j, 1 .

\&bot_grey[K] [DEN_SELECT] ); botsumt=bot_grey[K] [DEN_SELECT];

3 3

top grey [k] [DEN SELECI] $=($ int $)($ topsum/(grid points*grid points $)$ ); bot_grey $[K]\left[D E N \_S E L E C T\right)=($ int $)($ botsum/(grid_points*grid_points $\left.)\right)$;

if ( top_grey $[K]\left[D E N_{-}\right.$SELECT] $>0$ ) top_ratio $[k]=(f$ loat $)$ top_grey $[k][N U M$ SELECT $] /(f l o a t)$ top_grey $[K]$ [DEN_SELECT] ; top_temperature $[k]=$ TemperatureCalc ( top_ratio[k]);

J eTse

c top_ratio $[k]=0.0$;

top temperature $[k]=0.0$;

) 
if( bot_grey[K] [DEN_SELECT] > 0 )

c

bot ratio[k] $=(f$ loat $)$ bot grey[k] [NUM_SELECT] $/(f$ loat $)$ bot grey[k] [DEN_SELECT] ;

bot_temperature $[k]=$ TemperatureCalc( bot_ratio[k] );

J eise

bot_ratio[k] $=0.0$;

bot_temperature $[k]=0.0$;

heat_flux $[k]=$ HeatF luxCalc( top_temperature $[k]$ ，bot_temperature $[k]$ );

)

/* DISPLAY RATIO DATA ON IMAGE BUfFER */

IS_FRAME_COPY( MUMERATOR, RATIO);

ActiveBǘf fer=RATIO;

switch_display( ActiveBuffer );

update_status_box():

sprintf( title_buffer, "PHOSPHOR RATIO DATA (front/back)" );

for ( $k=0$; $k<$ Gridpoints-GridCol; $k++$ )

if( $(k+1)$ xaridcol $==0)$ continue;

sprintf( msg_buffer, "x3.2f", top_ratio[k] );

mark_displaye Act iveBuffer, gridtk] [ROW_SELECT], grid[k] [COL_SELECT], title_buffer, msg_buffer);

sprintf( msg_buffer, "x3.2f", bot_ratio[k] );

mark_display( ActiveBuffer, grid[K] [ROW_SELECT]+17, grid[k] [COL_SELECT]+5, title_buffer , 3 msg_buffer );

/* DISPLAY TEMPERATURE daTA ON IMAGe BUfFER */

IS FRAME COPY ( NUMERATOR, TEMPERATURE ):

ACT $\bar{T}$ iveBuff $\mathrm{Ser}=$ TEMPERATURE:

switch_display( ActiveBuffer);

update_status_box();

sprintfe title buffer, "PHOSPHOR TEMPERATURE DATA ( $\left.\psi_{s}\right) "$, DTTUNIT);

for ( $k=0$; $k<G$ ridpoints-GridCol; $k++$ )

c

if $(k+1)$ zoridcol $==0)$ continue;

sprintf( msg_buffer, "W4.1f", top_temperature[k] );

mark_display, Act iveBuffer, grid[k] [ROW_SELECT], grid[k] [COL_SELECT], title_buffer, msg_buffer);

sprintf( msg buffer, "Y4.1f", bot temperature[k] );

mark_display( ActiveBuffer, grid[k] [ROW_SELECT]+17, grid[k] [COL_SELECT]+5, title_buffer , 3 ms.'_buffer);

1 * Display heat flux data on Image buffer */

IS_FRAME_COPY( NUMERATOR, HEAT_FLUX);

ACË iveBUf̆fer=HEAT FLUX;

switch_display( Ac̄tiveBuffer);

update_status_box();

sprintf( title_buffer, "PHOSPHOR HEAT FLUX DATA ( $\left.\chi_{s}\right) "$ ", DTQUNIT);

for ( $k=0$; $k<$ GridPoints-GridCol; $k++$ )

iff $(k+1) x a r i d c o l==0)$ cont inue; 


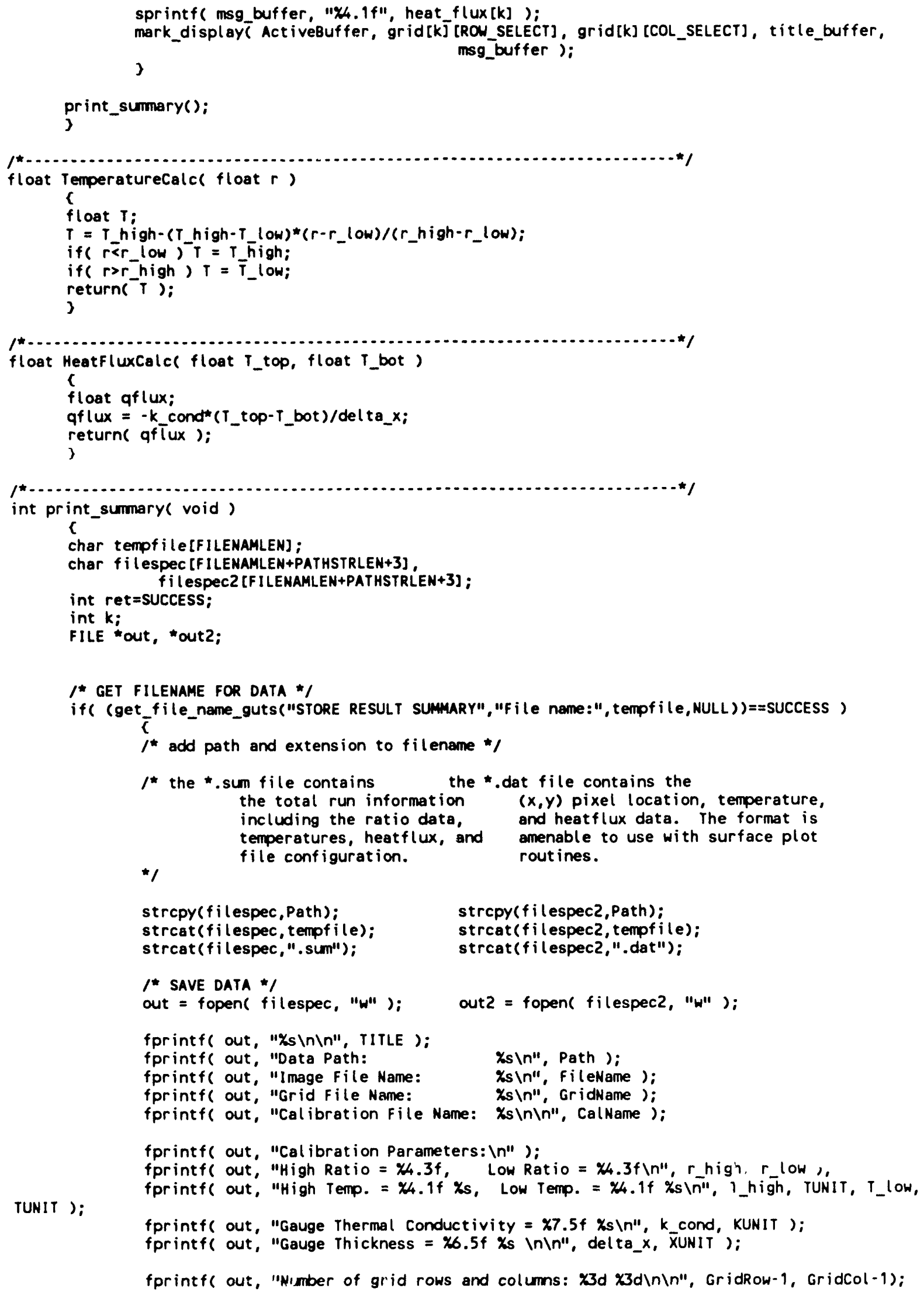


fprintfe out, "Data: ( $x, y$, front ratio, back ratio, front temp, back temp., heat $f(u x) \backslash n$ "):

for $k=0$; $k<$ GridPoints-GridCol; $k++)$

C

if( $(k+1) \times g r i d C o l==0$ ) cont inue;

fprintfe out, "X3d X3d $\times 5.4 f \times 5.4 f \times 5.2 f \times 5.2 f \times 6.2 f \backslash n "$, grid[k] [0], grid [k] [1], top_ratio[k],bot_ratio[k], top_temperature $[k]$, bot_temperature $[k]$, heat_flux [k] ; ;

fprintfl out2, "x3d $\times 3 d \times 5.2 f \times 5.2 f \times 6.2 f \backslash n ", g r i d[k][0], g r i d[k][1]$, 3 top_temperature $[k]$, bot_temperature [k], heat_f(ux [k]);

fclose( out );

fclose (out2):

)

if ( ret $!=$ success )

message_box ("ERROR in storing process data");

return(0):

3

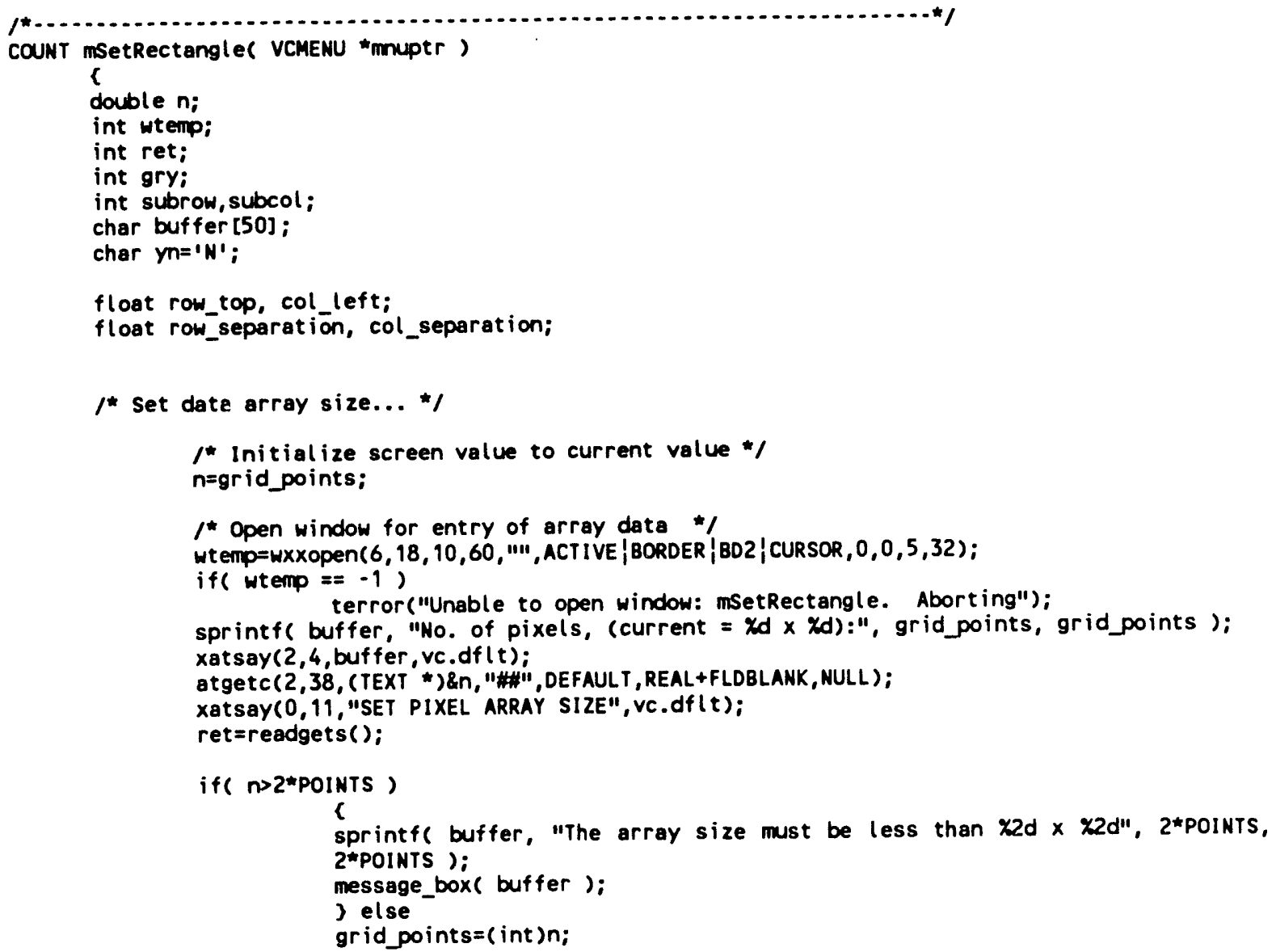


terror("Unable to open window: mSetRectangle. Aborting"); xatsay(0,11, "SET RECTANGLE COORDINATES",ve.df $t)$;

subrow=ROW/2;
subcol $=$ COL $/ 2$;

xatsay(2,3, "Set upper left of rectangle,",vc.dflt);

dt_cursor( \&gry, \&subrow, \&subcol, 2, 2);

row_top=subrow;

col_left=subcol:

mark_grid( GRAPHIC_BUF, subrow, subcol, 0 ):

xatsay(3,3, "Set upper left of rectangle below,",vc.dflt):

dt_cursor( \&gry, \&subrow, \&subcol, 2, 2 );

row_separation=subrow-row_top; mark_grid( GRAPHIC_BUF, subrow, subcol, 0 );

xatsay(4,3, "Set upper left of rectangle to right,",vc.df(t); dt cursor ( \&gry, \&subrow, \&subcol, 2, 2 );

col_separation=subcol-col_left; mark_grid( GRAPHIC_BUF, subrow, subcol, 0 );

xatsay(5,3, "Set lower triangle of main rectangle,",vc.df $(t)$; dt cursor( \&gry, \&subrow, \&subcol, 2, 2 ); grid toprow= ( subrow-row top)/row separation:

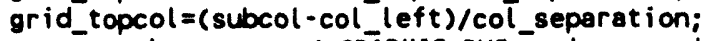
draw square( GRÄPHIC_BUf, subrow, subcol);

xatsay(6,3, "Set upper triangle of main rectangle.",vc.df(t); dt_cursor ( \&gry, \&subrow, \&subcol, 2, 2 ); grid_botrow=(subrow-row_top)/row_separation: grid_botcol = (subcol-col-left)/col_separation; dran_square( GRÄPHIC_BUF, subrow, subcol );

wclose(wtemp);

message box ( "Remember to update the current configuration file!"); switch_display (ActiveBuffer);

return(0):

COUNT mDefineGrid( VCMENU *mnuptr )

c

int window;

int $i, j, k, i$;

int grid row $=50$. grid_col $=50$;

int maxrows $=25$;

char sentence [70] :

/* Initialized to place first cursor. */

/* Used by arrange() for sort routine. */

ActiveBuffer=NUMERATOR;

switch display (ActiveBuffer);

update_status_box();

window=wxxopen $(8,10,14,60$, NULL, ACT IVE+CENTER+CURSOR, 1, 40,5,32);

atsay (1,1, "Press <ENTER> to introduce new grid point,");

atsay (2,1,"Press 〈ESC> to quit ...");

IS_FRAME_COPY( ActiveBuffer, GRAPHIC_BUF ); /* Copy active frame for graphic overlay. */ switch_dísplay( GRAPHIC_BUF );

GridPoints=GRID_POINT;

$\operatorname{for}\left(k=0 ; k<G R I D \_P O I N T ; k++\right)$

sprintf( sentence, "Move cursor to point $x 3 \mathrm{~d}$ of $\times 3 \mathrm{~d} ", k+1$, GRID_POINT ); atsay $(4,1$, sentence); 


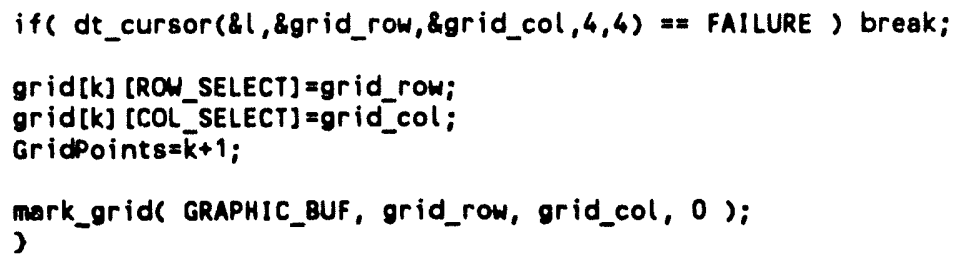

Function arrange(): given a list of coordinate pairs in arbitrary order, orrange them logically into a 2-d grid, determine the number of rows and columns, and sort the list by row and colum.

\section{J.K.Jordan $1 / 22 / 90$}

input:

$x y[][2]$ : the list of coordinates. $x y[][0]$ is the $x$ (row) and $x y$ [] [1] is the $y$ (column)

npts: the number of coordinate pairs in the list

maxrows: the maximum number of rows possible, used to determine the minimum possible row height

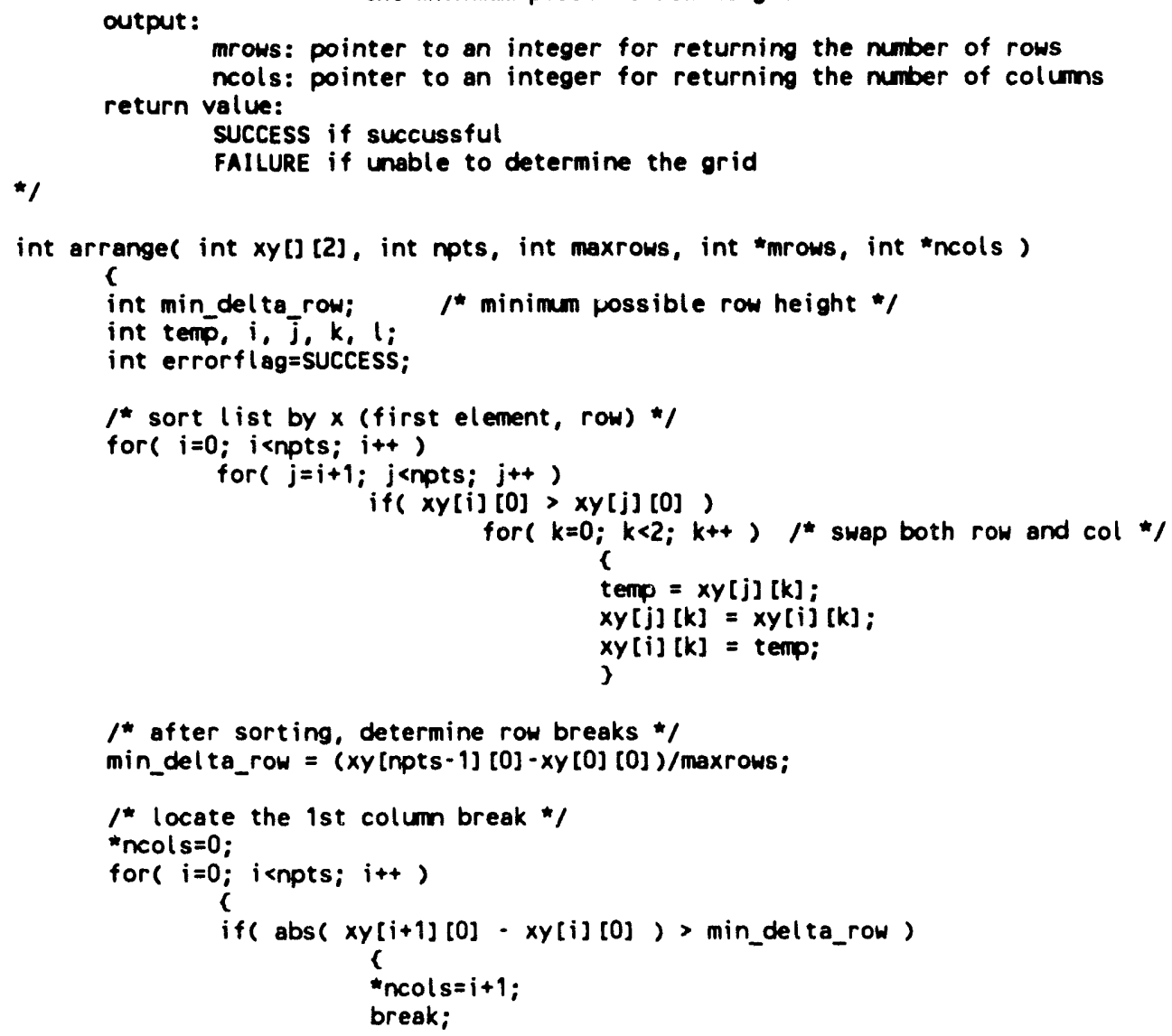


"mrows=npts/("ncols);

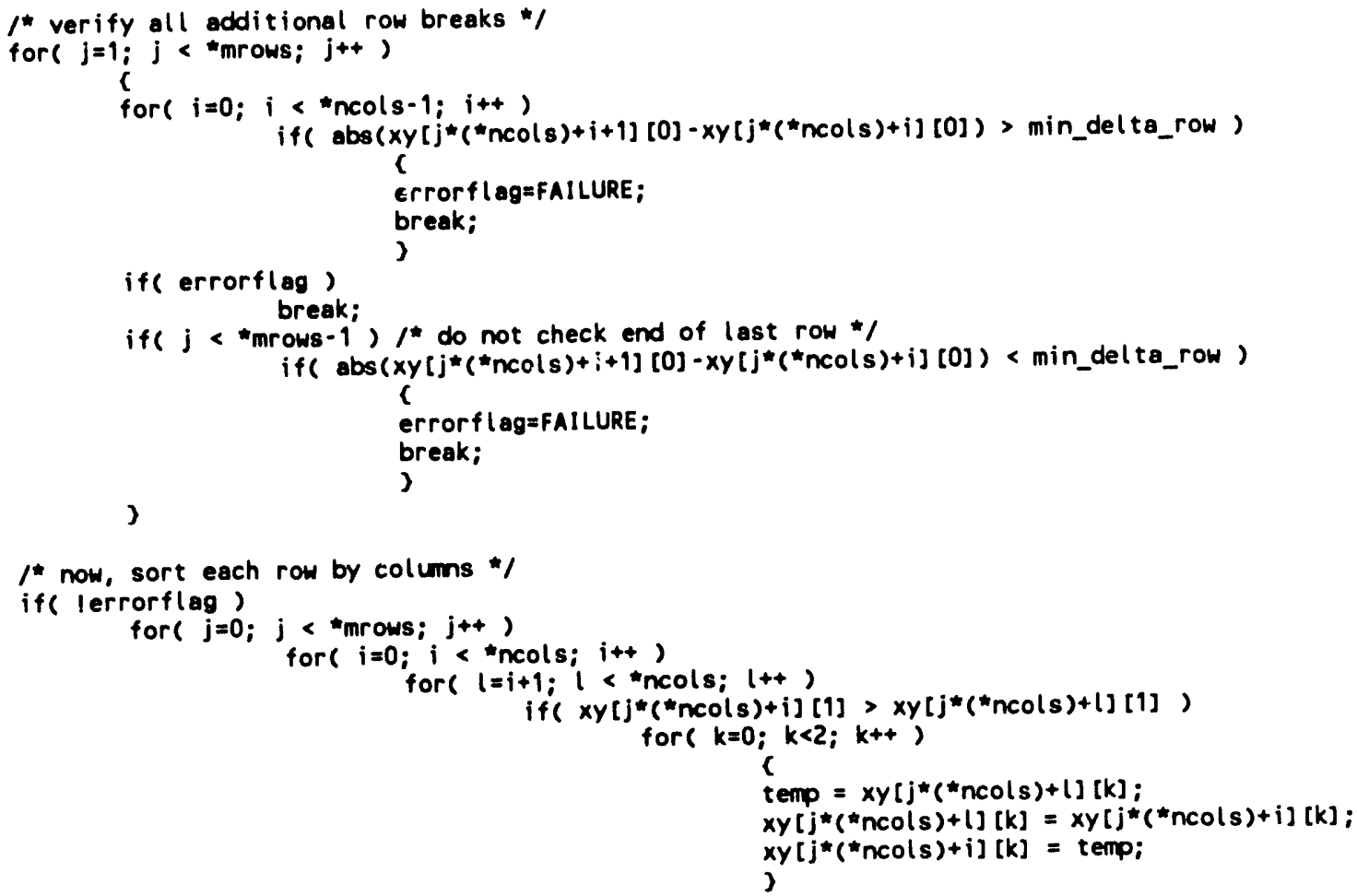

return( errorflag ):

)

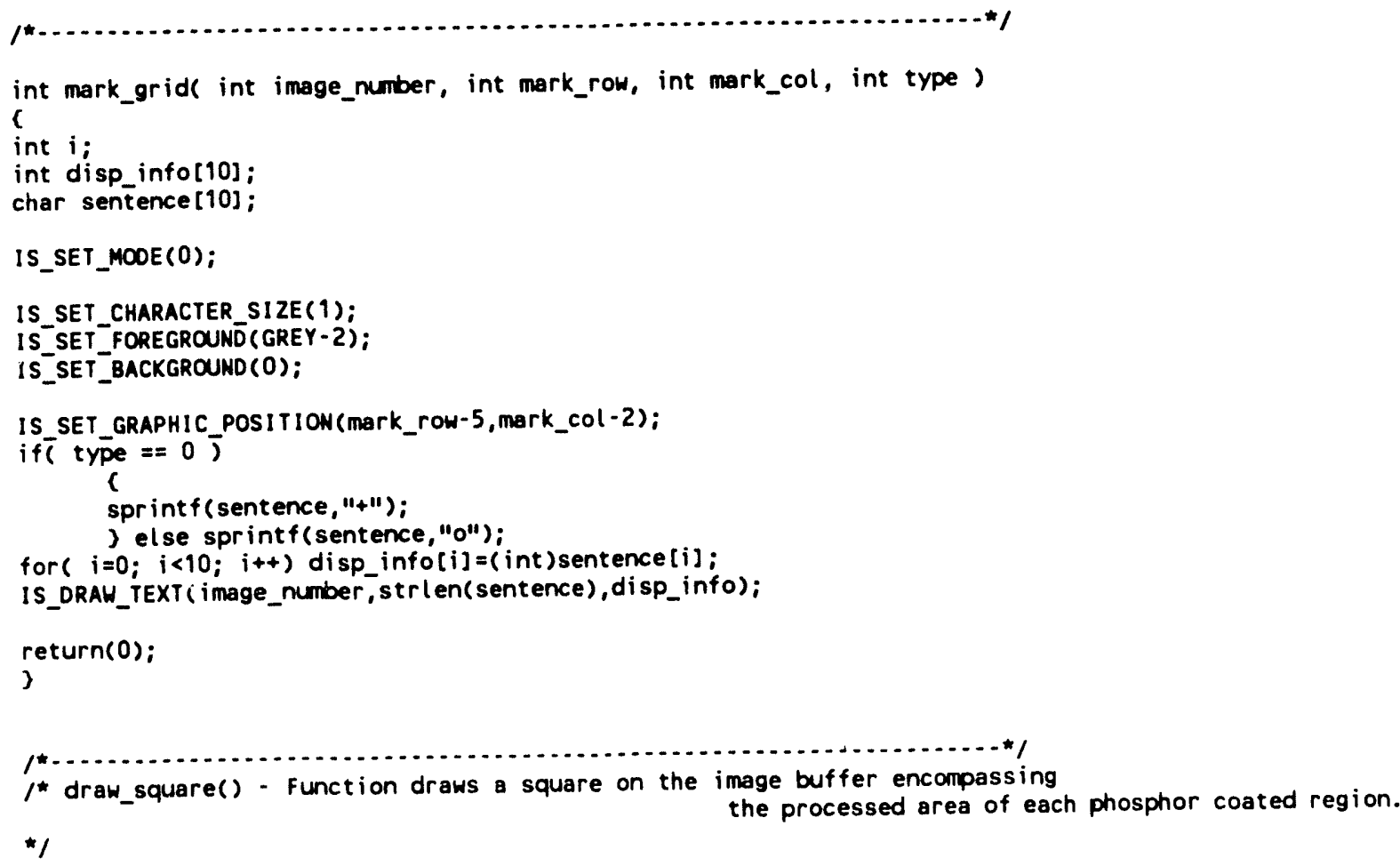


int draw_square( int image_number, int square_row, int square_col)

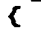

int point_count $=4$;

int point_array[4] [2] :

point_array $[0][0]=s q u a r e \_r o w+0 ; \quad$ point_array $[0][1]=s q u a r e \_c o l+$ grid_points;

point array [1] [0] =square row + grid points ; point array [1] [1] =square col + grid points;

point_array [2] [0] =square_row + grid_poirits; point_arrey[2] [1] =square_col + 0;

point_array $[3][0]=$ square_row;

point_array $[3][1]=$ square_col;

IS_SET_MODE $(0)$;

IS SET FOREGROUND(GREY-2);

IS_SET_BACKGROUND(0);

IS_SET_GRAPHIC_POSITION( square_row, square_col );

IS_DRAT̄_LINES( image_number, point_count, point_array);

)

1*

$1^{*}$ mark display() - Function will write msgo to the top line of the RGB

*1

display, msg1 will be written at coordinate (mark_row,mark_col).

int mark_displaye int image_number, int mark_row, int mark_col, char *title_msg,

c

int $i$;

int disp_info[40]:

char sentence $[40]$;

IS_SET_MOOE(O);

IS_SET_CHARACTER_SIZE(1);

IS_SET_FOREGROUND (GREY-2);

IS_SET_BACKGROUND(0);

IS SET GRAPHIC POSITION $(30,50)$;

sprint $\bar{f}($ sentence, title msg);

for $(i=0 ; i<40 ; i++)$ disp_ info[i]=(int) sentence $[i]$;

IS_DRAH_TEXT (image_number,strlen(sentence), disp_info);

IS_SET_GRAPHIC_POSITION(mark_rOW-5, mark_col-2);

sprintfo ( sentence, mark msg );

for $(i=0 ; i<40 ; i++)$ disp_info[i]=(int)sentence $[i]$;

IS_DRAW_TEXT (image_number,strlen(sentence),disp_info);

return(0);

了

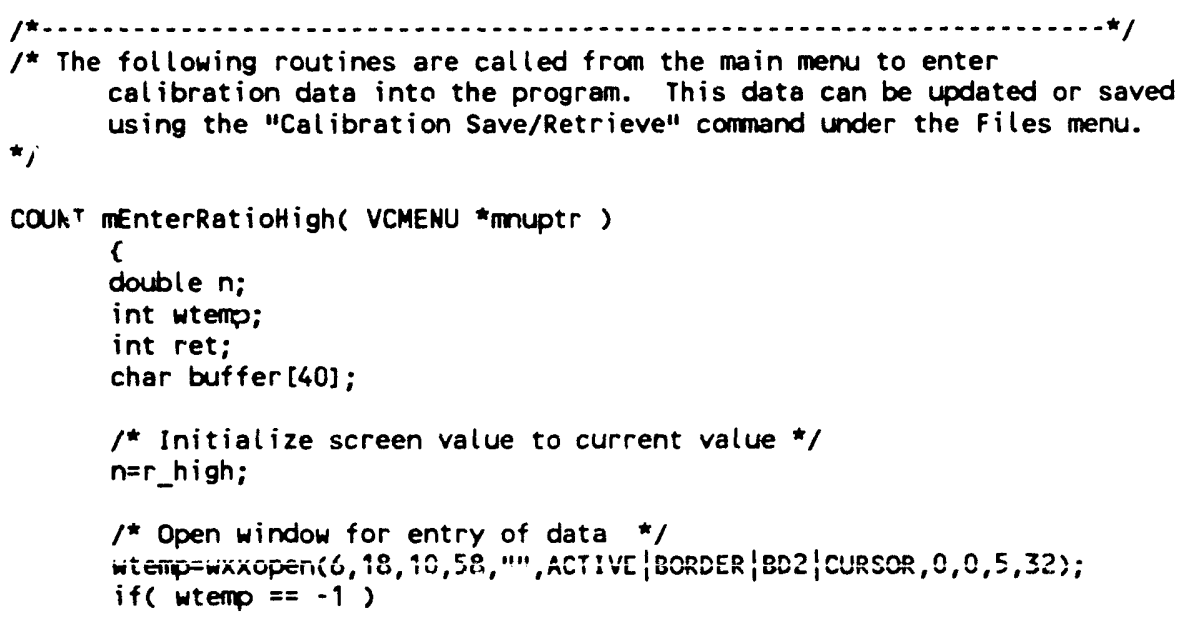


terror("Unable to open window: mEnterRatioHigh. Aborting"); xatsay $(2,4$, "New high ratio value:",vc.df $(t)$ :

atgetc $(2,30$, (TEXT *)\&n,"\#\# .\#",DEFAULT, REAL+FLDBLANK, NULL);

xatsay(0,11, "SET HIGH RATIO",vc.df $(t)$;

ret=readgets () ;

wclose(wtemp);

r_high=n;

return $(0)$

3

(*

COUNT mEnterRatioLow( VCMENU "mouptr )

double $n$;

int wtemp;

int ret;

char buffer [40];

$f^{*}$ Initialize screen value to current value */

$n=r_{-}$low;

I* Open window for entry of data *I

wtemp=wXXopen $(6,18,10,58, " 1 "$, ACT IVE |BORDER |BD2 |CURSOR , 0, 0,5,32).

if (wtemp $==-1$ )

terror("Unable to open window: menterRatiolow. Aborting");

xatsay $(2,4$, "New low ratio value:", vc.dflt $t$ ):

atgetc (2,30, (TEXT *)\&n, "\#. . DE", DEFAULT, REAL+FLDBLANK, NULL):

xatsay(0,11, "SET LOW RATIO",vc.df $(t)$;

ret $=$ readgets () .

welose (wtemp);

if( $n>r_{-}$high )

sprintf( buffer, "The low ratio must be less than $\times 6.4 \mathrm{f."}, r_{-}$high ); message_box( buffer):

J else

r_low=n;

return( $(0)$

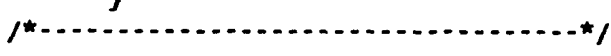

COUNT mEnterTempHigh( VCMENU *mouptr )

r

double $n$;

int Htemp;

int ret;

char buffer [40];

/* Initialize screen value to current value */

n=T_high;

* Open window for entry of data */

wt emp=wxxopen $(6,18,10,58$,"'", ACTIVE |BORDER |BD 2 |CURSOR , 0, 0, 5, 32);

if ( wtemp $==-1$ )

terror("Unable to open window: menterTempHigh. Aborting");

sprintf( buffer, "High temp. value (Zs):", TUNIT);

xatsay $(2,4$, buffer, vc.df $(t)$;

atgetc (2,30,(TEXT *)\&n, "\#\#" \#",DEFAULT, REAL+FLDBLANK, NULL);

xatsay $(0,11$,"SET HIGH TEMPERATURE",vc.df $(t)$;

ret=readgets ()

wclose (wtemp);

T_high=n;

return( 0 ): 


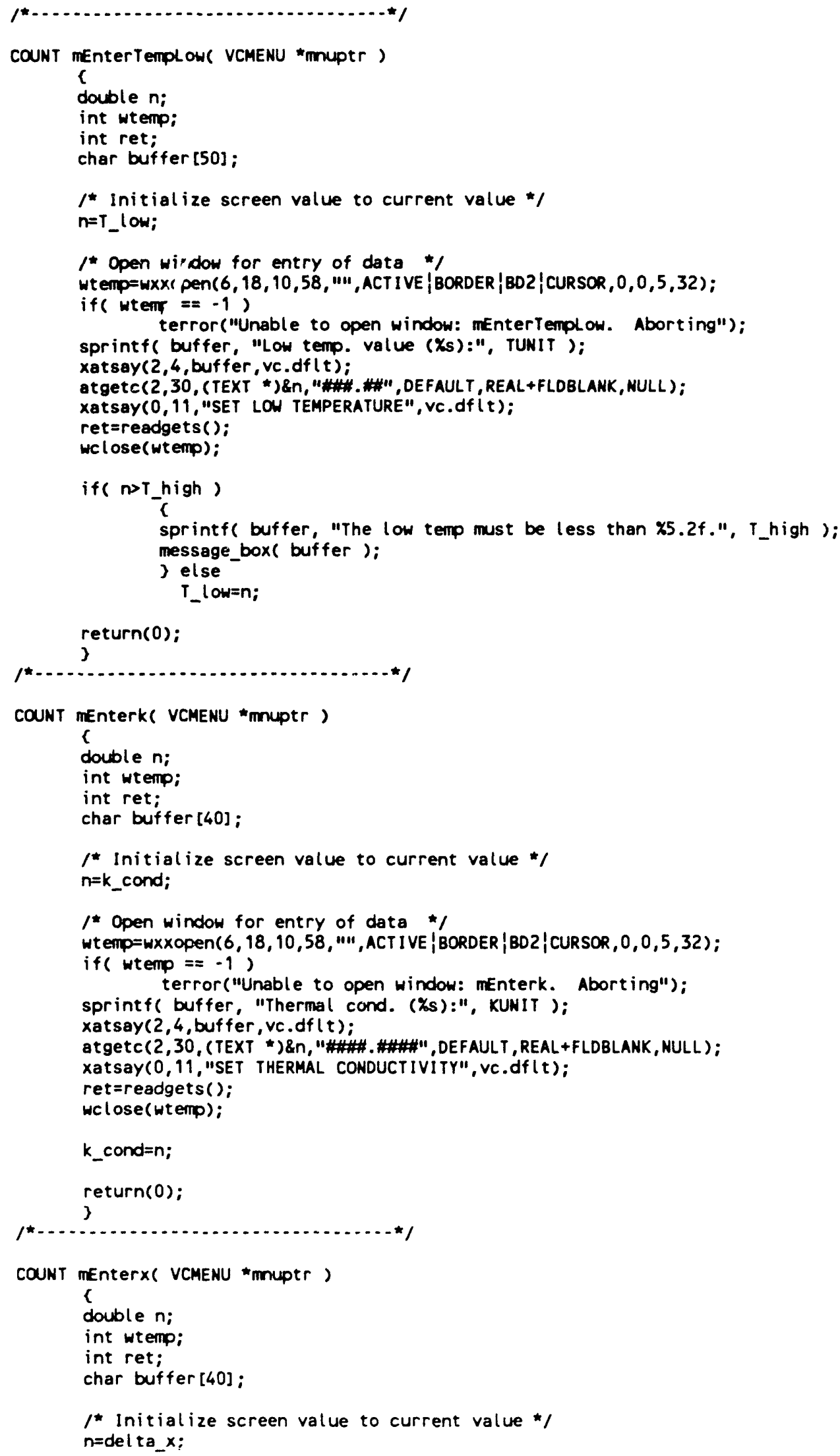


1 * Open window for entry of data */

wt emp=wxxopen $(6,18,10,58, " 1 "$, ACT IVE |BORDER |BD2 (CURSOR , 0, 0, 5, 32);

if ( wtemp $==-1$ )

terror("Unable to open window: mEnterx. Aborting");

sprintf( buffer, "Gauge thickness ( $\left.\chi_{s}\right): "$, XUNIT ):

xatsay $(2,4$, buffer, vc.df $(t)$;

atgetc $(2,30,($ TEXT *)\&n, "\#\#" DEFAULT, REAL+FLDBLANK, NULL);

xatsay $(0,11$, "SET GAUGE THICKNESS",vc.df $(t)$;

ret $=$ readgets () ;

wclose(wtemp);

delta_x=n;

return(0):

)

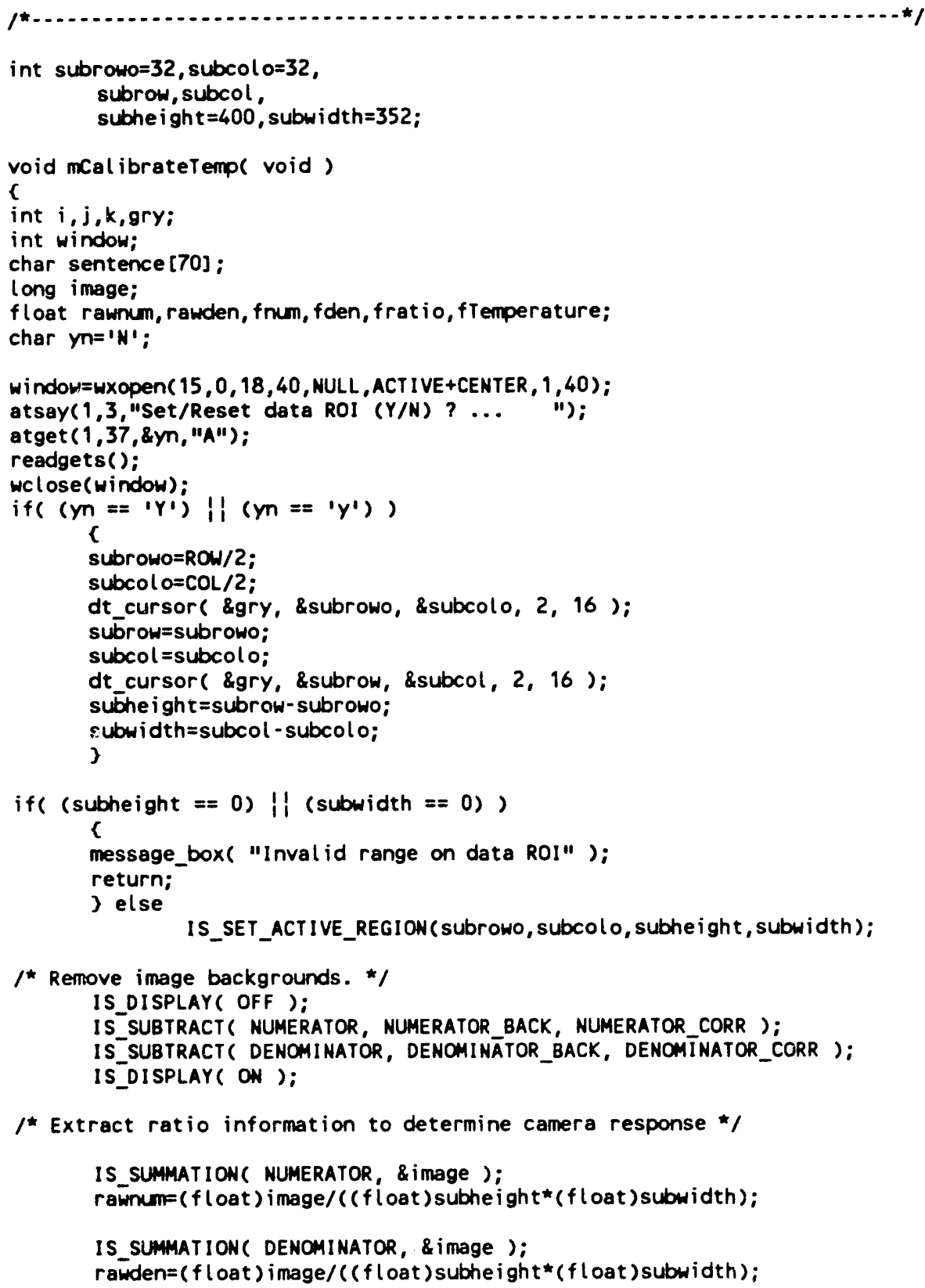

1 * Extract ratio information to determine camera response */

IS SUMMATION( NUMERATOR, \&image);

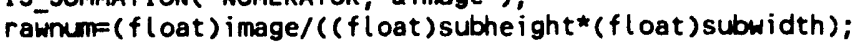

IS_SUMMATION( DENOMINATOR, \&image);

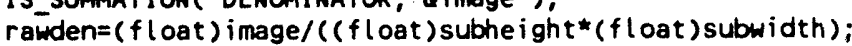




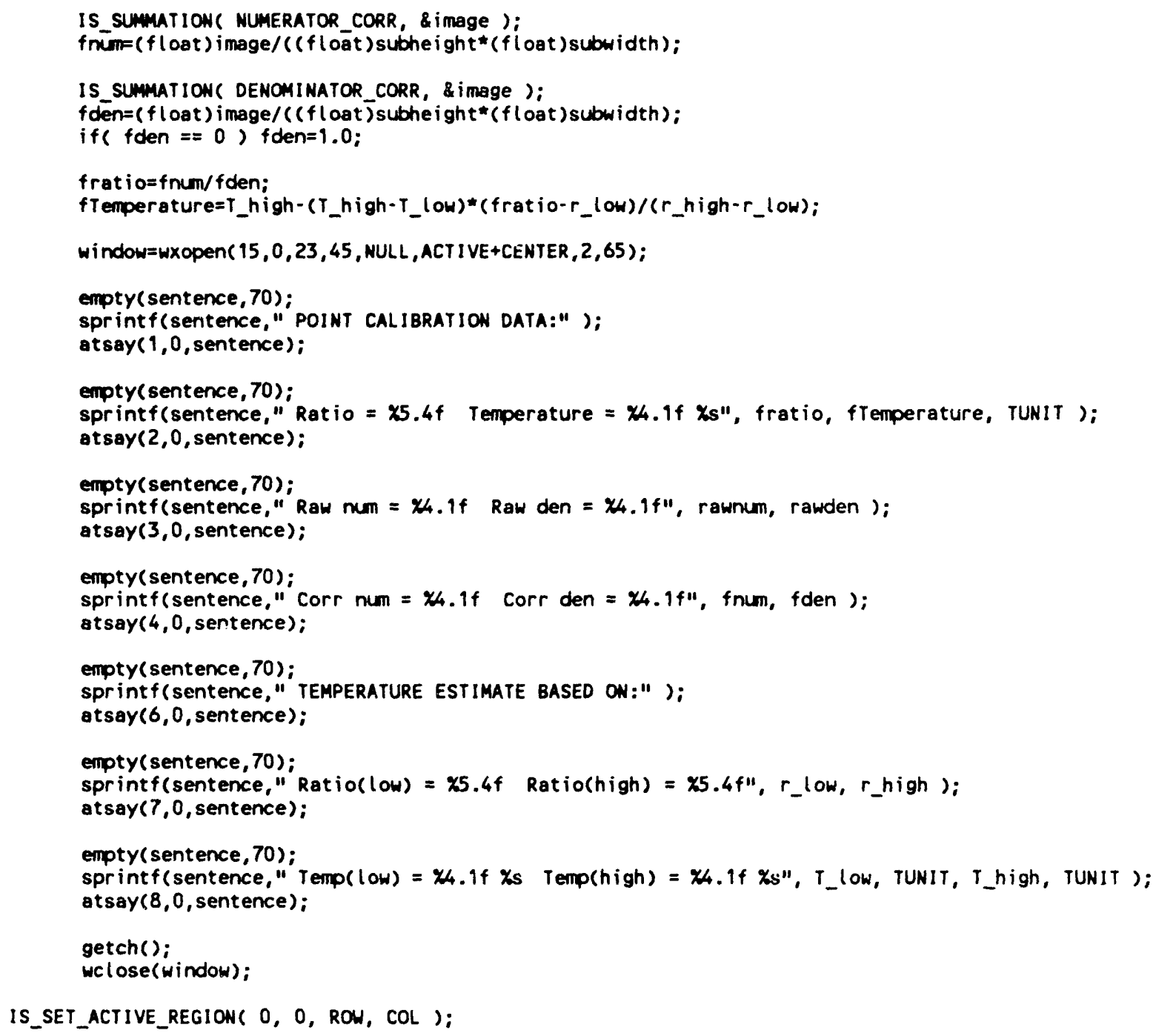


PROGRAM : HEATCOLR.C
DATE : 14 December 1990

PROGRAMAER : K. H. TObin

ROUTINES :

COUNT mZOOMPan ( VCMENU *mouptr)

COWT mZoomPanReset ( VCMENU "muptr)

COUNT MMOnochrome( VCMENU *muptr)

COUNT mColorBar( VCMENU *mouptr )

COUNT mColorBand VCMENU *muptr ) void laad_color( void)
J. K. Jordan

Applied Technology Division

Oak Ridge National Laboratory

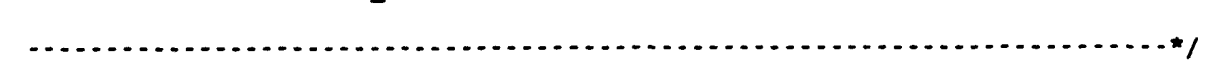

*include "heat.h"

COUNT mZOOMPanReset( VCMENU *muptr )

$c$

Is_zoom_AND_PANC Activebuffer, $1,0,0$ );

return(ō);

3

COUNT mZOOMPan ( VCMENU *muptr)

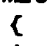

int window;

char sentence [70]:

int cursor_row $=0$, cursor_col =0, zoom $=1$,

c;

empty (sentence, 70);

window $=$ wxopen $(19,0,23,45$, NULL, ACTIVE+CENTER , 0,0);

atsay $(0,0, "$ Zoom with PguP and PgDN keys, ");

atsay $(1,0, "$ Pan/scroll with arrow keys ..." ");

while( $(c=\operatorname{getch}()) !=$ DTCR )

if

if $c==0) c=\operatorname{getch}()$;

if ( $c==0$ TUP ) cursor_row=cursor_row 4 ;

if ( $c==0$ TDOWN ) cursor_row=cursor_row-4;

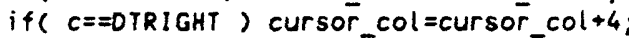

if $(c==0$ TLEFT) cursor_col=cursor_col-4;

if $\left(c==\right.$ DTP gUP ) 200n $200 \mathrm{~m}^{\star 2}$;

if $(c==0$ TPGON ) zoomezoom/2;

if ( cursor_col>COL-1) cursor_col $=0$;

if ( cursor $\mathrm{col}<0$ ) cursor $\mathrm{COl}=\mathrm{COL}-1$;

if ( cursor_row>ROW-1) cursor_rOw=0;

if ( cursor_row<0) cursor_rOW=ROW-1;

if $(200 m>8) 200 m=8$;

if( zoome1) zoome1;

IS_ZOOM_AND_PAN(ACt iveBuffer,zoom, cursor_rOW, cursor_COl); 


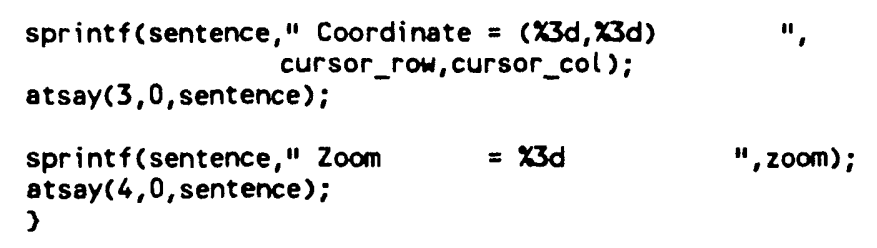

IS_SET_ACTIVE_REGION( OVLY_ROW, OVLY_COL, OVLY_HEIGHT, OVLY_HIDTH ); IS_COPY,REGION ( ACt iveBuffēr, OVLY_BUF, OVLY_ROW, OVLY_COL ); switch_display ( OVLY_BUf );

ACt i veOLUT=COLOR_LUT;

update_status_box();

empty(sentence, 70$)$;

window=wxopen $(19,0,23,45$, NULL, ACT IVE+CENTER , 2,65);

atsay $(0,0$," Select [R] ed, [G] reen, [B] lue color bar");

atsay $(1,0, "$ UP/DOWN arrow keys to shift color bar");

atsay (2,0," RIGHT/LEFT arrow keys to broaden color bar"); atsay $(3,0, "$ ENTER key to select color scheme");

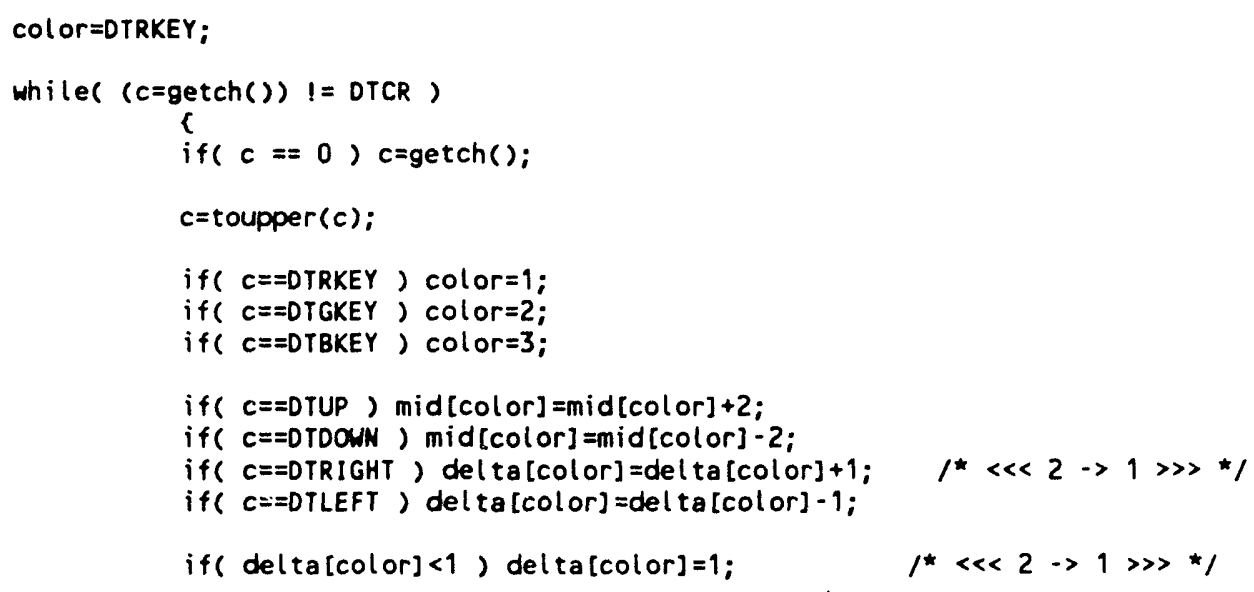


if( delta[color]>(low [color-1]-high [colort1])/2-1)

del ta $[$ color $]=($ low $[$ color -1$]-h i g h[$ colort 1$]) / 2-1$;

if( $\operatorname{mid}[$ color] +del ta [color] $>$ low [color -1$])$ mid [color] $=\operatorname{mid}[$ color] -2 ;

if ( mid[color] -del ta [color] <high [colort1]) mid [color] =mid[color]+2;

high [color] =mid [color]+del ta [color]:

low [color] =mid [color] - del ta [color];

for $(i=0 ; i<$ GREY; $i++)<$ rgb_lut $[1][i]=i$;

rgb_lut [2] $[i]=i$;

rgb_lut [3] [i] =i ; )

fork $i=\operatorname{low}[1] ; i<h i g h[1] ; i++)($ rgb_lut [1] $[i]=$ GREY-1;

rgb_lut [2] [i] $=0$;

rgb_lut [3] $[i]=0$;

for $(i=$ low [2]; i<high[2]; $i++)($ rgb_lut [2] [i]=GREY-1;

rgb_lut $[1][i]=0$;

rgb_lut [3] $[i]=0$;

)

for $i=\operatorname{low}[3] ; i<h i g h[3] ; i++)<$ rgb_lut [3] $[i]=$ GREY-1;

rgb_lut [2] $[i]=0$;

rgb_lut [1] $[i]=0$;

IS LOAD OLUT (ACT IVEOLUT, rgb lut [1], rgb lut [2], rgb lut [3]); IS_SELECT_OLUT (ACTIVEOLUT);

welose(window);

IS SET ACTIVE REGION( 0,0 , ROW, COL );

switch_displays( ActiveBuffer);

return(0);

了

/t

int red[GREY], green [GREY], blue [GREY];

COUNT mColorBand( VCMENU *muptr )

int $i, j, c$;

static int mid=128, del ta $=20$;

int high, low:

int window;

char sentence [70]:

ACt $\mathrm{i}$ VEOLUT $=$ COLOR_LUT;

update status box();

1: GIT ACIIVE REGION( OVLY_ROW, OVLY_COL, OVLY_HEIGHT, OVLY WIDTH);

is CIIIY REGIOÄ́ Act iveBuffër, OVLY_BÜF, OVLY_RÖW, OVLY COI $)_{i}$

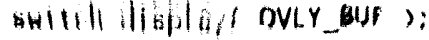

empirisenterice, 10$)$; 


\section{A-48}

window=wXopen $(20,0,23,45$, NULL, ACT I VE+CENTER , 2, 65);

atsay $(0,0, "$ UP/DOWN arrow keys to shift color band");

atsay $11,0, "$ RIGHT/LEFT arrow keys to broaden color band");

atsay (2,0," ENTER key to select color scheme");

while( $(c=\operatorname{getch}()) !=$ DTCR $)$

iff $c==0) c=\operatorname{getch}()$;

if( $c==$ DTUP ) mid=mid+2;

if( $c==$ DTDON ) mid=mid -2 ;

if $c==$ DTRIGHT) del t $a=d e(t a+2$;

if $(c==$ DTLEFT ) del ta=del ta-2;

if( del ta<2) del ta=2;

if ( del ta>(GREY/2)-1) del ta=(GREY/2)-1;

if ( mid+del ta>GREY-1) mid=mid-2;

if( mid-del $t a<0)$ mid=mid+2;

\section{high=midtdel to;}

low=mid-del ta;

for $(i=0 ; i<$ low; $i++)(\operatorname{red}[i]=i ; \operatorname{green}[i]=i ; b l u e[i]=i ;)$

for $(i=h i g h ; i<G R E Y ; i++)(\operatorname{red}[i]=i ; \operatorname{green}[i]=i ;$ blue $[i]=i$;

for $i=$ (ow; $i<h i g h ; i++)$

red $[i]=$ red_lut $[($ int $)($ GREY* $((f$ loat $) i-$ low $) /(f$ loat $)($ high-low $))]$; green $[i]=g r e e n \_l u t\left[(\right.$ int $)\left(\operatorname{GREY}^{\star}((f\right.$ loat $) i-$ low $) /($ float $)($ high-low $\left.\left.)\right)\right]$; blue $[i]=b$ lue_lut $[($ int $)(\operatorname{GREY} *((f$ loat $) i-$ low $) /($ float $)($ high-low $))]$;

IS LOAD OLUT (ACt iveOLUT, red, green, blue):

IS_SELECT_OLUT (ACTIVEOLUT);

wclose(window);

IS_SET_ACTIVE_REGION( 0,0, ROW, COL ); switch_displays ActiveBuffer);

return(0):

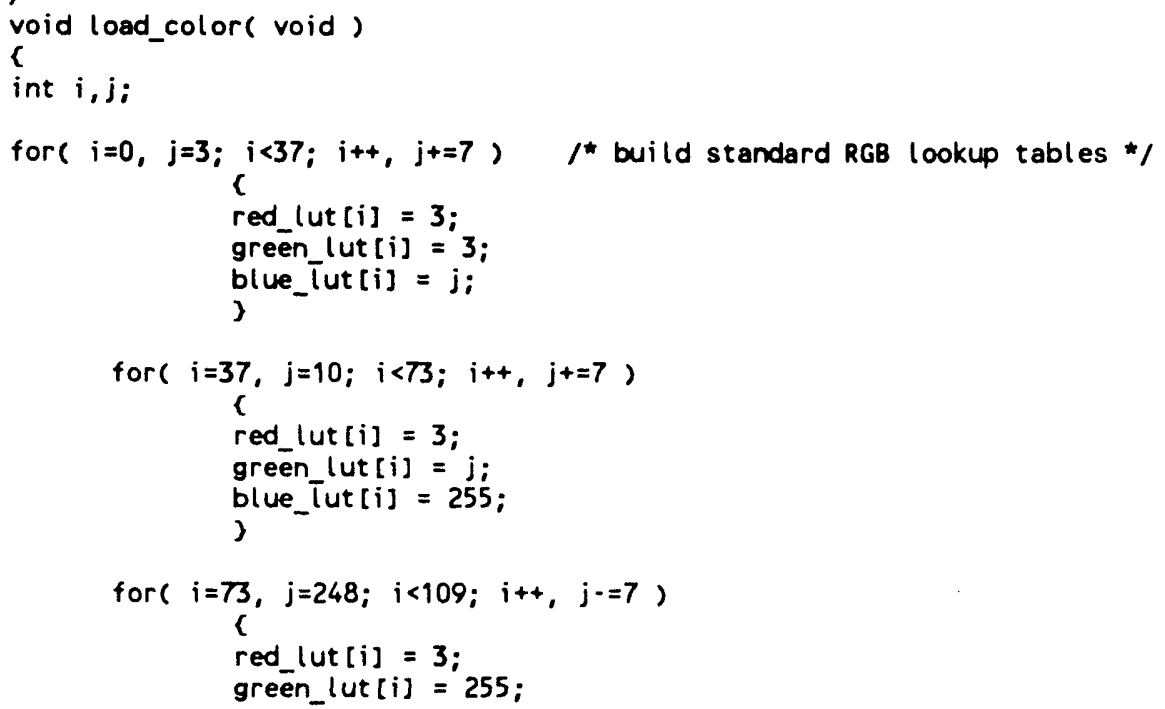




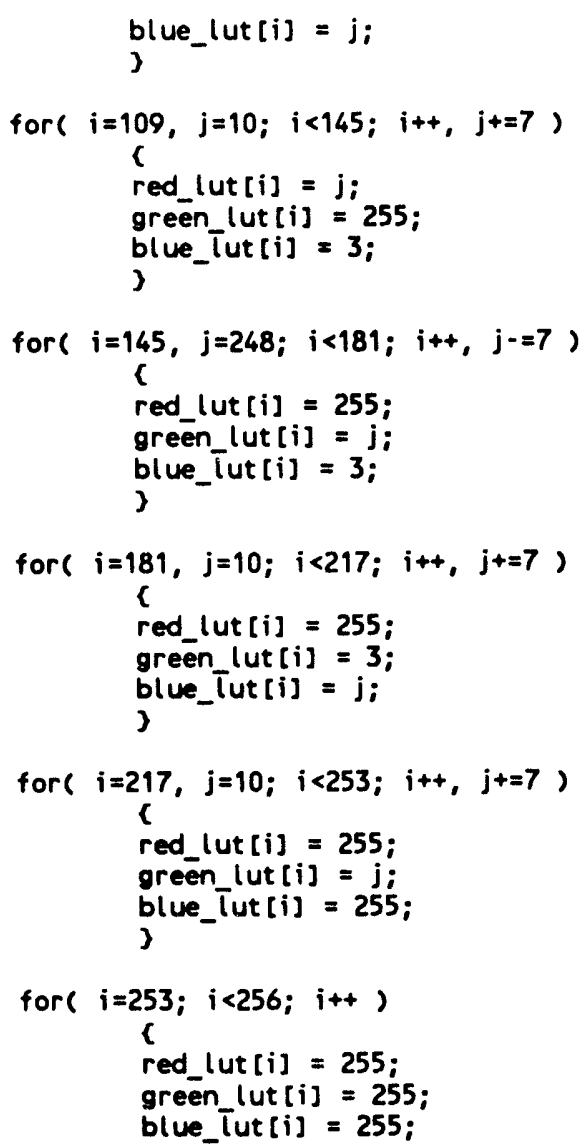




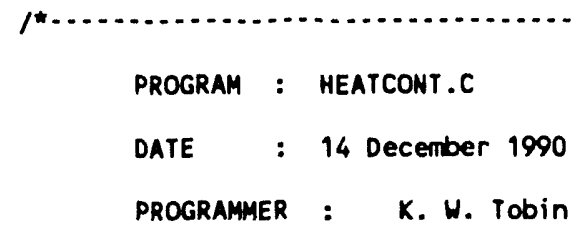

ROUTINES :
J. K. Jordan

Applied Technology Division Oak Ridge National Laboratory

COUNT mContrastLinear ( VCMENU "mnuptr )

COUNT mContrastEqual ( VCMENU imnuptr)

COUNT mSelectModLUT( VCMENU "mnuptr )

COUNT mSelectDefLUT( VCMENU *mnuptr )

\#include "heat.h"

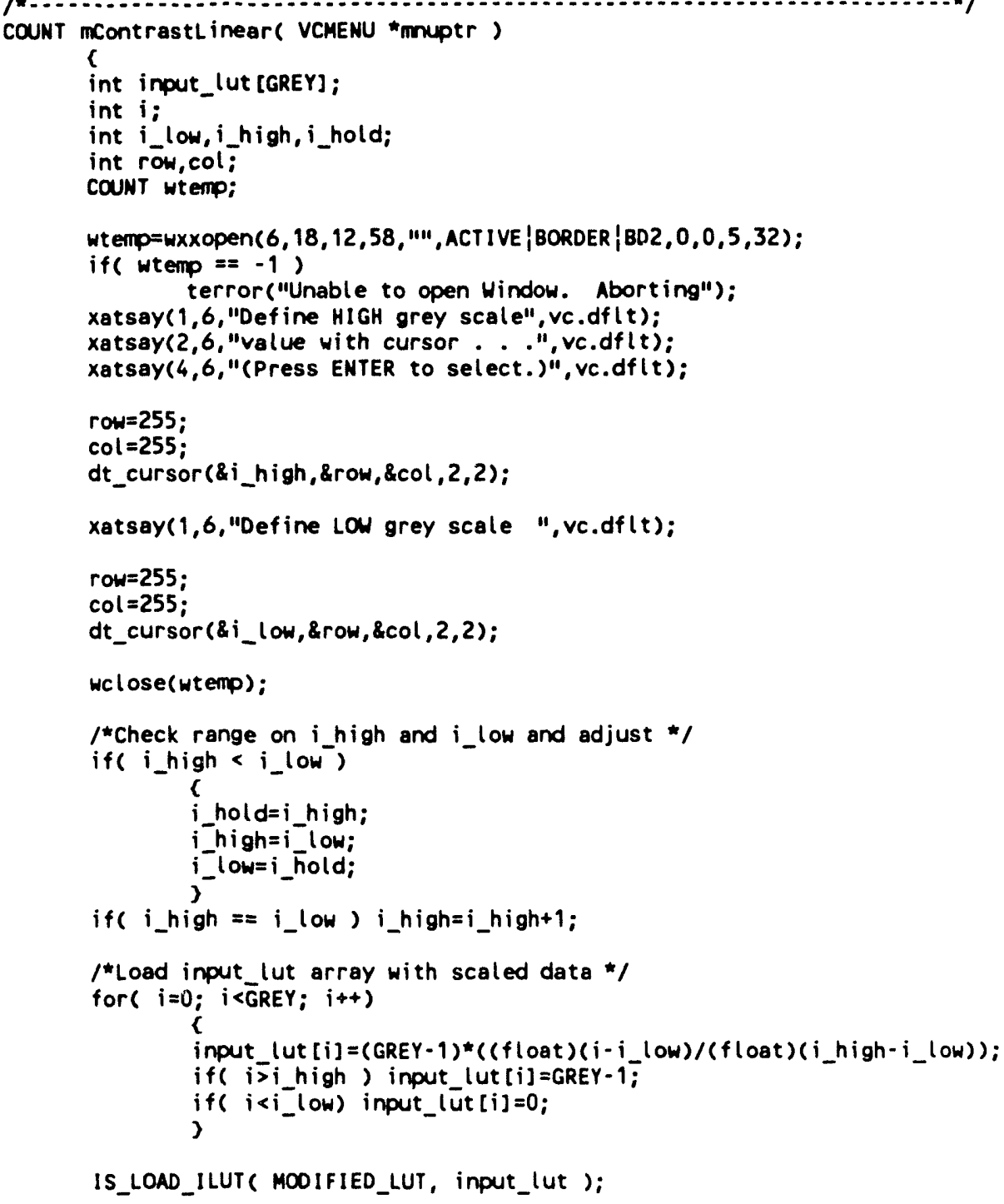


IS_PERFORM_FEEDBACK( ACtiveBuffer, MOOIFIED_LUT, ACtiveBuffer );

return $(0)$ :

3

long pdf [GREY];

floot cdf [GREY];

COUNT mContrastequal( VCMENU *mouptr)

r

int input_lut [GREY];

int $i, j$;

floot sum, den;

1* Lood pdf vector \#/

Is_hISTOGRAM( ActiveBuffer, pdf);

podf $[0]=0$;

sum=0.0;

den=( fl oat)ROW* (float)COL;

1* Sum number of pdf pixels *I

1* (This also works) *I

t* den=0.0;

for $i=0$; $i<$ GREY; $i++$ )

$\star /$

dent $=(f$ loot $)$ pdf $[i]$;

1 *alculate cof vectors */

for ( $i=0$; $i<$ GREY; $i++$ )

sumt $=$ (float)pdf [i] ; cdf $[i]=$ sum $/$ den;

)

1* Calculate lut vector. */

for $(i=0$; $i<G R E Y ; i++)$

$c$

input_lut $[i]=(\text { GREY }-1)^{\star} \operatorname{cdf}[i]$;

$$
\text { ) }
$$

IS_LOND_ILUT( MOOIFIED_LUT, input_lut );

IS_PERFORM_FEEDBACK( ACtiveBuffer, MODIFIED_LUT, ActiveBuffer );

return(0):

)

COUNT mSelectModLUT( VCMENU *moptr)

c

ACt iveI LUT = MOO I FIED LUT ;

IS_SELECT_ILUT( ACT IVEILUT);

update_stätus_box( );

return $(0)$;

了

COUNT mSelectDefLUT( VCMENU *mnuptr )

c

Act $i$ VeILUT =DEFAULT_LUT:

IS_SELECT_ILUT( ACETiVeILUT);

update_stātus_box():

return $(0)$;

) 
Internal Distribution

1-5. D. J. Adams

6. D. L. Beshears

7. M. R. Cates

8. A. Clemons

9. W. S. Key

10-14. K. W. Tobin

15. D. A. Waters

16. Applied Technology Library

17. Central Research Library

18. Y-12 Patent Section

19. Y-12 Technical Library

20-21. Laboratory Records Department

22. Laboratory Records-RC

External Distribution

23-25. C. D. MacArthur, WL/POTC, Wright-Patterson Air Force Base, Ohio 45433-6563

26. C. G. Murawski, WL/POTC, Wright-Patterson Air Force Base, Ohio 45433-6563

27. B. W. Noel, Los Alamos National Laboratory, MEE-5 Division, Los Alamos, New Mexico 87545

28. D. Turley, EG\&G Energy Measurements, Inc., 130 Robin Hill Road, Goleta, California 93117

29-38. Office of Scientific and Technical Information, P.O. Box 62, Oak Ridge, Tennessee 37831

Departinent of Energy, Oak Ridge Field Office

39. Assistant Manager, Energy Research and Development, P.O. Box 2001, Oak Ridge, Tennessee 37831 

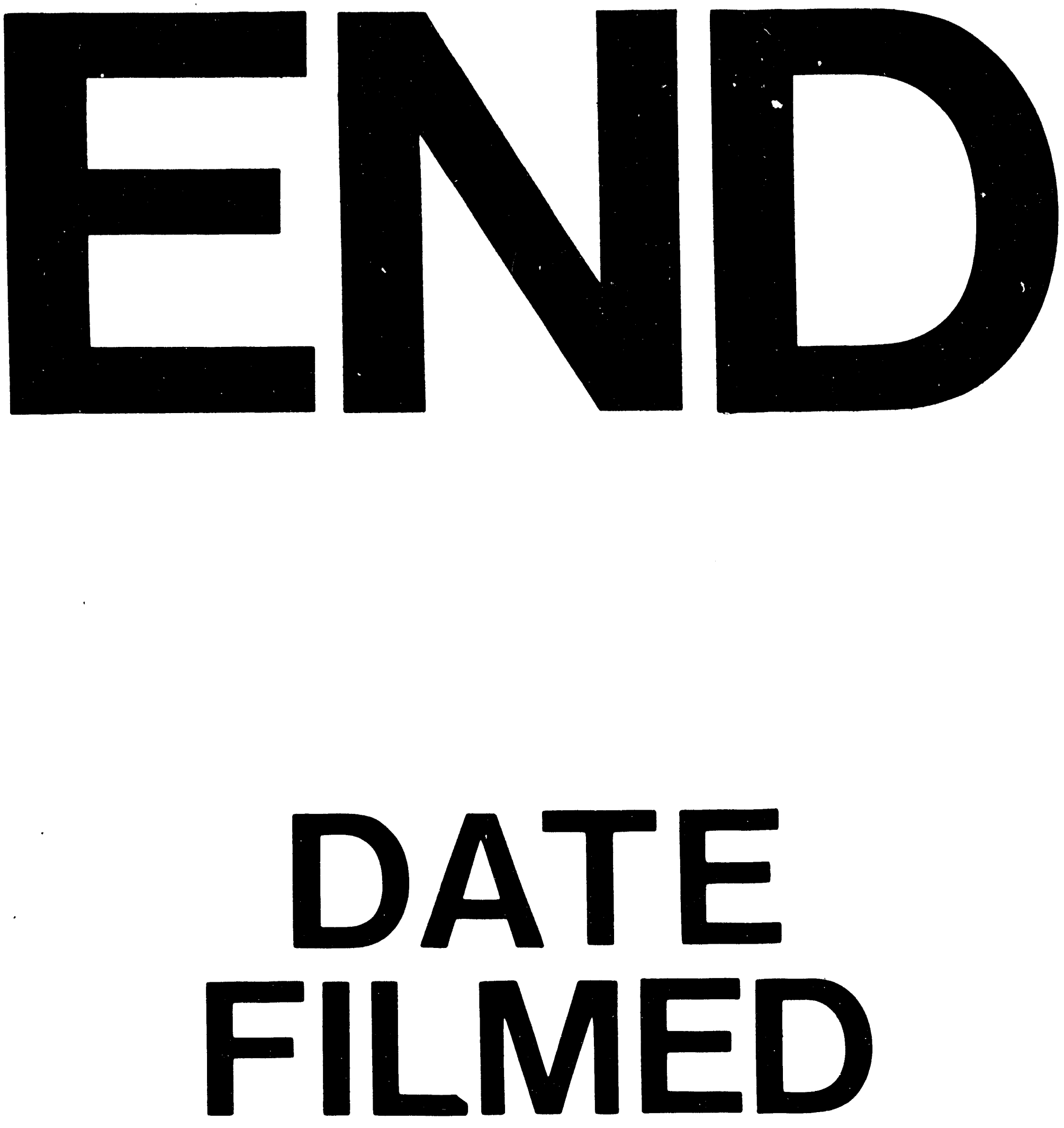

I

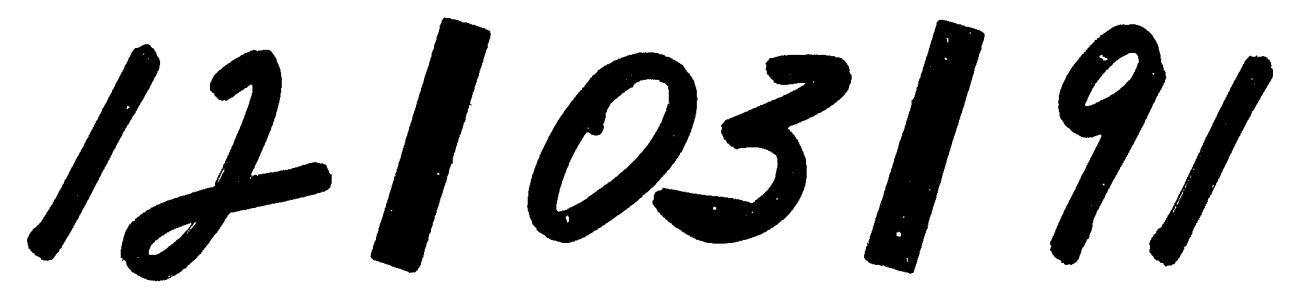

